\title{
Influence of organic compound functionality on aerosol hygroscopicity: dicarboxylic acids, alkyl-substituents, sugars and amino acids
}

\author{
Aleksandra Marsh et al. \\ Correspondence to: Jonathan P. Reid (j.p.reid@bristol.ac.uk)
}

The copyright of individual parts of the supplement might differ from the CC-BY 3.0 licence. 
Table S0 Parameters required for thermodynamic model predictions (* available from a predefined list). And contents of supplement by page and $S$ number.

\begin{tabular}{|c|c|c|c|}
\hline Compound & $\begin{array}{c}\text { Molar Mass / } \\
\text { g.mol }{ }^{-1} \\
\end{array}$ & UNIFAC Structure & Page and S No \\
\hline DL- Alanine & 89.09 & $\mathrm{CH}_{3} \mathrm{COOH} \mathrm{CHNH} 2$ & P3 S1 \\
\hline L-Asparagine & 132.12 & $\mathrm{COOH} \mathrm{CH} \mathrm{CONH}_{2}$ & P38 S36 \\
\hline L-Aspartic Acid & 133.10 & $\mathrm{CH}_{2}(\mathrm{COOH})_{2} \mathrm{CHNH}_{2}$ & P37 $\quad$ S35 \\
\hline L-Arginine & 174.2 & - & $\mathrm{P} 4 \quad \mathrm{~S} 2$ \\
\hline Glycine & 75.06 & $\mathrm{COOH} \mathrm{CHNH} 2$ & P5 S3 \\
\hline L-Histidine & 155.15 & - & P6 S4 \\
\hline L-Lysine & 146.19 & $\mathrm{COOH} \mathrm{CHNH}{ }_{2}\left(\mathrm{CH}_{2}\right)_{3} \mathrm{CH}_{2} \mathrm{NH}_{2}$ & P7 S5 \\
\hline L-Proline & 115.13 & - & P8 S6 \\
\hline L-Threonine & 119.12 & $\mathrm{OH} \mathrm{CH}_{3} \mathrm{CH} \mathrm{COOH} \mathrm{CHNH} 2$ & P9 S7 \\
\hline L-Valine & 117.15 & $\left(\mathrm{CH}_{3}\right)_{2} \mathrm{CH} \mathrm{COOH} \mathrm{CHNH}{ }_{2}$ & $\mathrm{P} 10 \quad \mathrm{~S} 8$ \\
\hline Oxalic Acid* & 90.03 & $(\mathrm{COOH})_{2}$ & $\begin{array}{ll}\text { P13 } & \text { S11 } \\
\end{array}$ \\
\hline Malonic Acid* & 104.062 & $(\mathrm{COOH})_{2} \mathrm{CH}_{2}$ & $\mathrm{P} 14 \quad \mathrm{~S} 12$ \\
\hline Succinic Acid* & \multirow{2}{*}{118.09} & $(\mathrm{COOH})_{2}\left(\mathrm{CH}_{2}\right)_{2}$ & P15 S13 \\
\hline Methyl Malonic acid & & $\left(\mathrm{CH}_{3}\right)(\mathrm{CH})(\mathrm{COOH})_{2}$ & P19 $\quad \mathrm{S} 17$ \\
\hline Glutaric Acid* & \multirow{3}{*}{132.116} & $(\mathrm{COOH})_{2}\left(\mathrm{CH}_{2}\right)_{3}$ & P16 $\mathrm{S} 14$ \\
\hline Methyl Succinic Acid* & & $\left(\mathrm{CH}_{3}\right)\left(\mathrm{CH}_{2}\right)(\mathrm{CH})(\mathrm{COOH})_{2}$ & P20 $\quad \mathrm{S} 18$ \\
\hline Dimethyl Malonic Acid & & $\left(\mathrm{CH}_{3}\right)_{2}(\mathrm{C})(\mathrm{COOH})_{2}$ & P36 $\quad$ S34 \\
\hline Adipic Acid* & \multirow{5}{*}{146.14} & $(\mathrm{COOH})_{2}\left(\mathrm{CH}_{2}\right)_{4}$ & P17 $\mathrm{S} 15$ \\
\hline 2-Methyl Glutaric Acid* & & $\left(\mathrm{CH}_{3}\right)\left(\mathrm{CH}_{2}\right)_{2}(\mathrm{CH})(\mathrm{COOH})_{2}$ & $\mathrm{P} 24 \quad \mathrm{~S} 22$ \\
\hline 3-Methyl Glutaric Acid* & & $\left(\mathrm{CH}_{3}\right)\left(\mathrm{CH}_{2}\right)_{2}(\mathrm{CH})(\mathrm{COOH})_{2}$ & P26 S24 \\
\hline 2,2-Dimethyl Succinic Acid* & & $\left(\mathrm{CH}_{3}\right)_{2}\left(\mathrm{CH}_{2}\right)(\mathrm{C})(\mathrm{COOH})_{2}$ & P23 $\quad$ S21 \\
\hline 2,3-Dimethyl Succinic acid & & $\left(\mathrm{CH}_{3}\right)_{2}(\mathrm{CH})_{2}(\mathrm{COOH})_{2}$ & P35 $\quad$ S33 \\
\hline Pimelic Acid & \multirow{5}{*}{160.17} & $(\mathrm{COOH})_{2}\left(\mathrm{CH}_{2}\right)_{5}$ & P18 S16 \\
\hline 2,2-Dimethyl Glutaric Acid & & $\left(\mathrm{CH}_{3}\right)_{2}\left(\mathrm{CH}_{2}\right)_{2}(\mathrm{C})(\mathrm{COOH})_{2}$ & P22 $\mathrm{S} 20$ \\
\hline 3-Methyl Adipic Acid & & $(\mathrm{CH} 3)_{1}(\mathrm{CH} 2)_{3}(\mathrm{CH})(\mathrm{COOH})_{2}$ & $\mathrm{P} 25 \quad \mathrm{~S} 23$ \\
\hline 3,3-Dimethyl Glutaric Acid & & $\left(\mathrm{CH}_{3}\right)_{2}\left(\mathrm{CH}_{2}\right)_{2}(\mathrm{C})(\mathrm{COOH})_{2}$ & P27 S25 \\
\hline Diethyl Malonic Acid & & $(\mathrm{CH} 3)_{2}(\mathrm{CH} 2)_{2}(\mathrm{C})(\mathrm{COOH})_{2}$ & P21 $\mathrm{S} 19$ \\
\hline Citric Acid* & 192.12 & $(\mathrm{COOH})_{3}\left(\mathrm{CH}_{2}\right)_{2} \mathrm{C}^{(\mathrm{OH})}$ & P11 S9 \\
\hline Tartaric Acid & 150.09 & $(\mathrm{COOH})_{2}(\mathrm{OH})_{2}(\mathrm{CH})_{2}{ }^{(\mathrm{OH})}$ & $\mathrm{P} 12 \mathrm{~S} 10$ \\
\hline Sorbitol & 182.17 & $\left(\mathrm{CH}_{2}{ }^{\text {[alc] }}\right)_{2}-\left(\mathrm{CH}_{1}{ }^{(\mathrm{OH})}\right)_{4}(\mathrm{OH})_{6}$ & P31 $\mathrm{S} 29$ \\
\hline D-(+)-Trehalose Dihydrate & 378.33 & $(\mathrm{CH})\left(\mathrm{CH}_{2}^{[\mathrm{OH}]}\right)_{8}\left(\mathrm{CHO}^{[\text {ether }]}\right)_{3}(\mathrm{OH})_{8}$ & P32 S30 \\
\hline Galactose & 180.16 & $\mathrm{CHO}\left(\mathrm{CH}_{1}{ }^{(\mathrm{OH})}\right)_{4} \mathrm{CH}_{2}{ }^{(\mathrm{alc})}(\mathrm{OH})_{5}$ & $\begin{array}{ll}\text { P33 } & \text { S31 } \\
\end{array}$ \\
\hline Xylose & 150.13 & $\left(\mathrm{CH}_{2}(\mathrm{OH})\right)_{3} \mathrm{CH}_{2}^{(\text {alc })} \mathrm{CHO}(\mathrm{OH})_{4}$ & P34 S32 \\
\hline PEG4 & 194.23 & 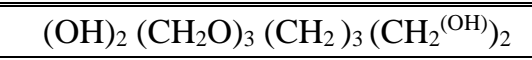 & $\begin{array}{ll}\text { P29 } & \text { S27 } \\
\end{array}$ \\
\hline PEG3 & 150.17 & $(\mathrm{OH})_{2}\left(\mathrm{CH}_{2}\right)_{2}\left(\mathrm{CH}_{2} \mathrm{O}\right)_{2}\left(\mathrm{CH}_{2}{ }^{(\mathrm{OH})}\right)_{2}$ & P28 $\quad$ S26 \\
\hline Erythritol & 122.12 & $\left(\mathrm{CH}^{(\mathrm{OH})}\right)_{2}\left(\mathrm{CH}_{2}{ }^{(\mathrm{OH})}\right)_{2}(\mathrm{OH})_{4}$ & P30 $\quad$ S28 \\
\hline
\end{tabular}

Table S0.1 Fitted parameters for upper and lower MFS vs water activity of compounds in each class, amino and organic acids, sugars and alcohols, as shown in Figure 11b) in the manuscript. The power law coefficient $P$ is used to calculate energy parameter $C$ for the first to $(n-1)$ th layers, hence $C_{i}=(i / n)^{P}$, where $i$ is the layer number and $n$ is the total number of hydration layers, here $n=8$ for all compounds except glycine $(n=3)$ and 2,2-dimethyl glutaric acid $(n=$ 
16). MSE is a normalized mean-square error, equal to $\left(\frac{1}{n_{p}}\right) \sum_{i=1}^{n_{p}}\left(\left(m_{\text {model }, i}-m_{\text {data, } i}\right) /\left(m_{m o d e l, i}\right)\right)^{2}$, where $n_{p}$ is the number of data points.

\begin{tabular}{ccc}
\hline Solute & $\boldsymbol{P}$ & MSE \\
\hline $\begin{array}{c}\text { Amino acid Upper } \\
\text { (Glycine) }\end{array}$ & -1.934 & 0.00321 \\
\hline $\begin{array}{c}\text { Amino acid Lower } \\
\text { (Asparagine) }\end{array}$ & -0.171 & 0.04151 \\
\hline $\begin{array}{c}\text { Organic acid Upper } \\
\text { (Malonic acid) }\end{array}$ & -0.212 & 0.00819 \\
\hline $\begin{array}{c}\text { Organic acid Lower (2,2 } \\
\text { dimethyl glutaric acid) }\end{array}$ & 0.206 & 0.08315 \\
\hline Sugar Upper (Sorbitol) & -0.522 & 0.01025 \\
\hline Sugar Lower (Trehalose) & -0.870 & 0.01687 \\
\hline Alcohol Upper (Erythritol) & -0.238 & 0.01311 \\
\hline Alcohol Lower (PEG4) & -1.180 & 0.16205 \\
\hline
\end{tabular}

Table S0.2 Fitted parameters for nine amino acids. The power law coefficient $P$ is used to calculate energy parameter $C$ for the first to $(n-1)$ th layers, hence $C_{i}=(i / n)^{P}$, where $i$ is the layer number and $n$ is the total number of hydration layers, here $n=8$ for all compounds except glycine $(n=3)$ and threonine $(n=5)$. MSE is a normalized mean-square error, equal to $\left(\frac{1}{n_{p}}\right) \sum_{i=1}^{n_{p}}\left(\left(m_{\text {model }, i}-m_{\text {data }, i}\right) /\left(m_{\text {model }, i}\right)\right)^{2}$, where $n_{p}$ is the number of data points. (Parameter for L-aspartic acid could not be determined due to data range available.)

\begin{tabular}{ccc}
\hline Solute & $\boldsymbol{P}$ & MSE \\
\hline \hline Alanine & -0.356 & 0.00051 \\
\hline Asparagine & -0.171 & 0.04151 \\
\hline Arginine & -0.993 & 0.04039 \\
\hline Glycine & -1.934 & 0.00321 \\
\hline Histidine & -0.502 & 0.02211 \\
\hline Lysine & -1.225 & 0.00667 \\
\hline Proline & -0.619 & 0.03764 \\
\hline Threonine & -0.960 & 0.20107 \\
\hline Valine & -0.892 & 0.00397
\end{tabular}

\section{S1 DL-Alanine Hygroscopicity}


Fig. S1.1: Hygroscopicity of DL-Alanine (Sigma Aldrich, Purity $99 \%$ ) at 293.15 K.

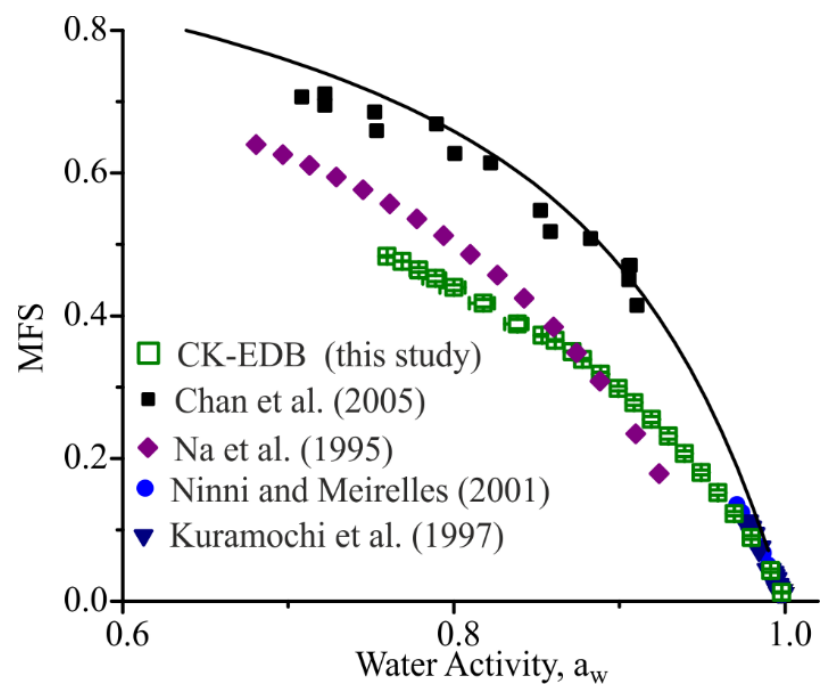

Table S1.1: Pure component refractive index $\left(n_{\text {melt }}\right)$ is determined using molar refraction, assuming ideal mixing for calculation of the melt density $\left(\rho_{\text {melt }}\right)$, from bulk data available in Cai et al. (2016). The variation of density as a function of the root of solute mass fraction $\left(\mathrm{MFS}^{1 / 2}=\mathrm{x}\right)$ is represented by polynomial fit parameters. Upper and lower refer to 95 $\%$ confidence limits for fits to experimental data, (Section 2.2 in manuscript).

\begin{tabular}{c|cc|cccc}
\hline & & & \multicolumn{4}{|c}{ Polynomial fit $\left(\rho_{\text {sol }}=\mathrm{a}+\mathrm{b}_{1} x+\mathrm{b}_{2} x^{2}+\mathrm{b}_{3} x^{3}\right)$} \\
& $n_{\text {melt }}$ & $\rho_{\text {melt }} / \mathrm{g} . \mathrm{cm}^{-3}$ & $\mathrm{a}$ & $\mathrm{b}_{1}$ & $\mathrm{~b}_{2}$ & $\mathrm{~b}_{3}$ \\
\hline \hline Best & 1.6205 & 1.4961 & 999 & 94.14 & -66.93 & 466.48 \\
\hline Upper & 1.6222 & 1.5042 & 999 & 97.38 & -76.61 & 480.88 \\
\hline Lower & 1.6188 & 1.4881 & 999 & 90.98 & -57.61 & 452.44 \\
\hline
\end{tabular}

Table S1.2: Tabulated experimental data points shown in Fig S1.1.

\begin{tabular}{ccc|cc}
\hline $\mathrm{a}_{\mathrm{w}}$ & error $\mathrm{a}_{\mathrm{w}}(+\mathrm{ve})$ & error $\mathrm{a}_{\mathrm{w}}(-\mathrm{ve})$ & MFS & error MFS \\
\hline \hline 0.75966 & 0.00182 & 0.00228 & 0.48336 & $8.66 \mathrm{E}-04$ \\
0.76866 & $1.02 \mathrm{E}-03$ & 0.00128 & 0.47642 & $3.48 \mathrm{E}-04$ \\
0.77876 & 0.00413 & 0.00519 & 0.46428 & 0.00314 \\
0.78887 & 0.00613 & 0.00771 & 0.45228 & 0.00412 \\
0.80001 & 0.00674 & 0.00847 & 0.43959 & 0.00395 \\
0.81774 & 0.00674 & $8.47 \mathrm{E}-03$ & 0.41748 & 0.00512 \\
0.83836 & $6.17 \mathrm{E}-03$ & $7.75 \mathrm{E}-03$ & 0.38848 & $0.39 \mathrm{E}-03$ \\
0.85334 & 0.00473 & $3.00 \mathrm{E}-03$ & 0.37246 & $8.92 \mathrm{E}-04$ \\
0.86108 & 0.00116 & $7.54 \mathrm{E}-04$ & 0.3655 & $5.50 \mathrm{E}-04$ \\
0.87144 & $6.02 \mathrm{E}-04$ & $4.18 \mathrm{E}-04$ & 0.34973 & 0.00165 \\
0.87774 & 0.00139 & $9.81 \mathrm{E}-04$ & 0.3386 & 0.00274 \\
0.88866 & 0.00217 & 0.00153 & 0.31805 & 0.00333 \\
0.89931 & $2.66 \mathrm{E}-03$ & $1.92 \mathrm{E}-03$ & 0.2981 & 0.00381 \\
0.9087 & 0.00257 & 0.00183 & 0.27841 & 0.00426 \\
0.91923 & 0.00256 & 0.00191 & 0.25483 & 0.00416 \\
0.92957 & 0.00248 & 0.00181 & 0.23142 & 0.00392 \\
0.93936 & 0.00243 & 0.00179 & 0.20672 & 0.00361 \\
0.94936 & 0.00206 & $1.54 \mathrm{E}-03$ & 0.18027 & 0.00347 \\
0.9595 & $1.95 \mathrm{E}-03$ & $1.43 \mathrm{E}-03$ & 0.15211 & 0.003 \\
0.96935 & $1.49 \mathrm{E}-03$ & $1.11 \mathrm{E}-03$ & 0.12252 & 0.00254 \\
0.97954 & 0.00127 & $9.28 \mathrm{E}-04$ & 0.08929 & 0.00238 \\
0.99143 & $6.34 \mathrm{E}-04$ & $7.22 \mathrm{E}-04$ & 0.04259 & \\
\hline
\end{tabular}


Fig S2.1: Hygroscopicity of L-Arginine, (Acros Organics, Purity > $98 \%$ ), at 293.15 K. Open squares, these experiments.

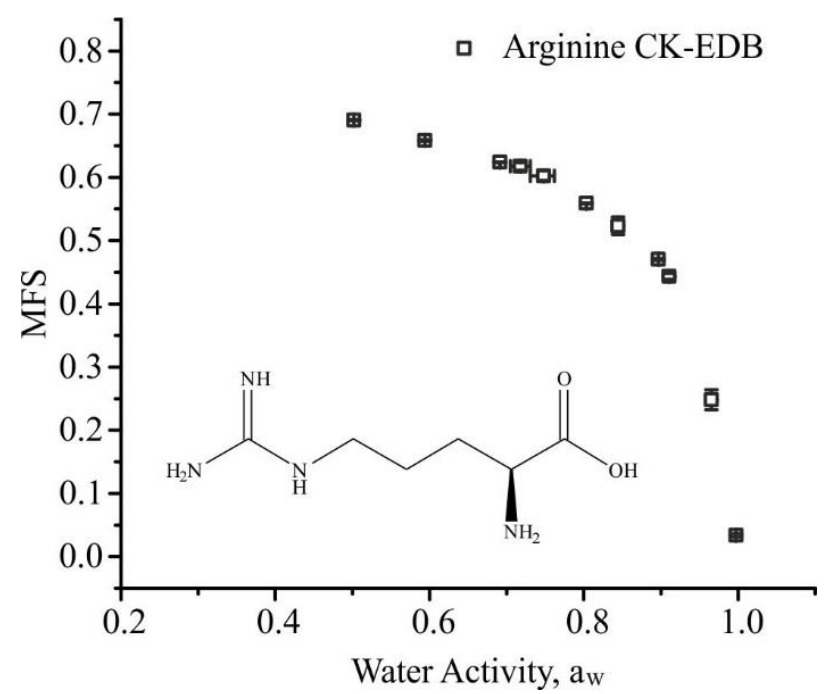

Table S2.1: Pure component refractive index $\left(n_{\text {melt }}\right)$ is determined using molar refraction, assuming ideal mixing for calculation of the melt density $\left(\rho_{\text {melt }}\right)$, from bulk data available in Cai et al. (2016). The variation of density as a function of the root of solute mass fraction (MFS ${ }^{1 / 2}=\mathrm{x}$ ) is represented by polynomial fit parameters. Upper and lower refer to 95 $\%$ confidence limits for fits to experimental data, (Section 2.2 in manuscript).

\begin{tabular}{c|cc|cccc}
\hline & & & \multicolumn{4}{|c}{ Polynomial fit $\left(\rho_{\text {sol }}=\mathrm{a}+\mathrm{b}_{1} \mathrm{x}+\mathrm{b}_{2} \mathrm{x}^{2}+\mathrm{b}_{3} \mathrm{x}^{3}\right)$} \\
& $n_{\text {melt }}$ & $\rho_{\text {melt }} / \mathrm{g.cm} \mathrm{cm}^{-3}$ & $\mathrm{a}$ & \multicolumn{2}{c}{$\mathrm{b}_{1}$} & \multicolumn{2}{c}{$\mathrm{b}_{2}$} & $\mathrm{~b}_{3}$ \\
\hline \hline Best & 1.637 & 1.3995 & 998.6 & 59.85 & 28.54 & 310.48 \\
\hline Upper & 1.6382 & 1.4045 & 998.6 & 61.44 & 24.47 & 317.9 \\
\hline Lower & 1.6358 & 1.3945 & 998.6 & 58.28 & 32.51 & 303.13 \\
\hline
\end{tabular}

Table S2.2: Tabulated experimental data points shown in Fig S2.1.

\begin{tabular}{ccc|cc}
\hline $\mathrm{a}_{\mathrm{w}}$ & error $\mathrm{a}_{\mathrm{w}}(+\mathrm{ve})$ & error $\mathrm{a}_{\mathrm{w}}(-\mathrm{ve})$ & MFS & error MFS \\
\hline \hline 0.50205 & 0.00177 & 0.0021 & 0.69041 & 0.00113 \\
0.59399 & 0.00171 & 0.00206 & 0.65822 & 0.00115 \\
0.69132 & 0.00127 & 0.00157 & 0.62391 & $1.74 \mathrm{E}-04$ \\
0.71788 & 0.01297 & 0.01296 & 0.61768 & 0.00607 \\
0.74796 & 0.0139 & 0.01716 & 0.6026 & 0.00755 \\
0.80315 & $7.87 \mathrm{E}-04$ & 0.001 & 0.55889 & $4.50 \mathrm{E}-04$ \\
0.84439 & 0.00739 & 0.00741 & 0.52351 & 0.014 \\
0.89694 & 0.00128 & 0.00112 & 0.47038 & 0.00138 \\
0.91074 & 0.00174 & 0.00175 & 0.44361 & 0.00473 \\
0.96538 & 0.00317 & 0.00317 & 0.24814 & 0.01569 \\
0.99761 & $5.53 \mathrm{E}-04$ & $5.28 \mathrm{E}-04$ & 0.03416 & 0.00266 \\
\hline
\end{tabular}


Fig S3.1: Hygroscopicity of Glycine, (Santa Cruz Biotech LTD), at 293.15 K. Solid line standard UNIFAC prediction.

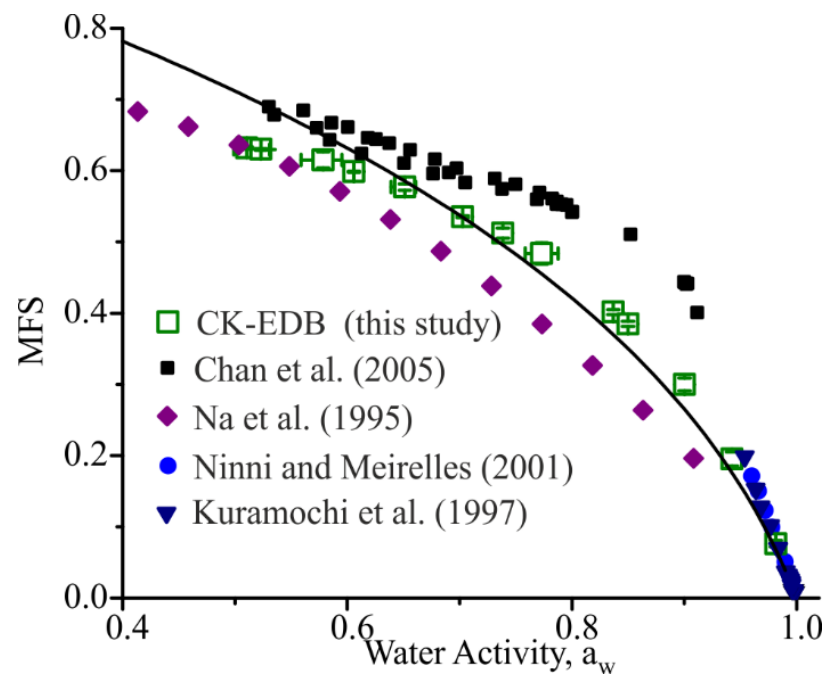

Table S3.1: Pure component refractive index $\left(n_{\text {melt }}\right)$ is determined using molar refraction, assuming ideal mixing for calculation of the melt density $\left(\rho_{\text {melt }}\right)$, from bulk data available in Cai et al. (2016). The variation of density as a function of the root of solute mass fraction (MFS ${ }^{1 / 2}=\mathrm{x}$ ) is represented by polynomial fit parameters. Upper and lower refer to 95 $\%$ confidence limits for fits to experimental data, (Section 2.2 in manuscript).

\begin{tabular}{c|cc|cccc}
\hline & & & \multicolumn{4}{|c}{ Polynomial fit $\left(\rho_{\text {sol }}=\mathrm{a}+\mathrm{b}_{1} \mathrm{x}+\mathrm{b}_{2} \mathrm{x}^{2}+\mathrm{b}_{3} \mathrm{x}^{3}\right)$} \\
& $n_{\text {melt }}$ & $\rho_{\text {melt }} / \mathrm{g.cm} \mathrm{cm}^{-3}$ & $\mathrm{a}$ & $\mathrm{b}_{1}$ & \multicolumn{1}{c}{$\mathrm{b}_{2}$} & $\mathrm{~b}_{3}$ \\
\hline \hline Best & 1.6634 & 1.6905 & 999.47 & 186.75 & -363.66 & 860.4 \\
\hline Upper & 1.6654 & 1.7006 & 999.47 & 192.41 & -382.69 & 883.61 \\
\hline Lower & 1.6613 & 1.6805 & 999.47 & 181.22 & -345.14 & 837.67 \\
\hline
\end{tabular}

Table S3.2: Tabulated experimental data points shown in Fig S3.1.

\begin{tabular}{ccc|cc}
\hline $\mathrm{a}_{\mathrm{w}}$ & error $\mathrm{a}_{\mathrm{w}}(+\mathrm{ve})$ & error $\mathrm{a}_{\mathrm{w}}(-\mathrm{ve})$ & MFS & error MFS \\
\hline \hline 0.51061 & 0.00328 & 0.00389 & 0.63189 & 0.00159 \\
0.52315 & 0.0204 & 0.02421 & 0.62993 & 0.00129 \\
0.57855 & 0.01673 & 0.01985 & 0.61512 & 0.01113 \\
0.60598 & 0.00228 & 0.00276 & 0.59862 & $6.15 \mathrm{E}-04$ \\
0.65105 & 0.00995 & 0.01205 & 0.57691 & 0.00441 \\
0.70256 & 0.00157 & 0.00195 & 0.53551 & 0.00146 \\
0.73844 & 0.0068 & 0.00678 & 0.51233 & 0.00686 \\
0.77309 & 0.01453 & 0.01453 & 0.48382 & 0.01515 \\
0.83663 & 0.0021 & 0.00115 & 0.4015 & 0.00347 \\
0.84998 & 0.00206 & 0.00204 & 0.38496 & 0.00336 \\
0.90029 & 0.00391 & 0.00391 & 0.29984 & 0.00906 \\
0.94266 & 0.00341 & 0.00339 & 0.19519 & 0.00966 \\
0.98152 & 0.00147 & 0.00147 & 0.07624 & 0.00455
\end{tabular}


Fig S4.1: Hygroscopicity of L-Histidine, (VWR Chemicals), open symbols, these CC-EDB experiments.

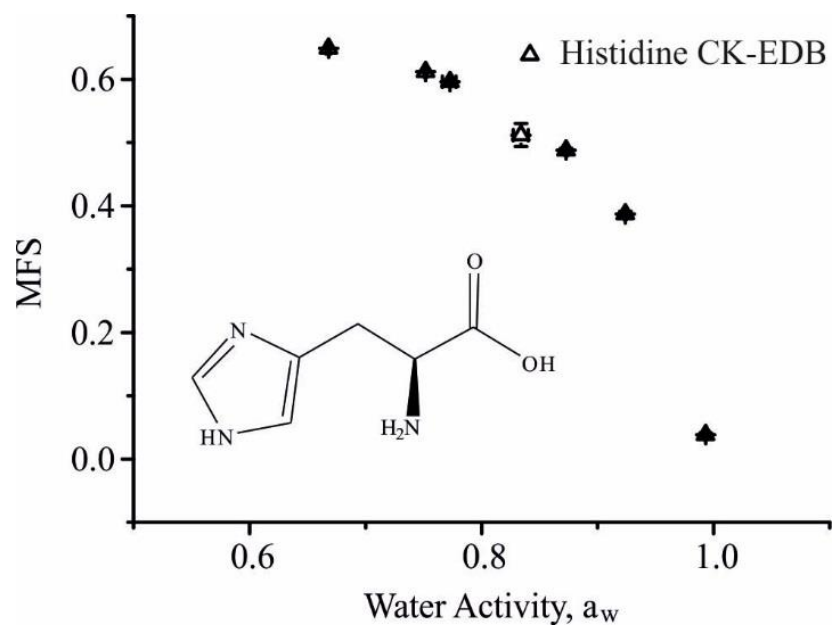

Table S4.1: Pure component refractive index $\left(n_{\text {melt }}\right)$ is determined using molar refraction, assuming ideal mixing for calculation of the melt density $\left(\rho_{\text {melt }}\right)$, from bulk data available in Cai et al. (2016). The variation of density as a function of the root of solute mass fraction (MFS ${ }^{1 / 2}=\mathrm{x}$ ) is represented by polynomial fit parameters. Upper and lower refer to 95 $\%$ confidence limits for fits to experimental data, (Section 2.2 in manuscript).

\begin{tabular}{c|cc|cccc}
\hline & & & \multicolumn{4}{|c}{ Polynomial fit $\left(\rho_{\text {sol }}=\mathrm{a}+\mathrm{b}_{1} \mathrm{x}+\mathrm{b}_{2} \mathrm{x}^{2}+\mathrm{b}_{3} \mathrm{x}^{3}\right)$} \\
& $n_{\text {melt }}$ & $\rho_{\text {melt }}$ g.cm cm $^{-3}$ & $\mathrm{a}$ & $\mathrm{b}_{1}$ & $\mathrm{~b}_{2}$ & $\mathrm{~b}_{3}$ \\
\hline \hline Best & 1.6892 & 1.5378 & 998.9 & 111.5 & -119.61 & 542.86 \\
\hline Upper & 1.6914 & 1.5462 & 998.9 & 115.17 & -130.97 & 558.8 \\
\hline Lower & 1.6871 & 1.5296 & 998.9 & 107.98 & -108.77 & 527.49 \\
\hline
\end{tabular}

Table S4.2: Tabulated experimental data points shown in Fig S4.1.

\begin{tabular}{|c|c|c|c|c|}
\hline$a_{w}$ & error $a_{w}(+v e)$ & error $a_{w}(-v e)$ & MFS & error MFS \\
\hline \multicolumn{5}{|c|}{$293.15 \mathrm{~K}$} \\
\hline 0.66801 & 0.00175 & 0.00214 & 0.64888 & 5.85836E-4 \\
\hline 0.75174 & 0.00105 & 0.00131 & 0.61182 & $3.82887 \mathrm{E}-4$ \\
\hline 0.77265 & 0.00527 & 0.00661 & 0.59614 & 0.00177 \\
\hline 0.83375 & 0.0064 & 0.00643 & 0.51198 & 0.01825 \\
\hline 0.87281 & 0.00111 & 0.00101 & 0.48826 & 0.0027 \\
\hline 0.9239 & $8.9548 \mathrm{E}-4$ & $9.46372 \mathrm{E}-4$ & 0.38721 & 0.00439 \\
\hline 0.99296 & $6.37951 \mathrm{E}-4$ & $6.3374 \mathrm{E}-4$ & 0.03829 & 0.00295 \\
\hline
\end{tabular}




\section{S5 L-Lysine Hygroscopicity}

Fig S5.1: Hygroscopicity of L-Lysine, (Sigma Aldrich, Purity $\geq 98 \%$ ), at 293.15 K. Open squares, these experiments; solid line, UNIFAC model.

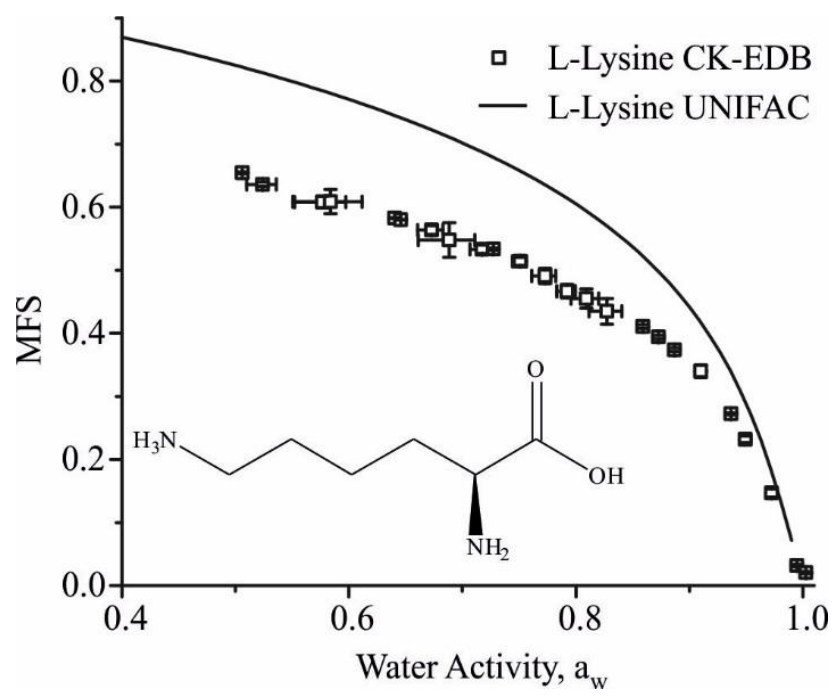

Table S5.1: Pure component refractive index $\left(n_{\text {melt }}\right)$ determined using molar refraction where the melt density ( $\left.\rho_{\text {melt }}\right)$, is determined using a polynomial fit of density to the square root of MFS (MFS ${ }^{1 / 2}=\mathrm{x}$ ). Bulk values used are available in Cai et al. (2016). Upper and lower refer to $95 \%$ confidence limits for fits to experimental data.

\begin{tabular}{c|cc|cccc}
\hline & & & \multicolumn{4}{|c}{ Polynomial fit $\left(\rho_{\text {sol }}=\mathrm{a}+\mathrm{b}_{1} \mathrm{x}+\mathrm{b}_{2} \mathrm{x}^{2}+\mathrm{b}_{3} \mathrm{x}^{3}\right)$} \\
& $n_{\text {melt }}$ & $\rho_{\text {melt }}$ g.cm & $\mathrm{a}$ & $\mathrm{b}_{1} \mathrm{~b}^{-3}$ & $\mathrm{~b}_{2}$ & $\mathrm{~b}_{3}$ \\
\hline \hline Best & 1.5586 & 1.2362 & 998.2 & -15.22 & 309.14 & -56.02 \\
\hline Upper & 1.5614 & 1.2418 & 998.2 & -4.29 & 271.92 & -23.99 \\
\hline Lower & 1.5558 & 1.2306 & 998.2 & -25.93 & 346.35 & -88.05 \\
\hline
\end{tabular}

Table S5.2: Tabulated experimental data points shown in Fig S5.1.

\begin{tabular}{ccc|cc}
\hline $\mathrm{a}_{\mathrm{w}}$ & error $\mathrm{a}_{\mathrm{w}}(+\mathrm{ve})$ & error $\mathrm{a}_{\mathrm{w}}(-\mathrm{ve})$ & MFS & error MFS \\
\hline \hline .50605 & 0.00267 & 0.00316 & 0.65479 & 0.00157 \\
0.52404 & 0.01187 & 0.01406 & 0.63621 & 0.00337 \\
0.57666 & 0.02059 & 0.02439 & 0.60815 & 0.00948 \\
0.58372 & 0.02793 & 0.03308 & 0.60867 & 0.01931 \\
0.64049 & 0.00405 & 0.00494 & 0.58275 & $4.04084 \mathrm{E}-4$ \\
0.64559 & 0.00205 & 0.00251 & 0.57997 & 0.00675 \\
0.67292 & 0.00999 & 0.0122 & 0.56365 & 0.02742 \\
0.68839 & 0.0225 & 0.02735 & 0.54807 & 0.00647 \\
0.71755 & 0.00885 & 0.01092 & 0.53328 & 0.00179 \\
0.72732 & 0.00179 & 0.00223 & 0.53359 & 0.00626 \\
0.75098 & 0.0056 & 0.00696 & 0.51408 & 0.01194 \\
0.77291 & 0.00939 & 0.01164 & 0.49095 & 0.01099 \\
0.79224 & 0.00736 & 0.00909 & 0.46681 & 0.01535 \\
0.80926 & 0.01092 & 0.01352 & 0.45505 & 0.02026 \\
0.82751 & 0.01292 & 0.01604 & 0.43489 & 0.00309 \\
0.85916 & 0.00152 & 0.00197 & 0.41093 & 0.00288 \\
0.87288 & 0.00143 & 0.00143 & 0.39407 & 0.00294 \\
0.88688 & 0.00151 & 0.00151 & 0.3739 & 0.00983 \\
0.90999 & 0.00294 & 0.00337 & 0.33998 & 0.00154 \\
0.93683 & $3.20551 \mathrm{E}-4$ & $3.24824 \mathrm{E}-4$ & 0.27222 & 0.00544 \\
0.94931 & 0.00162 & 0.00162 & 0.23179 & 0.00623 \\
0.97255 & 0.00147 & 0.00147 & 0.14683 & 0.0021 \\
0.99465 & $4.49456 \mathrm{E}-4$ & $4.50168 \mathrm{E}-4$ & 0.03174 & 0.00198 \\
1.00277 & 0.00113 & 0.00149 & 0.02039 &
\end{tabular}




\section{S6 L-Proline Hygroscopicity}

Figure S6.1: Hygroscopicity of L-Proline, (Acros Organics, Purity + $99 \%$ ), at 293.15 K. Open squares, these experiments.

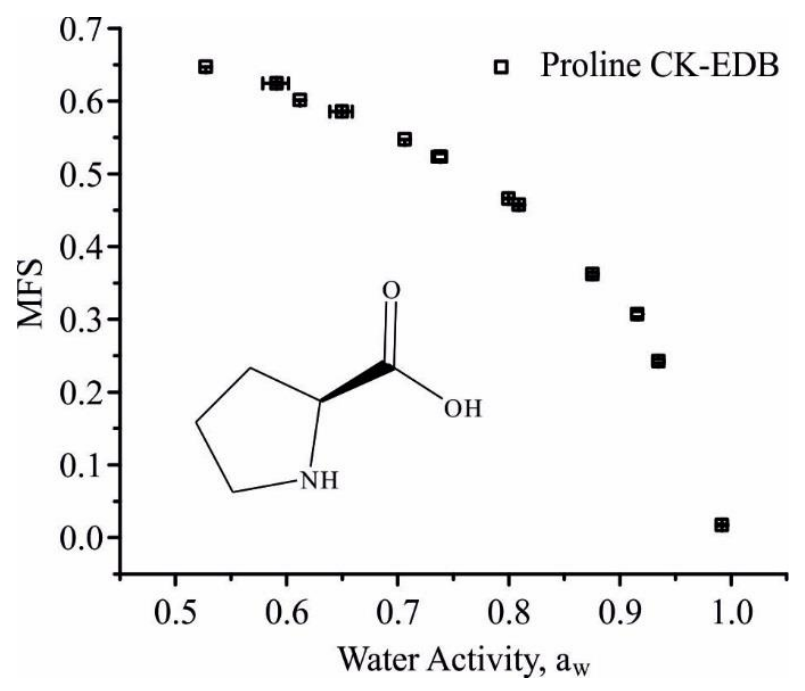

Table S6.1: Pure component refractive index $\left(n_{\text {melt }}\right)$ is determined using molar refraction, assuming ideal mixing for calculation of the melt density $\left(\rho_{\text {melt }}\right)$, from bulk data available in Cai et al. (2016). The variation of density as a function of the root of solute mass fraction (MFS ${ }^{1 / 2}=\mathrm{x}$ ) is represented by polynomial fit parameters. Upper and lower refer to 95 $\%$ confidence limits for fits to experimental data, (Section 2.2 in manuscript).

\begin{tabular}{|c|c|c|c|c|c|c|}
\hline & \multirow[b]{2}{*}{$n_{\text {melt }}$} & \multirow[b]{2}{*}{$\rho_{\text {melt }} /{\mathrm{g} . \mathrm{cm}^{-3}}^{-3}$} & \multicolumn{4}{|c|}{ Polynomial fit $\left(\rho_{\mathrm{sol}}=\mathrm{a}+\mathrm{b}_{1} \mathrm{x}+\mathrm{b}_{2} \mathrm{x}^{2}+\mathrm{b}_{3} \mathrm{x}^{3}\right)$} \\
\hline & & & $\mathrm{a}$ & $\mathrm{b}_{1}$ & $\mathrm{~b}_{2}$ & $\mathrm{~b}_{3}$ \\
\hline Best & 1.5948 & 1.3866 & 999 & 55.7 & 39.01 & 291 \\
\hline Upper & 1.5964 & 1.3945 & 999 & 58.13 & 32.96 & 302.44 \\
\hline Lower & 1.5932 & 1.3788 & 999 & 53.36 & 44.73 & 279.93 \\
\hline
\end{tabular}

Table S6.2: Tabulated experimental data points shown in Fig S6.1.

\begin{tabular}{ccc|cc}
\hline $\mathrm{a}_{\mathrm{w}}$ & error $\mathrm{a}_{\mathrm{w}}(+\mathrm{ve})$ & error $\mathrm{a}_{\mathrm{w}}(-\mathrm{ve})$ & MFS & error MFS \\
\hline \hline 0.52739 & 0.00183 & 0.00218 & 0.647 & $1.53 \mathrm{E}-04$ \\
0.59111 & 0.01061 & 0.01264 & 0.62414 & 0.00218 \\
0.61213 & 0.00154 & 0.00186 & 0.6013 & $1.44 \mathrm{E}-04$ \\
0.64995 & 0.00905 & 0.01098 & 0.58549 & 0.00122 \\
0.70619 & $9.52 \mathrm{E}-04$ & 0.00118 & 0.54716 & $3.08 \mathrm{E}-04$ \\
0.73823 & 0.0057 & 0.00705 & 0.52349 & 0.00436 \\
0.79982 & $7.99 \mathrm{E}-04$ & 0.00101 & 0.46617 & $7.45 \mathrm{E}-04$ \\
0.80883 & 0.00112 & 0.00112 & 0.45742 & $1.45 \mathrm{E}-03$ \\
0.87515 & $1.30 \mathrm{E}-03$ & 0.00103 & 0.36217 & $2.56 \mathrm{E}-03$ \\
0.91551 & 0.00145 & 0.00184 & 0.30701 & 0.00352 \\
0.93455 & $9.07 \mathrm{E}-04$ & $9.29 \mathrm{E}-04$ & 0.24258 & 0.00279 \\
0.99172 & $5.01 \mathrm{E}-04$ & $5.60 \mathrm{E}-04$ & 0.01734 & 0.0011 \\
\hline
\end{tabular}




\section{S7 L-Threonine Hygroscopicity}

Fig S7.1: Hygroscopicity of L-Threonine, (Acros Organics, Purity $98 \%$ ), at 293.15 K. Open squares, these experiments; solid line, UNIFAC model.

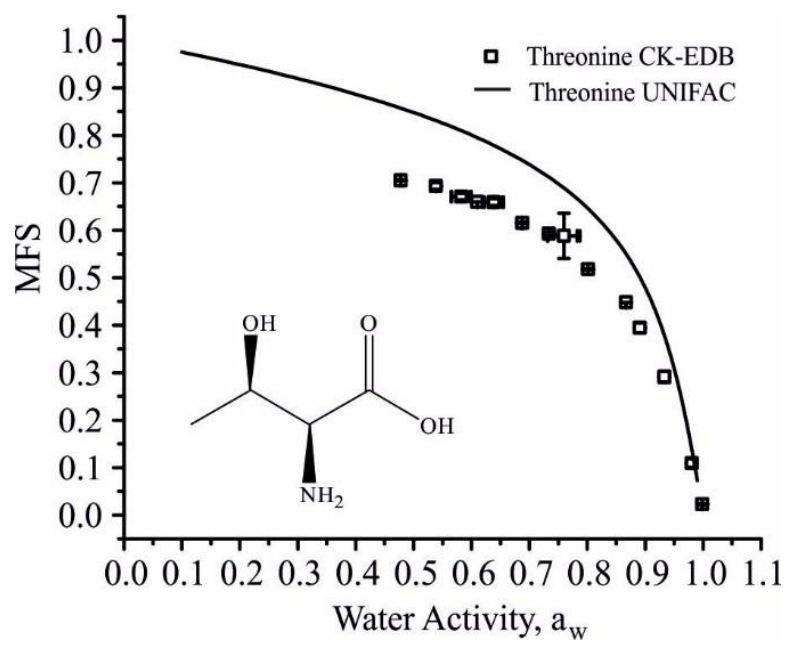

Table S7.1: Pure component refractive index $\left(n_{\text {melt }}\right)$ is determined using molar refraction, assuming ideal mixing for calculation of the melt density $\left(\rho_{\text {melt }}\right.$ ), from bulk data available in Cai et al. (2016). The variation of density as a function of the root of solute mass fraction $\left(\mathrm{MFS}^{1 / 2}=\mathrm{x}\right)$ is represented by polynomial fit parameters. Upper and lower refer to 95 $\%$ confidence limits for fits to experimental data, (Section 2.2 in manuscript).

\begin{tabular}{c|cc|cccc}
\hline & & & \multicolumn{4}{|c}{ Polynomial fit $\left(\rho_{\text {sol }}=\mathrm{a}+\mathrm{b}_{1} \mathrm{x}+\mathrm{b}_{2} \mathrm{x}^{2}+\mathrm{b}_{3} \mathrm{x}^{3}\right)$} \\
& $n_{\text {melt }}$ & $\rho_{\text {melt }}$ g.cm & $\mathrm{a}$ & $\mathrm{b}_{1}$ & $\mathrm{~b}_{2}$ & $\mathrm{~b}_{3}$ \\
\hline \hline Best & 1.6185 & 1.4977 & 999.4 & 94.57 & -68.14 & 468.44 \\
\hline Upper & 1.6274 & 1.5403 & 999.4 & 112.31 & -121.99 & 546.4 \\
\hline Lower & 1.6102 & 1.4575 & 999.4 & 79.24 & -23.63 & 399.69 \\
\hline
\end{tabular}

Table S7.2: Tabulated experimental data points shown in Fig S7.1.

\begin{tabular}{ccc|cc}
\hline $\mathrm{a}_{\mathrm{w}}$ & error $\mathrm{a}_{\mathrm{w}}(+\mathrm{ve})$ & error $\mathrm{a}_{\mathrm{w}}(-\mathrm{ve})$ & MFS & error MFS \\
\hline \hline .47807 & 0.00212 & 0.0025 & 0.70511 & 0.00173 \\
0.53888 & 0.0052 & 0.0062 & 0.69319 & 0.00723 \\
0.58237 & 0.01442 & 0.01711 & 0.67043 & 0.00714 \\
0.60978 & 0.00127 & 0.00154 & 0.65941 & $3.88 \mathrm{E}-04$ \\
0.63867 & 0.01393 & 0.01689 & 0.65875 & 0.00707 \\
0.68779 & 0.00158 & 0.00195 & 0.61529 & 0.00161 \\
0.73352 & 0.0081 & 0.00812 & 0.59255 & 0.0041 \\
0.75945 & 0.02291 & 0.02781 & 0.58815 & 0.04754 \\
0.80118 & 0.00157 & $7.31 \mathrm{E}-04$ & 0.51778 & 0.00135 \\
0.86674 & $7.26 \mathrm{E}-04$ & $4.84 \mathrm{E}-04$ & 0.44784 & $3.66 \mathrm{E}-04$ \\
0.89045 & 0.00426 & 0.00419 & 0.39429 & 0.0104 \\
0.93289 & 0.00438 & 0.00418 & 0.29104 & 0.01212 \\
0.98064 & 0.00213 & 0.00214 & 0.10966 & 0.00862 \\
0.99865 & $7.16 \mathrm{E}-04$ & $4.45 \mathrm{E}-04$ & 0.02317 & 0.00125 \\
\hline
\end{tabular}




\section{S8 L-Valine Hygroscopicity}

Figure S8.1: Hygroscopicity of L-Valine, (Sigma Aldrich, Purity $\geq 98$ \%), at 293.15 K (blue Open symbols, these CKEDB experiments; black filled circles, literature data (Kuramochi et al.); solid black line, UNIFAC model (293.15 K).
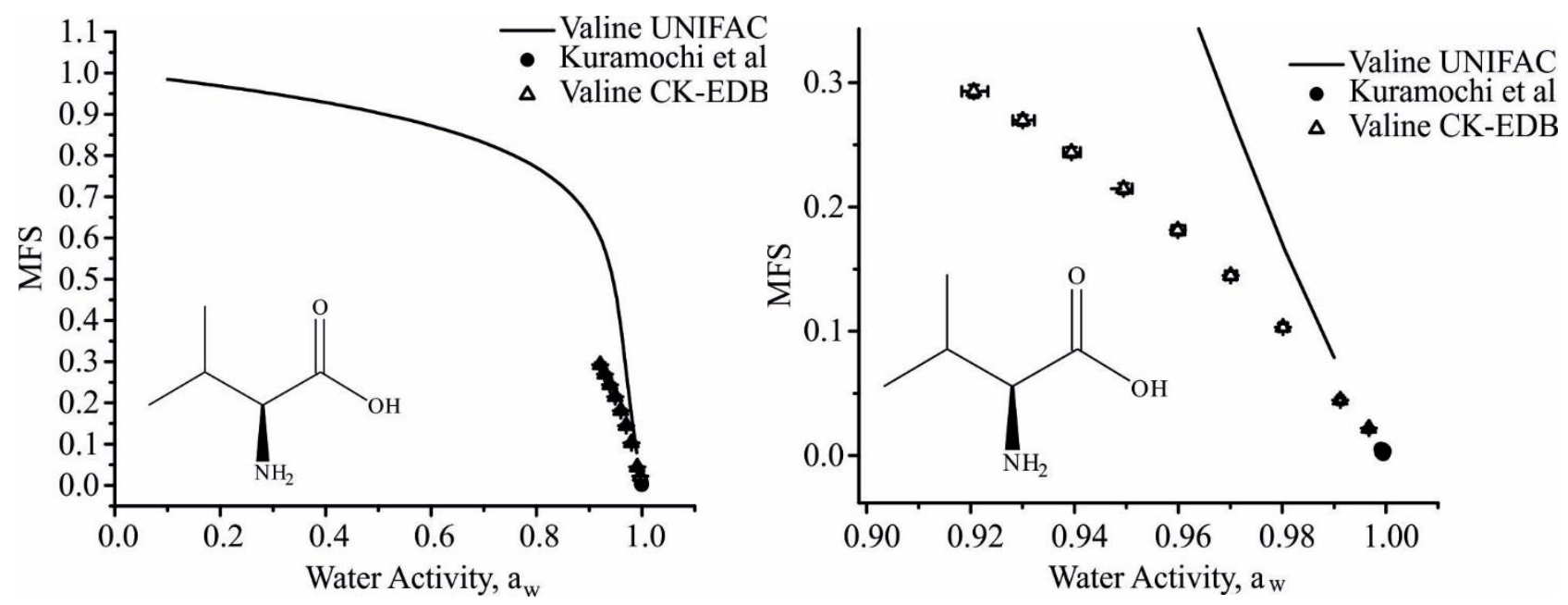

Table S8.1: Pure component refractive index $\left(n_{\text {melt }}\right)$ is determined using molar refraction, assuming ideal mixing for calculation of the melt density $\left(\rho_{\text {melt }}\right)$, from bulk data available in Cai et al. (2016). The variation of density as a function of the root of solute mass fraction $\left(\mathrm{MFS}^{1 / 2}=\mathrm{x}\right.$ ) is represented by polynomial fit parameters. Upper and lower refer to 95 $\%$ confidence limits for fits to experimental data, (Section 2.2 in manuscript).

\begin{tabular}{c|cc|cccc}
\hline & & & \multicolumn{4}{|c}{ Polynomial fit $\left(\rho_{\text {sol }}=\mathrm{a}+\mathrm{b}_{1} \mathrm{x}+\mathrm{b}_{2} \mathrm{x}^{2}+\mathrm{b}_{3} \mathrm{x}^{3}\right)$} \\
& $n_{\text {melt }}$ & $\rho_{\text {melt }}$ g.cm & $\mathrm{a}$ & $\mathrm{b}_{1} \mathrm{~b}^{-3}$ & $\mathrm{~b}_{2}$ & $\mathrm{~b}_{3}$ \\
\hline \hline Best & 1.5791 & 1.2824 & 998.77 & 28.73 & 94.37 & 159.64 \\
\hline Upper & 1.58 & 1.2872 & 998.77 & 29.8 & 92.82 & 164.91 \\
\hline Lower & 1.5781 & 1.2776 & 998.77 & 27.71 & 95.81 & 154.45 \\
\hline
\end{tabular}

Table S8.2: Tabulated experimental data points shown in Fig S8.1.

\begin{tabular}{ccc|cc}
\hline $\mathrm{a}_{\mathrm{w}}$ & error $\mathrm{a}_{\mathrm{w}}(+\mathrm{ve})$ & error $\mathrm{a}_{\mathrm{w}}(-\mathrm{ve})$ & MFS & error MFS \\
\hline \hline \multicolumn{5}{c}{$293.15 \mathrm{~K}$} \\
0.92062 & 0.0027 & 0.00232 & 0.29295 & 0.00499 \\
0.93004 & $2.26 \mathrm{E}-03$ & 0.00195 & 0.26962 & $4.18 \mathrm{E}-03$ \\
0.94941 & 0.00173 & 0.00148 & 0.24396 & 0.00404 \\
0.9599 & 0.00169 & 0.00145 & 0.21478 & 0.00403 \\
0.97008 & 0.00138 & 0.00118 & 0.18125 & 0.00388 \\
0.98014 & 0.00118 & 0.00101 & 0.14482 & 0.00345 \\
0.99117 & $8.66 \mathrm{E}-04$ & $7.41 \mathrm{E}-04$ & 0.10314 & $2.94 \mathrm{E}-03$ \\
0.99669 & $5.34 \mathrm{E}-04$ & $5.69 \mathrm{E}-04$ & 0.04451 & 0.00201 \\
\end{tabular}




\section{S9 Citric Acid Hygroscopicity}

Figure S9.1: Hygroscopicity of Citric Acid, (Sigma Aldrich, Purity $99 \%$ ), at 293.15 K. Open squares, these EDB experiments; solid line, UNIFAC model.

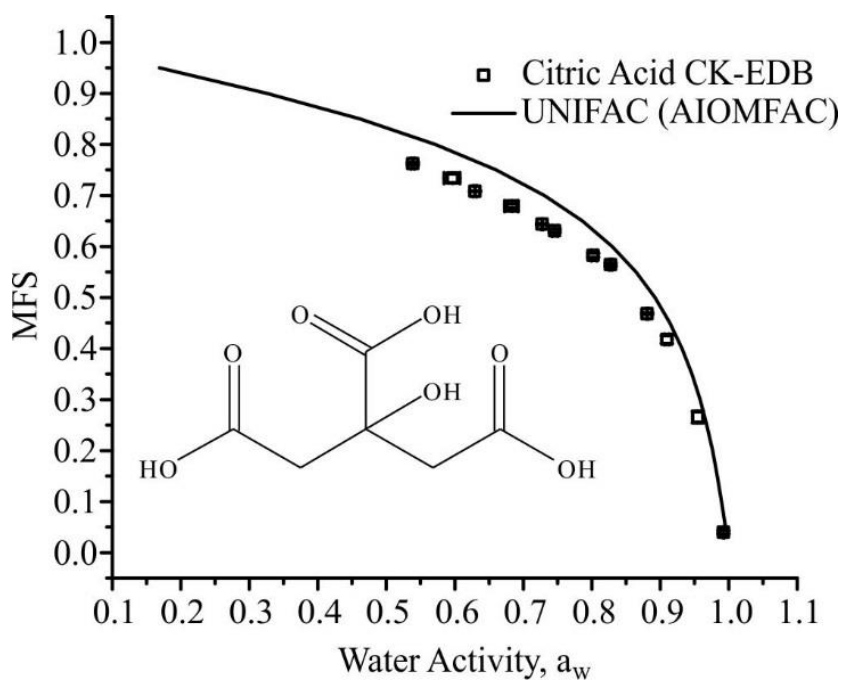

Table S9.1: Pure component refractive index $\left(n_{\text {melt }}\right)$ determined using molar refraction where the melt density $\left(\rho_{\text {melt }}\right)$ is determined using a polynomial fit of density to the square root of MFS $\left(\mathrm{MFS}^{1 / 2}=\mathrm{x}\right)$. Bulk values used are available in Cai et al. (2016). Upper and lower refer to $95 \%$ confidence limits for fits to experimental data.

\begin{tabular}{c|cc|cccc}
\hline & & & \multicolumn{4}{|c}{ Polynomial fit $\left(\rho_{\text {sol }}=\mathrm{a}+\mathrm{b}_{1} \mathrm{x}+\mathrm{b}_{2} \mathrm{x}^{2}+\mathrm{b}_{3} \mathrm{x}^{3}\right)$} \\
& $n_{\text {melt }}$ & $\rho_{\text {melt }} /$ g.cm $^{-3}$ & $\mathrm{a}$ & $\mathrm{b}_{1}$ & $\mathrm{~b}_{2}$ & $\mathrm{~b}_{3}$ \\
\hline \hline Best & 1.5054 & 1.550 & 998.0 & 25.0 & 253.84 & 273.2 \\
\hline Upper & 1.5071 & 1.5565 & 998.0 & 37.88 & 211.13 & 309.49 \\
\hline Lower & 1.5037 & 1.5436 & 998.0 & 12.11 & 296.56 & 236.92 \\
\hline
\end{tabular}

Table S9.2: Tabulated experimental data points shown in Fig S9.1.

\begin{tabular}{ccc|cc}
\hline $\mathrm{a}_{\mathrm{w}}$ & error $\mathrm{a}_{\mathrm{w}}(+\mathrm{ve})$ & error $\mathrm{a}_{\mathrm{w}}(-\mathrm{ve})$ & MFS & error MFS \\
\hline \hline .53894 & 0.0024 & 0.00286 & 0.76226 & 0.00233 \\
0.59688 & 0.01043 & 0.01244 & 0.73375 & 0.00875 \\
0.62961 & 0.00223 & 0.00271 & 0.70793 & $7.62 \mathrm{E}-04$ \\
0.6837 & 0.00876 & 0.01065 & 0.67914 & 0.00409 \\
0.72762 & 0.00123 & 0.00153 & 0.64368 & 0.00135 \\
0.74592 & 0.00403 & 0.00404 & 0.63069 & 0.00342 \\
0.80229 & 0.00504 & 0.0029 & 0.58246 & 0.00401 \\
0.82734 & 0.00237 & 0.00196 & 0.56406 & 0.00314 \\
0.88104 & 0.00107 & 0.0012 & 0.4682 & 0.00149 \\
0.90968 & 0.00331 & 0.00327 & 0.41761 & 0.00738 \\
0.95487 & 0.0028 & 0.00279 & 0.26562 & 0.01165 \\
0.99255 & $6.21 \mathrm{E}-04$ & $6.70 \mathrm{E}-04$ & 0.03973 & 0.00355
\end{tabular}




\section{S10 L-Tartaric Acid Hygroscopicity}

Figure S10.1: Hygroscopicity of Tartaric Acid, (Sigma Aldrich, Purity $\geq 99.5 \%$ ), at 293.15 K. Open squares, these EDB experiments; solid line, UNIFAC model.

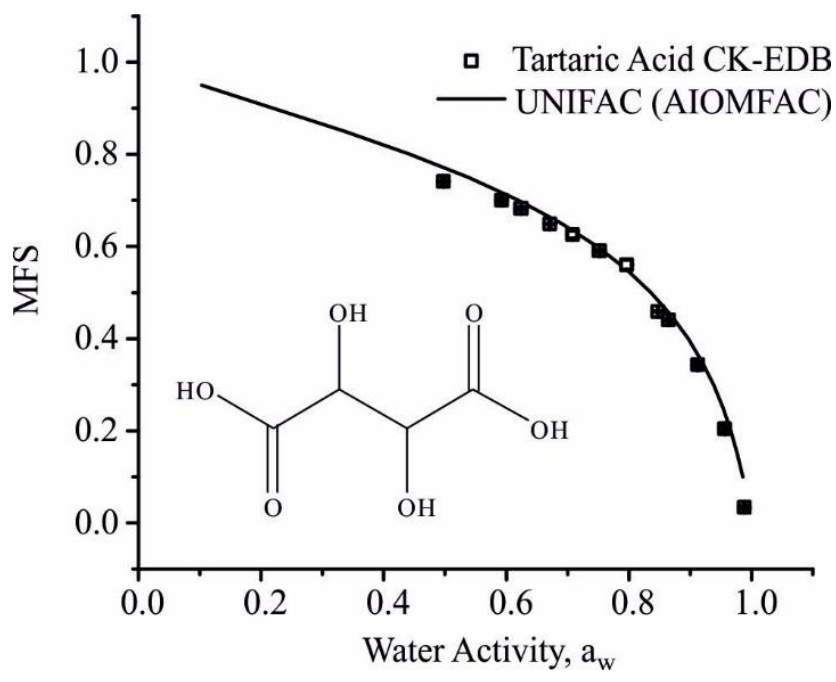

Table S10.1: Pure component refractive index $\left(n_{\text {melt }}\right)$ determined using molar refraction where the melt density $\left(\rho_{\text {melt }}\right)$ is determined using a polynomial fit of density to the square root of MFS $\left(\mathrm{MFS}^{1 / 2}=\mathrm{x}\right)$. Bulk values used are available in Cai et al. (2016). Upper and lower refer to $95 \%$ confidence limits for fits to experimental data.

\begin{tabular}{c|cc|cccc}
\hline & & & \multicolumn{4}{|c}{ Polynomial fit $\left(\rho_{\text {sol }}=\mathrm{a}+\mathrm{b}_{1} \mathrm{x}+\mathrm{b}_{2} \mathrm{x}^{2}+\mathrm{b}_{3} \mathrm{x}^{3}\right)$} \\
& $\mathrm{n}_{\text {melt }}$ & $\rho_{\text {melt }} / \mathrm{g} \cdot \mathrm{cm}^{-3}$ & $\mathrm{a}$ & $\mathrm{b}_{1}$ & $\mathrm{~b}_{2}$ & $\mathrm{~b}_{3}$ \\
\hline \hline Best & 1.4992 & 1.6007 & 999 & 15.08 & 325.84 & 260.78 \\
\hline Upper & 1.4996 & 1.6128 & 999 & 29.23 & 273.11 & 311.49 \\
\hline Lower & 1.4936 & 1.5886 & 999 & 93.2 & 378.58 & 210.06 \\
\hline
\end{tabular}

Table S10.2: Tabulated experimental data points shown in Fig S10.1.

\begin{tabular}{ccc|cc}
\hline $\mathrm{a}_{\mathrm{w}}$ & error $\mathrm{a}_{\mathrm{w}}(+\mathrm{ve})$ & error $\mathrm{a}_{\mathrm{w}}(-\mathrm{ve})$ & MFS & error MFS \\
\hline \hline 0.49764 & 0.00285 & 0.00337 & 0.74075 & 0.00145 \\
0.59229 & 0.00275 & 0.00332 & 0.70005 & 0.00184 \\
0.62457 & 0.00897 & 0.01082 & 0.68273 & 0.00361 \\
0.67107 & 0.00165 & 0.00203 & 0.64893 & $7.95 \mathrm{E}-04$ \\
0.70799 & 0.00711 & 0.00826 & 0.6255 & 0.0076 \\
0.75229 & 0.00853 & 0.01048 & 0.59046 & 0.00337 \\
0.79666 & 0.00778 & 0.00946 & 0.56049 & 0.00992 \\
0.84739 & $9.37 \mathrm{E}-04$ & $5.51 \mathrm{E}-04$ & 0.45906 & 0.00122 \\
0.86463 & 0.00206 & 0.00206 & 0.44068 & 0.00269 \\
0.91248 & 0.00302 & 0.00302 & 0.34362 & 0.00774 \\
0.95599 & 0.00217 & 0.00216 & 0.20415 & 0.00789 \\
0.98847 & 0.00104 & 0.00105 & 0.03363 & 0.00337
\end{tabular}




\section{S11 Oxalic Acid Hygroscopicity}

Fig S11.1: Hygroscopicity of Oxalic Acid, (Sigma Aldrich, Purity $98 \%$ ), at 293.15 K. Open squares, these experiments; solid line, UNIFAC model.
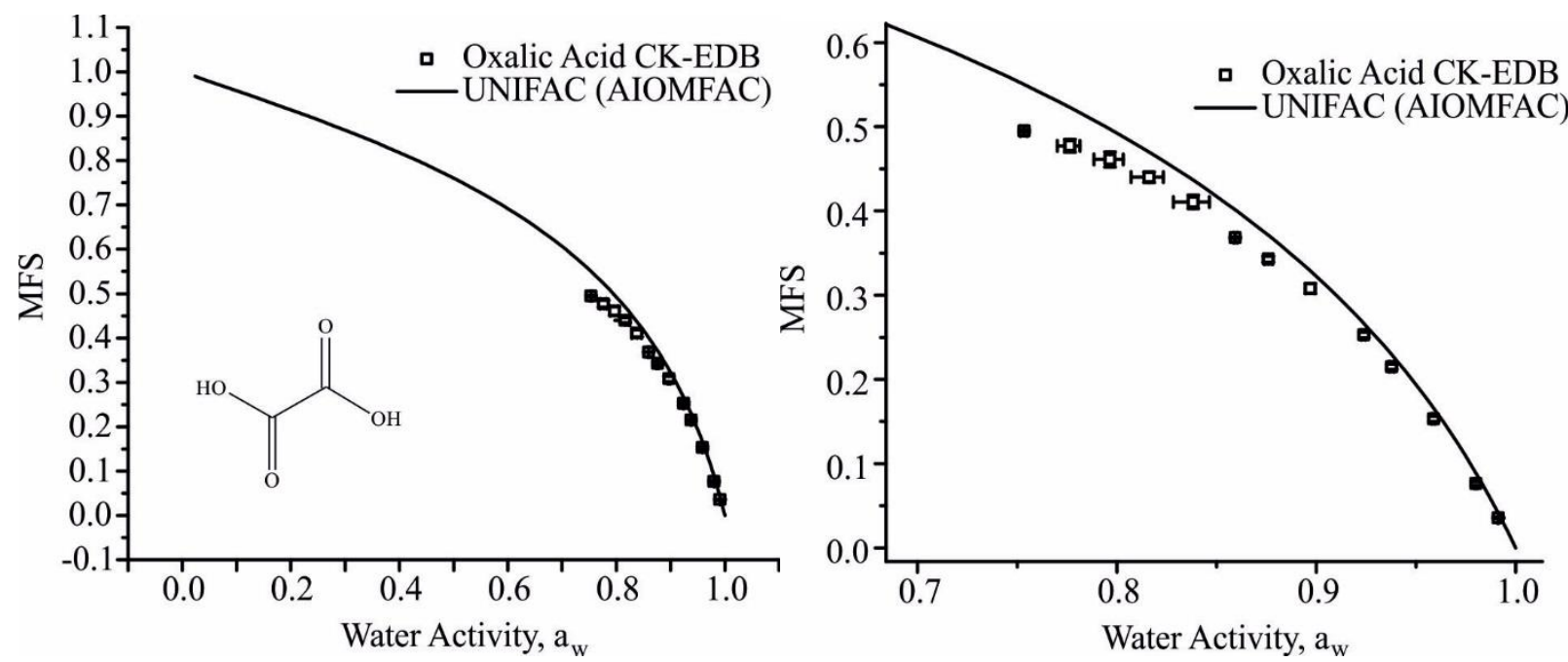

Table S11.1: Pure component refractive index ( $n_{\text {melt }}$ ) is determined using molar refraction, assuming ideal mixing for calculation of the melt density ( $\rho_{\text {melt }}$ ), from bulk data available in Cai et al. (2016). The variation of density as a function of the root of solute mass fraction $\left(\mathrm{MFS}^{1 / 2}=\mathrm{x}\right)$ is represented by polynomial fit parameters. Upper and lower refer to 95 $\%$ confidence limits for fits to experimental data, (Section 2.2 in manuscript).

\begin{tabular}{c|cc|ccccccc}
\hline & & \multicolumn{6}{|c}{ Polynomial fit $\left(\rho_{\text {sol }}=\mathrm{a}+\mathrm{b}_{1} \mathrm{x}+\mathrm{b}_{2} \mathrm{x}^{2}+\mathrm{b}_{3} \mathrm{x}^{3}+\mathrm{b}_{4} \mathrm{x}^{4}+\mathrm{b}_{5} \mathrm{x}^{5}+\mathrm{b}_{6} \mathrm{x}^{6}\right)$} \\
& $n_{\text {melt }}$ & $\begin{array}{c}\rho_{\text {melt }} / \text { g.cm }^{-3} \\
\end{array}$ & $\mathrm{a}$ & $\mathrm{b}_{1}$ & $\mathrm{~b}_{2}$ & $\mathrm{~b}_{3}$ & $\mathrm{~b}_{4}$ & $\mathrm{~b}_{5}$ & $\mathrm{~b}_{6}$ \\
\hline \hline Best & 1.5167 & 1.7237 & 998.4 & -14.98 & 636.47 & -1074.2 & 2603.92 & -2596.5 & 1170.54 \\
\hline Upper & 1.5185 & 1.7403 & 998.4 & -16.27 & 660.48 & -1165.1 & 2811.06 & -2809 & 1260.66 \\
\hline Lower & 1.5149 & 1.7073 & 998.4 & -13.78 & 613.65 & -989.39 & 2409.96 & -2397.5 & 1085.84 \\
\hline
\end{tabular}

Table S11.2: Tabulated experimental data points shown in Fig S11.1.

\begin{tabular}{ccc|cc}
\hline $\mathrm{a}_{\mathrm{w}}$ & error $\mathrm{a}_{\mathrm{w}}(+\mathrm{ve})$ & error $\mathrm{a}_{\mathrm{w}}(-\mathrm{ve})$ & MFS & error MFS \\
\hline \hline 0.75352 & 0.00146 & 0.00183 & 0.49497 & 0.00148 \\
0.77652 & 0.00502 & 0.00629 & 0.47731 & 0.0077 \\
0.79664 & 0.00652 & 0.00817 & 0.46116 & 0.00912 \\
0.81614 & 0.00716 & 0.00896 & 0.44009 & 0.00613 \\
0.83841 & 0.00803 & 0.01005 & 0.41068 & 0.00808 \\
0.85938 & 0.0012 & $7.60 \mathrm{E}-04$ & 0.36829 & 0.00113 \\
0.87602 & 0.00199 & 0.00188 & 0.34275 & 0.00355 \\
0.89702 & 0.00235 & 0.00215 & 0.30804 & 0.00596 \\
0.92388 & $8.50 \mathrm{E}-04$ & 0.00115 & 0.25275 & 0.00331 \\
0.93784 & 0.00106 & 0.00106 & 0.21515 & 0.00299 \\
0.9589 & $8.73 \mathrm{E}-04$ & $8.75 \mathrm{E}-04$ & 0.15314 & 0.00313 \\
0.98012 & $5.72 \mathrm{E}-04$ & $5.68 \mathrm{E}-04$ & 0.07645 & 0.00227 \\
0.99129 & $2.93 \mathrm{E}-04$ & $3.54 \mathrm{E}-04$ & 0.03567 & $8.98 \mathrm{E}-04$ \\
& & & & \\
\hline
\end{tabular}




\section{S12 Malonic Acid Hygroscopicity}

Figure S12.1: Hygroscopicity of Malonic Acid, (Sigma Aldrich, Purity $98 \%$ ), at 293.15 K. Open squares, these experiments; solid line, UNIFAC model.
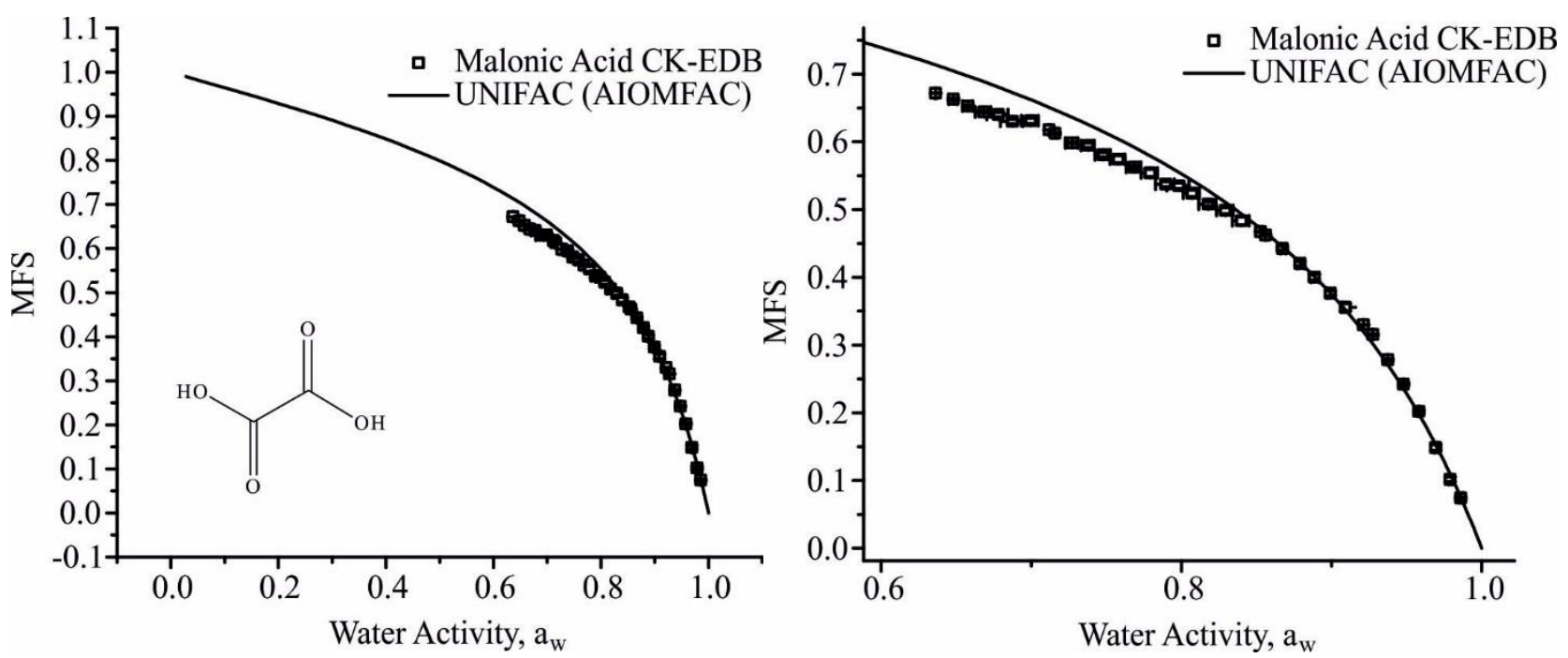

Table S12.1: Pure component refractive index determined using molar refraction where the melt density is determined using a polynomial fit of density to the square root of MFS $\left(\mathrm{MFS}^{1 / 2}=\mathrm{x}\right)$. Bulk values used are available in Cai et al. (2016). Upper and lower refer to $95 \%$ confidence limits for fits to experimental data.

\begin{tabular}{|c|c|c|c|c|c|c|}
\hline & \multirow[b]{2}{*}{$n_{\text {melt }}$} & \multirow[b]{2}{*}{$\rho_{\text {melt }} /$ g. $\mathrm{cm}^{-3}$} & \multicolumn{4}{|c|}{ Polynomial fit $\left(\rho_{\text {sol }}=a+b_{1} x+b_{2} x^{2}+b_{3} x^{3}\right)$} \\
\hline & & & $\mathrm{a}$ & $b_{1}$ & $\mathrm{~b}_{2}$ & $b_{3}$ \\
\hline Best & 1.4611 & 1.4558 & 997.2 & 13.47 & 262.36 & 182.76 \\
\hline Upper & 1.4627 & 1.4612 & 997.2 & 20.7 & 235.91 & 207.37 \\
\hline Lower & 1.4594 & 1.4504 & 997.2 & 6.24 & 288.82 & 158.15 \\
\hline
\end{tabular}

Table S12.2: Tabulated experimental data points shown in Fig S12.1.

\begin{tabular}{ccc|cc}
\hline $\mathrm{a}_{\mathrm{w}}$ & error $\mathrm{a}_{\mathrm{w}}(+\mathrm{ve})$ & error $\mathrm{a}_{\mathrm{w}}(-\mathrm{ve})$ & MFS & error MFS \\
\hline \hline .63613 & $4.14 \mathrm{E}-04$ & $5.04 \mathrm{E}-04$ & 0.6718 & $1.62 \mathrm{E}-04$ \\
0.64803 & 0.00253 & 0.00308 & 0.66275 & 0.00161 \\
0.65776 & 0.00301 & 0.00367 & 0.65273 & 0.00197 \\
0.66822 & 0.00457 & 0.00558 & 0.64421 & 0.00324 \\
0.67795 & 0.00624 & 0.00761 & 0.64043 & 0.00337 \\
0.68747 & 0.00679 & 0.00828 & 0.62992 & 0.00413 \\
0.6994 & 0.0051 & 0.00625 & 0.63085 & $2.85 \mathrm{E}-04$ \\
0.7117 & $4.61 \mathrm{E}-04$ & $5.72 \mathrm{E}-04$ & 0.6176 & 0.00135 \\
0.71572 & 0.00132 & 0.00164 & 0.61275 & 0.00119 \\
0.72728 & 0.00371 & 0.00458 & 0.59849 & 0.00362 \\
0.73786 & 0.00398 & 0.0049 & 0.59485 & 0.00309 \\
0.74792 & 0.00441 & 0.00544 & 0.58075 & 0.00518 \\
0.75777 & 0.00438 & 0.00539 & 0.57442 & 0.00227 \\
0.76852 & 0.00437 & 0.00539 & 0.56217 & 0.00568 \\
0.77901 & 0.00483 & 0.00595 & 0.55399 & 0.0039 \\
0.78948 & 0.00564 & 0.00694 & 0.53743 & 0.00406 \\
0.79831 & 0.00665 & 0.00816 & 0.53501 & 0.00468 \\
0.80703 & 0.00469 & 0.00576 & 0.52388 & 0.00307 \\
0.81779 & 0.00517 & 0.00637 & 0.50809 & 0.00449 \\
0.82931 & 0.00495 & 0.0061 & 0.49855 & 0.0058 \\
0.83997 & 0.00501 & 0.00616 & 0.48341 & $9.32 \mathrm{E}-04$ \\
0.85259 & 0.0013 & $8.20 \mathrm{E}-04$ & 0.46721 & 0.00114 \\
0.85596 & 0.00112 & $6.98 \mathrm{E}-04$ & 0.46233 & 0.00286 \\
0.86726 & 0.00223 & 0.00146 & 0.44257 & 0.0033 \\
0.87898 & 0.00278 & 0.00183 & 0.4203 & 0.0044 \\
0.8885 & 0.00291 & 0.00204 & 0.4002 &
\end{tabular}




\begin{tabular}{ccc|cc}
0.89906 & 0.00294 & 0.00205 & 0.37643 & 0.00371 \\
0.90919 & 0.00337 & 0.00233 & 0.35563 & 0.00559 \\
0.9213 & $1.65 \mathrm{E}-04$ & $2.03 \mathrm{E}-04$ & 0.32987 & $3.33 \mathrm{E}-04$ \\
0.92743 & $2.61 \mathrm{E}-04$ & $2.85 \mathrm{E}-04$ & 0.31545 & 0.00122 \\
0.93737 & $3.90 \mathrm{E}-04$ & $4.01 \mathrm{E}-04$ & 0.27835 & 0.00185 \\
0.94793 & $4.69 \mathrm{E}-04$ & $4.71 \mathrm{E}-04$ & 0.24233 & 0.00205 \\
0.95802 & $4.67 \mathrm{E}-04$ & $4.69 \mathrm{E}-04$ & 0.20227 & 0.00223 \\
0.96932 & 0.0018 & 0.0011 & 0.14857 & 0.00427 \\
0.97897 & 0.00144 & $8.79 \mathrm{E}-04$ & 0.10171 & 0.00358 \\
0.98599 & 0.00158 & $9.62 \mathrm{E}-04$ & 0.07431 & 0.00205 \\
\hline
\end{tabular}

\section{S13 Succinic Acid Hygroscopicity}

Fig S13.1: Hygroscopicity of Succinic Acid, (Sigma Aldrich, Purity 99\%), at 293.15 K. Open squares, these experiments; solid line, UNIFAC model.
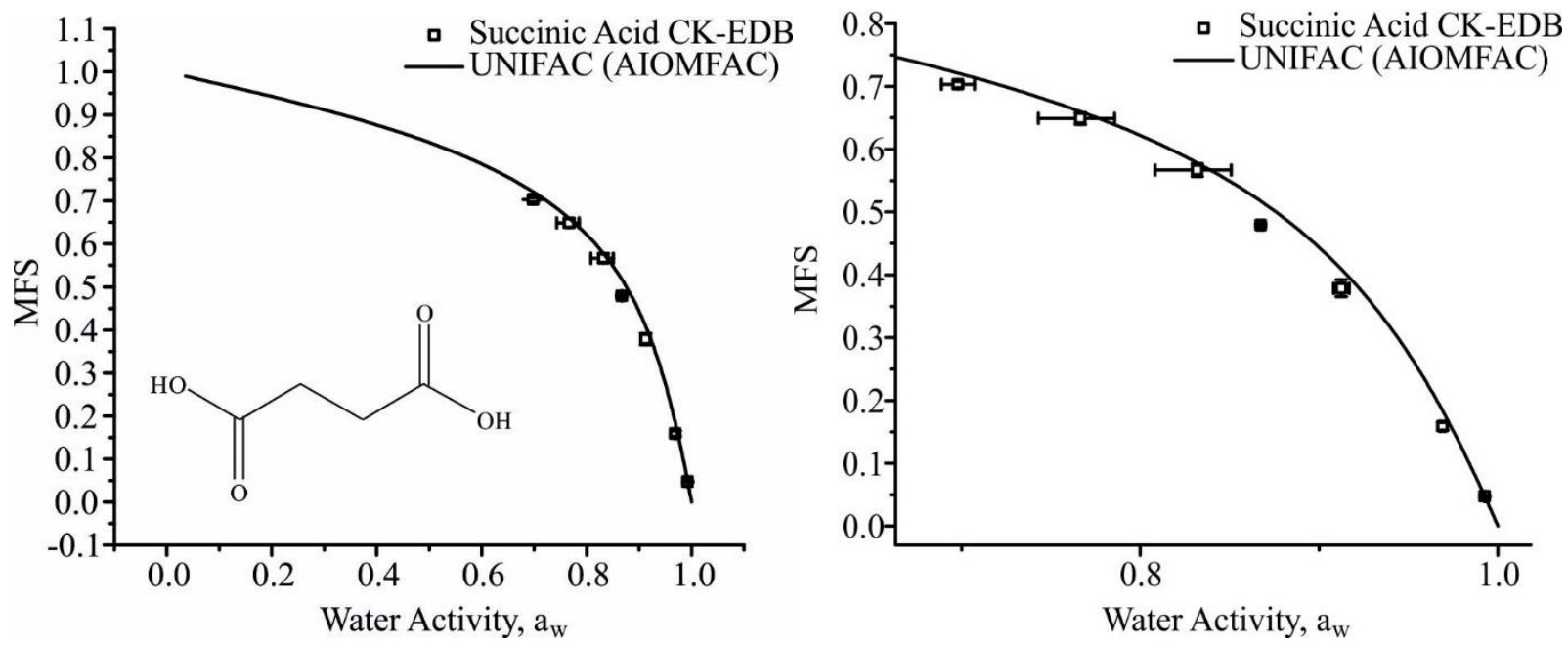

Table S13.1: Pure component refractive index $\left(n_{\text {melt }}\right)$ is determined using molar refraction, assuming ideal mixing for calculation of the melt density $\left(\rho_{\text {melt }}\right)$, from bulk data available in Cai et al. (2016). The variation of density as a function of the root of solute mass fraction $\left(\mathrm{MFS}^{1 / 2}=\mathrm{x}\right.$ ) is represented by polynomial fit parameters. Upper and lower refer to 95 $\%$ confidence limits for fits to experimental data, (Section 2.2 in manuscript).

\begin{tabular}{c|cc|ccccccc}
\hline & & & \multicolumn{5}{|c}{ Polynomial fit $\left(\rho_{\text {sol }}=\mathrm{a}+\mathrm{b}_{1} \mathrm{x}+\mathrm{b}_{2} \mathrm{x}^{2}+\mathrm{b}_{3} \mathrm{x}^{3}+\mathrm{b}_{4} \mathrm{x}^{4}+\mathrm{b}_{5} \mathrm{x}^{5}+\mathrm{b}_{6} \mathrm{x}^{6}\right)$} \\
& $\mathrm{n}_{\text {melt }}$ & $\begin{array}{c}\rho_{\text {melt }} / \\
\mathrm{g.cm}\end{array}$ & $\mathrm{a}$ & $\mathrm{b}_{1}$ & $\mathrm{~b}_{2}$ & $\mathrm{~b}_{3}$ & $\mathrm{~b}_{4}$ & $\mathrm{~b}_{5}$ & $\mathrm{~b}_{6}$ \\
\hline \hline Best & 1.4928 & 1.4185 & 998.2 & -1.96 & 324.69 & -146.48 & 426.3 & -373.62 & 191.37 \\
\hline Upper & 1.4935 & 1.4249 & 998.2 & -2.08 & 329.57 & -155.12 & 447.91 & -395.04 & 201.45 \\
\hline Lower & 1.4920 & 1.4122 & 998.2 & -1.85 & 319.93 & -138.32 & 405.79 & -353.36 & 181.79 \\
\hline
\end{tabular}

Table S13.2: Tabulated experimental data points shown in Fig S13.1.

\begin{tabular}{ccc|cc}
\hline $\mathrm{a}_{\mathrm{w}}$ & error $\mathrm{a}_{\mathrm{w}}(+\mathrm{ve})$ & error $\mathrm{a}_{\mathrm{w}}(-\mathrm{ve})$ & MFS & error MFS \\
\hline \hline 0.69803 & 0.00919 & 0.00918 & 0.70299 & 0.00502 \\
0.76653 & 0.0191 & 0.02355 & 0.64896 & 0.00963 \\
0.83176 & 0.0191 & 0.02355 & 0.56672 & 0.01018 \\
0.86728 & 0.00142 & 0.00142 & 0.47926 & 0.0025 \\
0.91247 & 0.00444 & 0.0044 & 0.37868 & 0.01328 \\
0.96915 & 0.00137 & 0.00136 & 0.15909 & 0.00637 \\
0.99255 & $3.09 \mathrm{E}-04$ & $3.16 \mathrm{E}-04$ & 0.04733 & 0.00178 \\
\end{tabular}




\section{S14 Glutaric Acid Hygroscopicity}

Figure S14.1: Hygroscopicity of Glutaric Acid, (Sigma Aldrich, Purity $99 \%$ ), at 293.15 K. Open squares, these experiments; solid line, UNIFAC model.
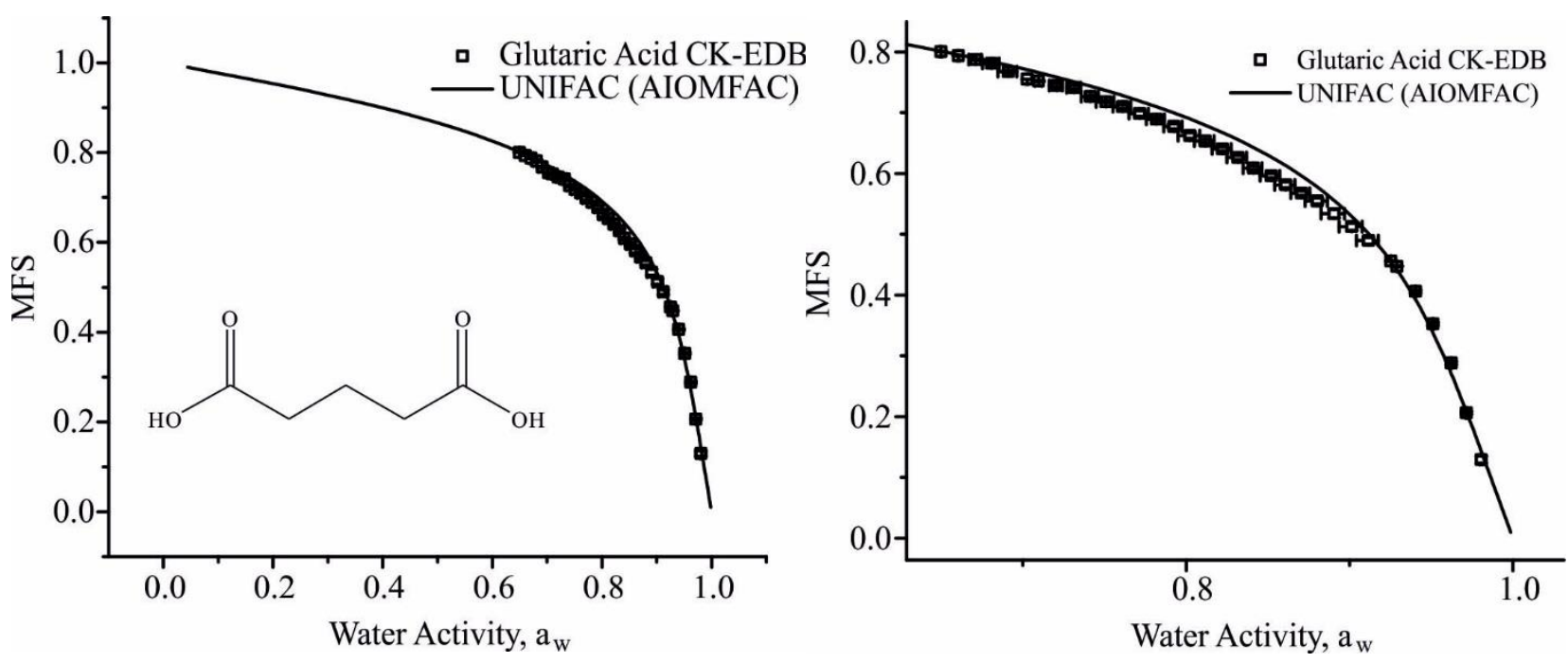

Table S14.1: Pure component refractive index $\left(n_{\text {melt }}\right)$ determined using molar refraction where the melt density $\left(\rho_{\text {melt }}\right)$ is determined using a polynomial fit of density to the square root of MFS $\left(\mathrm{MFS}^{1 / 2}=\mathrm{x}\right)$. Bulk values used are available in Cai et al. (2016). Upper and lower refer to $95 \%$ confidence limits for fits to experimental data.

\begin{tabular}{|c|c|c|c|c|c|c|}
\hline & \multirow[b]{2}{*}{$n_{\text {melt }}$} & \multirow[b]{2}{*}{$\rho_{\text {mell }} /$ g. $\mathrm{cm}^{-3}$} & \multicolumn{4}{|c|}{ Polynomial fit $\left(\rho_{\text {sol }}=a+b_{1} x+b_{2} x^{2}+b_{3} x^{3}\right)$} \\
\hline & & & $\mathrm{a}$ & $b_{1}$ & $\mathrm{~b}_{2}$ & $b_{3}$ \\
\hline Best & 1.4655 & 1.2745 & 9997.5 & -1.56 & 238.79 & 39.75 \\
\hline Upper & 1.4660 & 1.2760 & 997.5 & 0.401 & 231.59 & 46.55 \\
\hline Lower & 1.4649 & 1.2729 & 997.5 & -3.53 & 245.98 & 32.95 \\
\hline
\end{tabular}

Table S14.2: Tabulated experimental data points shown in Fig S14.1.

\begin{tabular}{|c|c|c|c|c|}
\hline $\mathrm{a}_{\mathrm{w}}$ & error $a_{w}(+v e)$ & error $a_{w}(-v e)$ & MFS & error MFS \\
\hline 0.64988 & 4.93E-04 & 6.03E-04 & 0.80052 & $2.01 \mathrm{E}-04$ \\
\hline 0.66053 & $5.88 \mathrm{E}-04$ & $7.21 \mathrm{E}-04$ & 0.79339 & 2.71E-04 \\
\hline 0.67122 & 0.00348 & 0.00426 & 0.78724 & $9.92 \mathrm{E}-04$ \\
\hline 0.68089 & 0.00458 & 0.00561 & 0.78134 & 0.00256 \\
\hline 0.69112 & 0.00522 & 0.00639 & 0.76761 & 0.00226 \\
\hline 0.70268 & $4.02 \mathrm{E}-04$ & 4.98E-04 & 0.75519 & $1.75 \mathrm{E}-04$ \\
\hline 0.70969 & 0.0019 & 0.00235 & 0.75207 & $8.79 \mathrm{E}-04$ \\
\hline 0.72069 & 0.00357 & 0.00441 & 0.74499 & 0.00276 \\
\hline 0.73156 & 0.0038 & 0.00469 & 0.74131 & 0.003 \\
\hline 0.74152 & 0.00467 & 0.00575 & 0.72636 & 0.00217 \\
\hline 0.75089 & 0.00485 & 0.00598 & 0.71793 & 0.00185 \\
\hline 0.76119 & 0.00482 & 0.00594 & 0.70997 & 0.00323 \\
\hline 0.77157 & 0.00535 & 0.00659 & 0.69846 & 0.00478 \\
\hline 0.7818 & 0.00495 & 0.0061 & 0.68945 & 0.00241 \\
\hline 0.79242 & 0.00502 & 0.00618 & 0.6775 & 0.00399 \\
\hline 0.80236 & 5.99E-03 & 7.39E-03 & 0.6624 & 0.00435 \\
\hline 0.81173 & $5.37 \mathrm{E}-03$ & $6.62 \mathrm{E}-03$ & 0.65327 & 0.00271 \\
\hline 0.82228 & 0.00521 & 0.00642 & 0.64016 & 0.00336 \\
\hline 0.83171 & 0.00538 & 0.00662 & 0.62617 & 0.00336 \\
\hline 0.84134 & 0.00521 & 0.00642 & 0.60886 & 0.0028 \\
\hline 0.852 & 0.00538 & 0.00663 & 0.59616 & 0.00364 \\
\hline 0.86106 & 0.00556 & 0.00684 & 0.58136 & 0.00487 \\
\hline 0.87055 & 0.00521 & 0.00642 & 0.56748 & 0.00361 \\
\hline 0.88031 & 0.00617 & 0.00761 & 0.5545 & 0.00465 \\
\hline 0.89058 & 0.00635 & 0.00785 & 0.5337 & 0.00679 \\
\hline 0.90142 & 0.00628 & 0.00777 & 0.51244 & 0.00601 \\
\hline
\end{tabular}




\begin{tabular}{lcc|cc}
0.91173 & 0.00596 & 0.00737 & 0.48949 & 0.00523 \\
0.92543 & $1.50 \mathrm{E}-04$ & $2.02 \mathrm{E}-04$ & 0.45608 & $4.73 \mathrm{E}-04$ \\
0.92905 & $1.56 \mathrm{E}-04$ & $1.58 \mathrm{E}-04$ & 0.44732 & $6.46 \mathrm{E}-04$ \\
0.94053 & $3.86 \mathrm{E}-04$ & $3.86 \mathrm{E}-04$ & 0.40605 & 0.00233 \\
0.95111 & $4.52 \mathrm{E}-04$ & $4.52 \mathrm{E}-04$ & 0.35279 & 0.00256 \\
0.96227 & $3.42 \mathrm{E}-04$ & $3.41 \mathrm{E}-04$ & 0.28818 & 0.00275 \\
0.97153 & $3.20 \mathrm{E}-04$ & $3.20 \mathrm{E}-04$ & 0.20634 & 0.00243 \\
0.98066 & $8.31 \mathrm{E}-04$ & $8.09 \mathrm{E}-04$ & 0.12936 & 0.00558
\end{tabular}

\section{S15 Adipic Acid Hygroscopicity}

Figure S15.1: Hygroscopicity of Adipic Acid, (Sigma Aldrich, Purity $99 \%$ ), at 293.15 K. Open squares, these experiments; solid line, UNIFAC model.
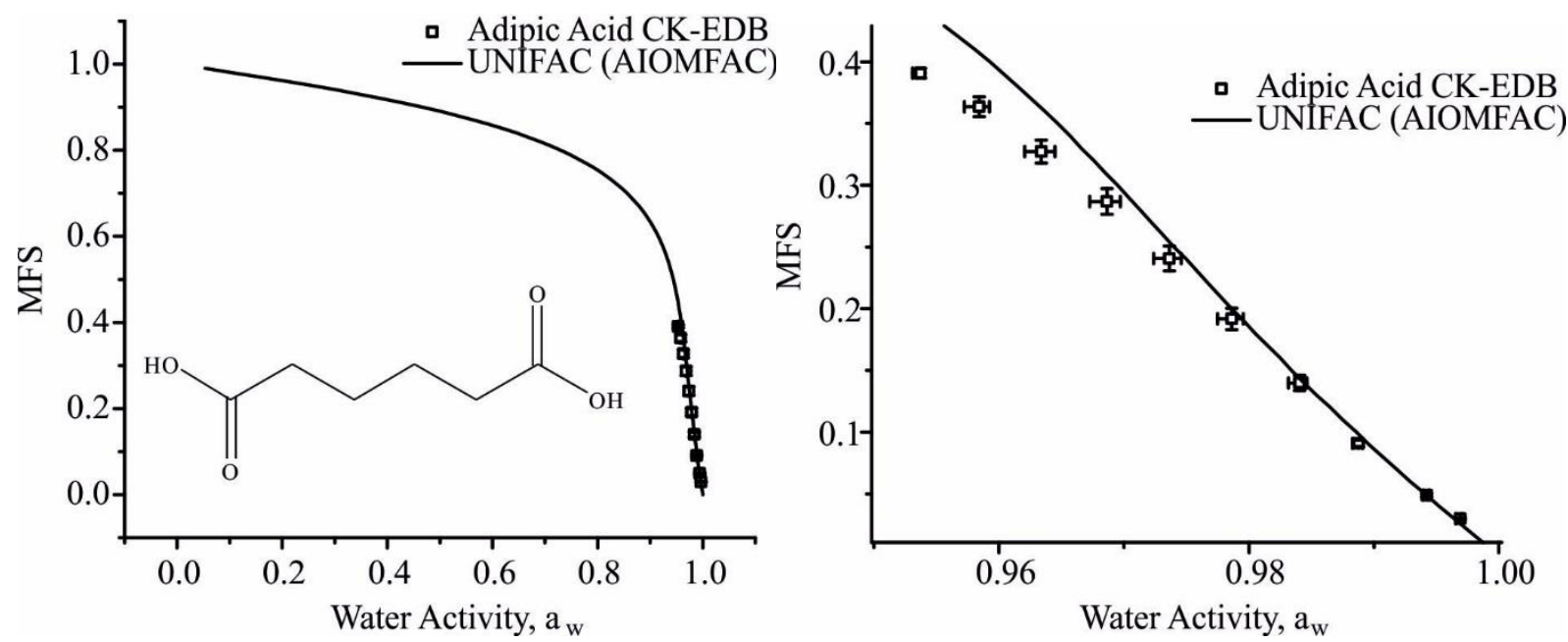

Table S15.1: Pure component refractive index ( $\left.n_{\text {melt }}\right)$ is determined using molar refraction, assuming ideal mixing for calculation of the melt density ( $\rho_{\text {melt }}$ ), from bulk data available in Cai et al. (2016). The variation of density as a function of the root of solute mass fraction $\left(\mathrm{MFS}^{1 / 2}=\mathrm{x}\right)$ is represented by polynomial fit parameters. Upper and lower refer to 95 $\%$ confidence limits for fits to experimental data, (Section 2.2 in manuscript).

\begin{tabular}{c|cc|ccccccc}
\hline & & & \multicolumn{6}{|c}{ Polynomial fit $\left(\rho_{\text {sol }}=\mathrm{a}+\mathrm{b}_{1} \mathrm{x}+\mathrm{b}_{2} \mathrm{x}^{2}+\mathrm{b}_{3} \mathrm{x}^{3}+\mathrm{b}_{4} \mathrm{x}^{4}+\mathrm{b}_{5} \mathrm{x}^{5}+\mathrm{b}_{6} \mathrm{x}^{6}\right)$} \\
& $\mathrm{n}_{\text {melt }}$ & $\begin{array}{c}\rho_{\text {melt }} / \\
\mathrm{g} \mathrm{cm}^{-3}\end{array}$ & $\mathrm{a}$ & $\mathrm{b}_{1}$ & $\mathrm{~b}_{2}$ & $\mathrm{~b}_{3}$ & $\mathrm{~b}_{4}$ & $\mathrm{~b}_{5}$ & $\mathrm{~b}_{6}$ \\
\hline \hline Best & 1.5052 & 1.2897 & 998.2 & -0.483 & 232.81 & -36.78 & 137.06 & -96.59 & 55.48 \\
\hline Upper & 1.5093 & 1.3192 & 998.2 & -0.705 & 253.36 & -53.41 & 183.61 & -139.01 & 77.14 \\
\hline Lower & 1.5012 & 1.2614 & 998.2 & -0.323 & 213.1 & -24.73 & 101.55 & -65.53 & 39.14 \\
\hline
\end{tabular}

Table S15.2: Tabulated experimental data points shown in Fig S15.1.

\begin{tabular}{ccc|cc}
\hline $\mathrm{a}_{\mathrm{w}}$ & error $\mathrm{a}_{\mathrm{w}}(+\mathrm{ve})$ & error $\mathrm{a}_{\mathrm{w}}(-\mathrm{ve})$ & MFS & error MFS \\
\hline \hline 0.95373 & $3.22 \mathrm{E}-04$ & $6.26 \mathrm{E}-04$ & 0.39071 & 0.00391 \\
0.95843 & $8.35 \mathrm{E}-04$ & 0.00118 & 0.36348 & 0.00812 \\
0.9634 & 0.0011 & 0.00133 & 0.3272 & 0.00935 \\
0.96865 & 0.00107 & 0.00138 & 0.28685 & 0.01043 \\
0.97365 & $9.42 \mathrm{E}-04$ & 0.00127 & 0.24062 & 0.01007 \\
0.97863 & $9.10 \mathrm{E}-04$ & 0.00114 & 0.1917 & 0.00876 \\
0.98405 & $5.88 \mathrm{E}-04$ & $8.82 \mathrm{E}-04$ & 0.13977 & 0.00621 \\
0.98877 & $3.13 \mathrm{E}-04$ & $4.91 \mathrm{E}-04$ & 0.09086 & 0.0027 \\
0.99423 & $1.80 \mathrm{E}-04$ & $3.02 \mathrm{E}-04$ & 0.04898 & 0.00153 \\
0.99692 & $1.66 \mathrm{E}-04$ & $3.62 \mathrm{E}-04$ & 0.02978 & $7.74 \mathrm{E}-04$ \\
\hline
\end{tabular}




\section{S16 Pimelic Acid Hygroscopicity}

Fig S16.1: Hygroscopicity of Pimelic Acid, (Sigma Aldrich, Purity 99 \%), at 293.15 K. Open squares, these experiments; solid line, UNIFAC model.
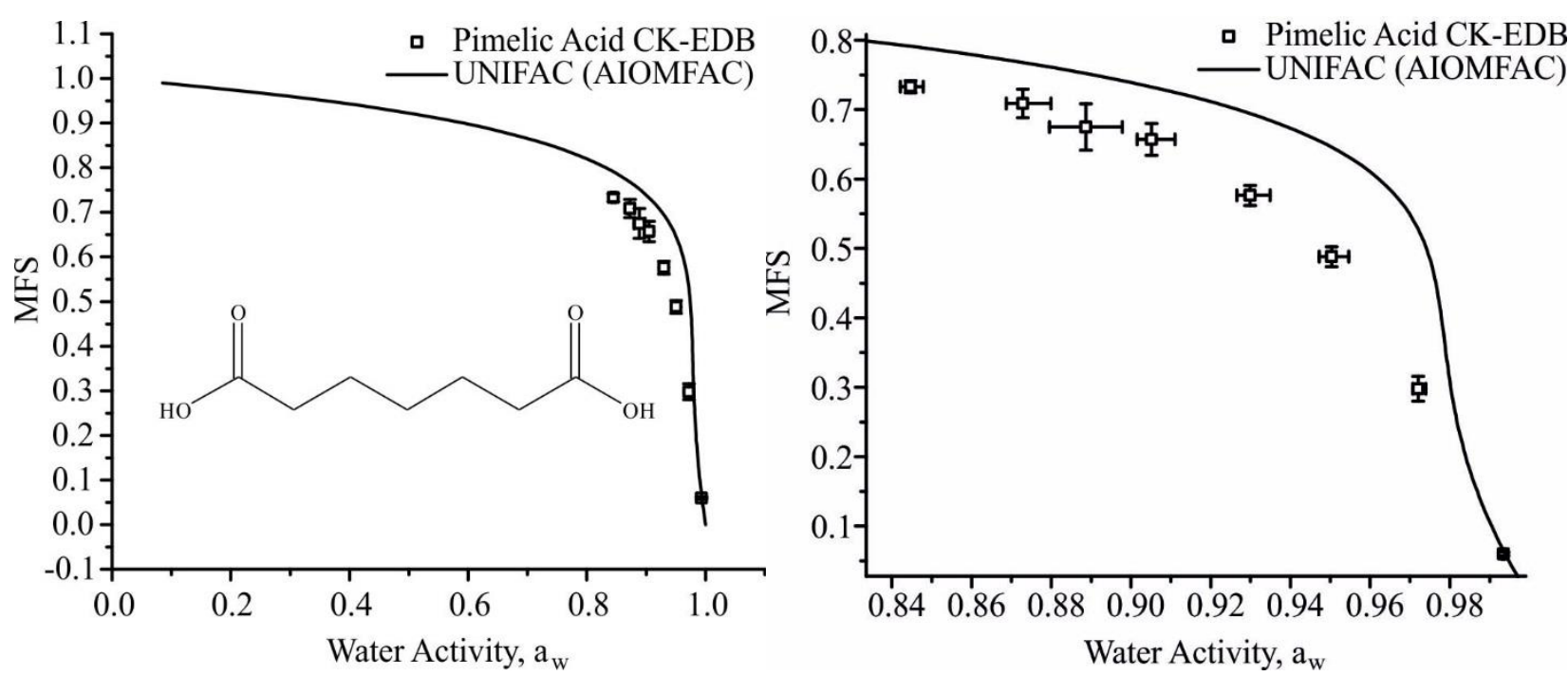

Table S16.1: Pure component refractive index ( $n_{\text {melt }}$ ) is determined using molar refraction, assuming ideal mixing for calculation of the melt density ( $\rho_{\text {melt }}$ ), from bulk data available in Cai et al. (2016). The variation of density as a function of the root of solute mass fraction $\left(\mathrm{MFS}^{1 / 2}=\mathrm{x}\right)$ is represented by polynomial fit parameters. Upper and lower refer to 95 $\%$ confidence limits for fits to experimental data, (Section 2.2 in manuscript).

\begin{tabular}{c|cc|ccccccc}
\hline & & \multicolumn{6}{|c}{ Polynomial fit $\left(\rho_{\text {sol }}=\mathrm{a}+\mathrm{b}_{1} \mathrm{x}+\mathrm{b}_{2} \mathrm{x}^{2}+\mathrm{b}_{3} \mathrm{x}^{3}+\mathrm{b}_{4} \mathrm{x}^{4}+\mathrm{b}_{5} \mathrm{x}^{5}+\mathrm{b}_{6} \mathrm{x}^{6}\right)$} \\
& $\mathrm{n}_{\text {melt }}$ & $\begin{array}{c}\rho_{\text {melt }} / \\
\mathrm{g.cm}\end{array}$ & $\mathrm{a}$ & $\mathrm{b}_{1}$ & $\mathrm{~b}_{2}$ & $\mathrm{~b}_{3}$ & $\mathrm{~b}_{4}$ & $\mathrm{~b}_{5}$ & $\mathrm{~b}_{6}$ \\
\hline \hline Best & 1.4917 & 1.2262 & 998.5 & -0.184 & 188.18 & -14.19 & 67.86 & -37.91 & 23.94 \\
\hline Upper & 1.4940 & 1.2435 & 998.5 & -0.246 & 200.41 & -18.89 & 83.16 & -50.18 & 30.74 \\
\hline Lower & 1.4894 & 1.2095 & 998.5 & -0.136 & 176.23 & -10.52 & 55.25 & -28.29 & 18.47 \\
\hline
\end{tabular}

Table S16.2: Tabulated experimental data points shown in Fig S16.1.

\begin{tabular}{ccc|cc}
\hline $\mathrm{a}_{\mathrm{w}}$ & error $\mathrm{a}_{\mathrm{w}}(+\mathrm{ve})$ & error $\mathrm{a}_{\mathrm{w}}(-\mathrm{ve})$ & MFS & error MFS \\
\hline \hline .84466 & 0.00317 & 0.00251 & 0.73296 & 0.0087 \\
0.87279 & 0.00711 & 0.00413 & 0.70863 & 0.02048 \\
0.88863 & 0.00919 & 0.00916 & 0.67508 & 0.03342 \\
0.90517 & 0.00585 & 0.00361 & 0.65697 & 0.02274 \\
0.92985 & 0.00504 & 0.00334 & 0.57632 & 0.01441 \\
0.9503 & 0.00434 & 0.00304 & 0.48806 & 0.01436 \\
0.97207 & 0.0019 & 0.00139 & 0.29782 & 0.01787 \\
0.99347 & $2.49 \mathrm{E}-04$ & $3.32 \mathrm{E}-04$ & 0.06002 & 0.00268 \\
\end{tabular}




\section{S17 Methyl Malonic Acid Hygroscopicity}

Fig S17.1: Hygroscopicity of methyl malonic acid, (Sigma Aldrich, Purity $99 \%$ ), at 293.15 K. Open squares, these experiments; solid line, UNIFAC model.
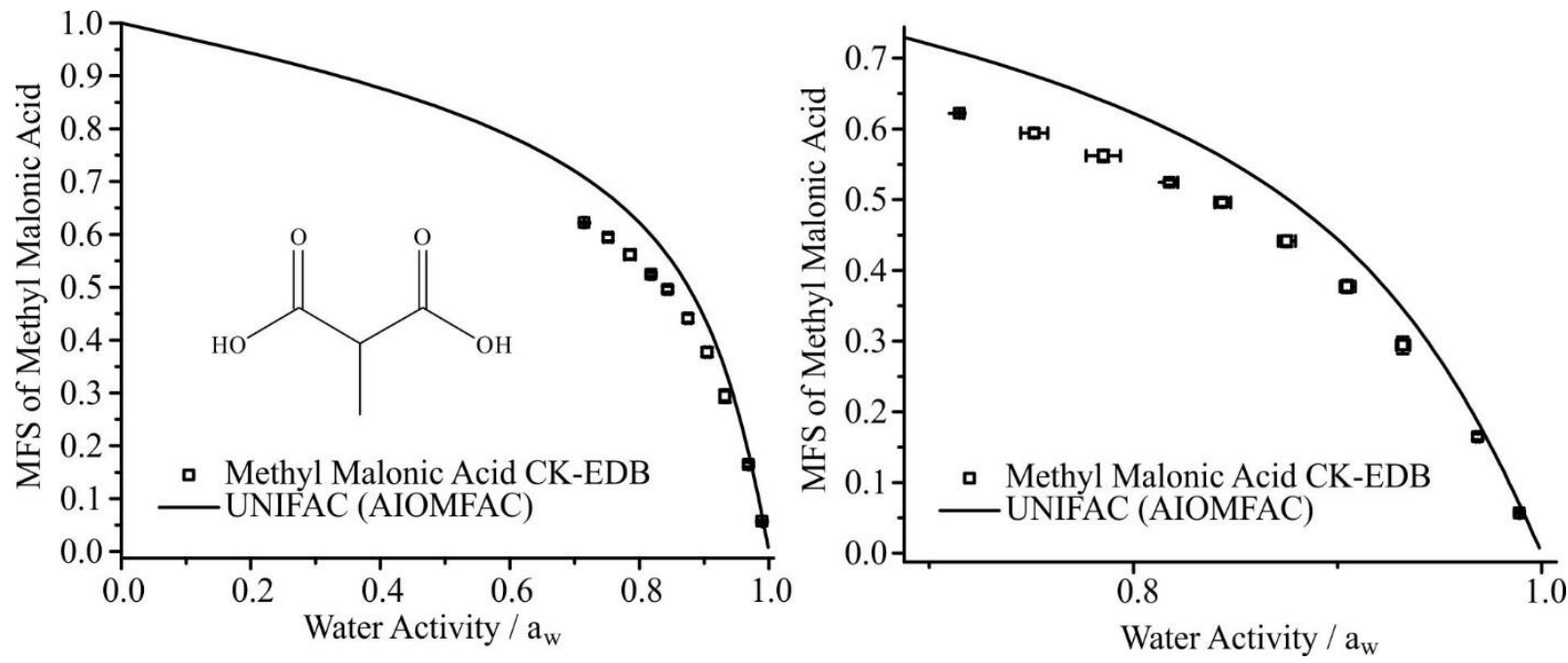

Table S17.1: Pure component refractive index ( $\left.n_{\text {melt }}\right)$ is determined using molar refraction, assuming ideal mixing for calculation of the melt density ( $\rho_{\text {melt }}$ ), from bulk data available in Cai et al. (2016). The variation of density as a function of the root of solute mass fraction $\left(\mathrm{MFS}^{1 / 2}=\mathrm{x}\right)$ is represented by polynomial fit parameters. Upper and lower refer to 95 $\%$ confidence limits for fits to experimental data, (Section 2.2 in manuscript).

\begin{tabular}{|c|c|c|c|c|c|c|c|c|c|}
\hline & \multirow[b]{2}{*}{$n_{\text {melt }}$} & \multirow[b]{2}{*}{$\begin{array}{l}\rho_{\text {mell }}{ }_{\text {g. } \mathrm{cm}^{-3}} \\
\end{array}$} & \multicolumn{7}{|c|}{ Polynomial fit $\left(\rho_{\text {sol }}=a+b_{1} x+b_{2} x^{2}+b_{3} x^{3}+b_{4} x^{4}+b_{5} x^{5}+b_{6} x^{6}\right)$} \\
\hline & & & $\mathrm{a}$ & $b_{1}$ & $\mathrm{~b}_{2}$ & $\mathrm{~b}_{3}$ & $\mathrm{~b}_{4}$ & $b_{5}$ & $\mathrm{~b}_{6}$ \\
\hline Best & 1.4817 & 1.3876 & 998.8 & -1.45 & 301.28 & -108.73 & 330.65 & -279.56 & $1 \overline{146.61}$ \\
\hline Upper & 1.4819 & 1.3902 & 998.8 & -1.49 & 303.18 & -111.53 & 337.82 & -286.56 & 149.98 \\
\hline Lower & 1.4815 & 1.3851 & 998.8 & -1.42 & 299.45 & -106.09 & 323.86 & -272.94 & 143.43 \\
\hline
\end{tabular}

Table S17.2: Tabulated experimental data points shown in Fig S17.1.

\begin{tabular}{ccc|cc}
\hline $\mathrm{a}_{\mathrm{w}}$ & error $\mathrm{a}_{\mathrm{w}}(+\mathrm{ve})$ & error $\mathrm{a}_{\mathrm{w}}(-\mathrm{ve})$ & MFS & error MFS \\
\hline \hline 0.71493 & 0.002 & 0.00248 & 0.62219 & 0.00155 \\
0.75141 & 0.00657 & 0.00657 & 0.59428 & 0.00609 \\
0.78527 & 0.0084 & 0.0084 & 0.562 & 0.00836 \\
0.81777 & 0.004 & 0.00245 & 0.52434 & 0.00364 \\
0.84355 & 0.00409 & 0.00369 & 0.49609 & 0.00573 \\
0.875 & 0.00438 & 0.00401 & 0.44143 & 0.00784 \\
0.90462 & 0.00402 & 0.00333 & 0.3774 & 0.00875 \\
0.93201 & 0.00335 & 0.00317 & 0.29413 & 0.01184 \\
0.96865 & $8.29 \mathrm{E}-04$ & $8.90 \mathrm{E}-04$ & 0.16472 & 0.0041 \\
0.98911 & $4.09 \mathrm{E}-04$ & $4.11 \mathrm{E}-04$ & 0.05691 & 0.00203 \\
& & & & \\
\hline
\end{tabular}




\section{S18 Methyl Succinic Acid Hygroscopicity}

Figure S18.1: Hygroscopicity of methyl succinic acid, (Sigma Aldrich, Purity $99 \%$ ), at 293.15 K. Open squares, these experiments; solid line, UNIFAC model.
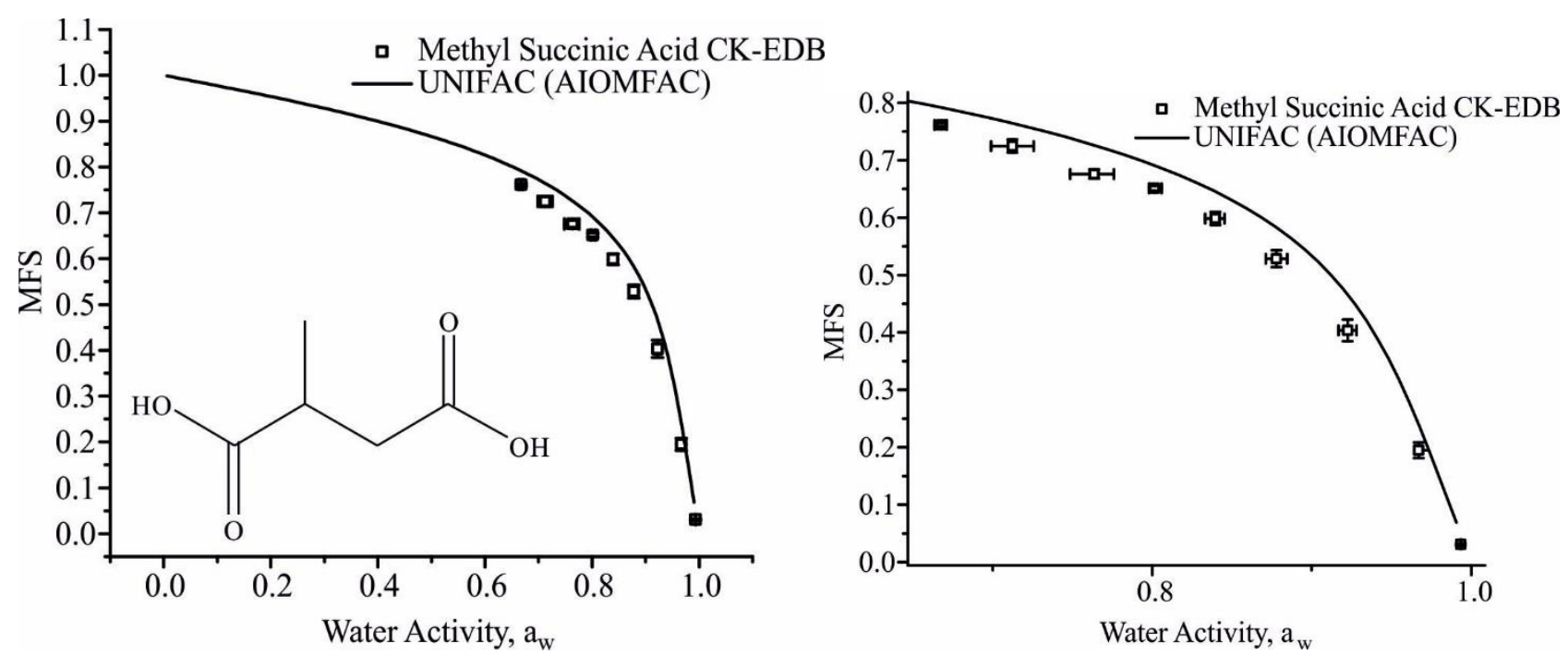

Table S18.1: Pure component refractive index ( $\left.n_{\text {melt }}\right)$ is determined using molar refraction, assuming ideal mixing for calculation of the melt density ( $\rho_{\text {melt }}$ ), from bulk data available in Cai et al. (2016). The variation of density as a function of the root of solute mass fraction $\left(\mathrm{MFS}^{1 / 2}=\mathrm{x}\right.$ ) is represented by polynomial fit parameters. Upper and lower refer to 95 $\%$ confidence limits for fits to experimental data, (Section 2.2 in manuscript).

\begin{tabular}{c|cc|ccccccc}
\hline & & \multicolumn{8}{|c|}{ Polynomial fit $\left(\rho_{\text {sol }}=\mathrm{a}+\mathrm{b}_{1} \mathrm{x}+\mathrm{b}_{2} \mathrm{x}^{2}+\mathrm{b}_{3} \mathrm{x}^{3}+\mathrm{b}_{4} \mathrm{x}^{4}+\mathrm{b}_{5} \mathrm{x}^{5}+\mathrm{b}_{6} \mathrm{x}^{6}\right)$} \\
& $n_{\text {melt }}$ & $\begin{array}{c}\rho_{\text {melt }} \\
\text { g.cm }\end{array}$ & $\mathrm{a}$ & $\mathrm{b}_{1}$ & $\mathrm{~b}_{2}$ & $\mathrm{~b}_{3}$ & $\mathrm{~b}_{4}$ & $\mathrm{~b}_{5}$ & $\mathrm{~b}_{6}$ \\
\hline \hline Best & 1.4779 & 1.3035 & 998.2 & -0.572 & 242.3 & -43.51 & 156.55 & -114.16 & 64.69 \\
\hline Upper & 1.4784 & 1.3090 & 998.2 & -0.614 & 246.13 & -46.62 & 165.26 & -122.12 & 68.76 \\
\hline Lower & 1.4774 & 1.2980 & 998.2 & -0.533 & 238.48 & -40.56 & 148.19 & -106.58 & 60.79 \\
\hline
\end{tabular}

Table S18.2: Tabulated experimental data points shown in Fig S18.1.

\begin{tabular}{ccc|cc}
\hline $\mathrm{a}_{\mathrm{w}}$ & error $\mathrm{a}_{\mathrm{w}}(+\mathrm{ve})$ & error $\mathrm{a}_{\mathrm{w}}(-\mathrm{ve})$ & MFS & error MFS \\
\hline \hline 0.66772 & 0.00345 & 0.00424 & 0.76125 & 0.00296 \\
0.71234 & 0.0134 & 0.0134 & 0.72476 & 0.01132 \\
0.7636 & 0.01237 & 0.01517 & 0.67596 & 0.00785 \\
0.80135 & 0.00451 & 0.00326 & 0.65118 & 0.00447 \\
0.83951 & 0.00575 & 0.00629 & 0.59855 & 0.01151 \\
0.87778 & 0.00688 & 0.00657 & 0.52839 & 0.01469 \\
0.92249 & 0.00567 & 0.00567 & 0.40343 & 0.01891 \\
0.96705 & 0.00282 & 0.00249 & 0.19484 & 0.01368 \\
0.99344 & $3.28 \mathrm{E}-04$ & $3.47 \mathrm{E}-04$ & 0.03075 & 0.00168 \\
& & & & \\
\hline
\end{tabular}




\section{S19 Binary Aqueous Diethylmalonic Acid - Hygroscopicity}

Fig S19.1: Hygroscopicity of diethylmalonic acid, (Sigma Aldrich, Purity $98 \%$ ), at 293.15 K. Open squares, these experiments; solid line, UNIFAC model.
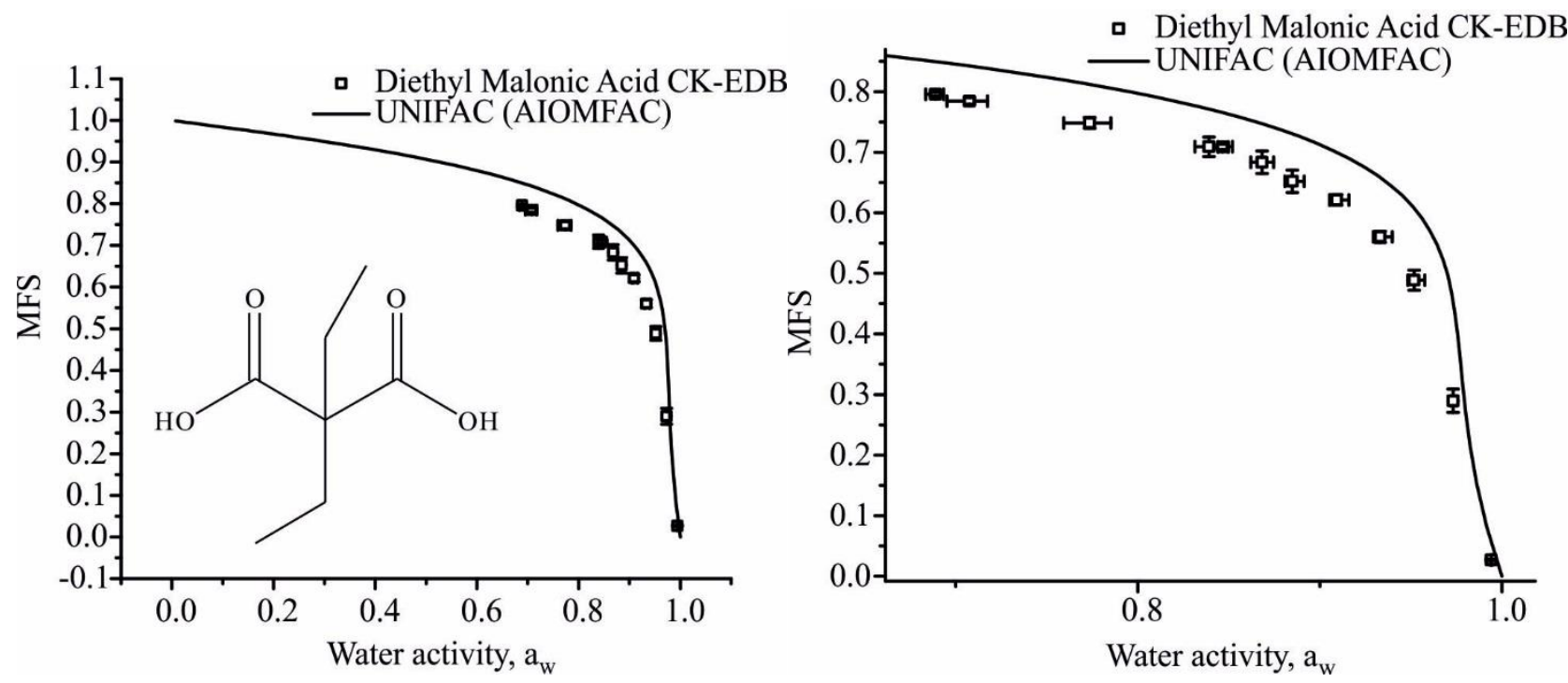

Table S19.1: Pure component refractive index ( $\left.n_{\text {melt }}\right)$ is determined using molar refraction, assuming ideal mixing for calculation of the melt density ( $\rho_{\text {melt }}$ ), from bulk data available in Cai et al. (2016). The variation of density as a function of the root of solute mass fraction $\left(\mathrm{MFS}^{1 / 2}=\mathrm{x}\right.$ ) is represented by polynomial fit parameters. Upper and lower refer to 95 $\%$ confidence limits for fits to experimental data, (Section 2.2 in manuscript).

\begin{tabular}{c|cc|ccccccc}
\hline & & & \multicolumn{5}{|c}{ Polynomial fit $\left(\rho_{\text {sol }}=\mathrm{a}+\mathrm{b}_{1} \mathrm{x}+\mathrm{b}_{2} \mathrm{x}^{2}+\mathrm{b}_{3} \mathrm{x}^{3}+\mathrm{b}_{4} \mathrm{x}^{4}+\mathrm{b}_{5} \mathrm{x}^{5}+\mathrm{b}_{6} \mathrm{x}^{6}\right)$} \\
& $n_{\text {melt }}$ & $\begin{array}{c}\rho_{\text {melt }} \\
\text { g.cm }\end{array}$ & $\mathrm{a}$ & $\mathrm{b}_{1}$ & $\mathrm{~b}_{2}$ & $\mathrm{~b}_{3}$ & $\mathrm{~b}_{4}$ & $\mathrm{~b}_{5}$ & $\mathrm{~b}_{6}$ \\
\hline \hline Best & 1.4854 & 1.2184 & 998.2 & -0.161 & 182.82 & -12.45 & 61.98 & -33.36 & 21.37 \\
\hline Upper & 1.4858 & 1.2219 & 998.2 & -0.172 & 185.32 & -13.25 & 64.69 & -35.44 & 22.55 \\
\hline Lower & 1.4850 & 1.2149 & 998.2 & -0.151 & 180.32 & -11.69 & 59.36 & -31.37 & 20.24 \\
\hline
\end{tabular}

Table S19.2: Tabulated experimental data points shown in Figure S19.1.

\begin{tabular}{ccc|cc}
\hline $\mathrm{a}_{\mathrm{w}}$ & error $\mathrm{a}_{\mathrm{w}}(+\mathrm{ve})$ & error $\mathrm{a}_{\mathrm{w}}(-\mathrm{ve})$ & MFS & error MFS \\
\hline \hline 0.68895 & 0.00441 & 0.00543 & 0.79565 & 0.00315 \\
0.70762 & 0.01 & 0.01233 & 0.78448 & 0.00548 \\
0.7737 & 0.01156 & 0.01425 & 0.7484 & 0.00901 \\
0.83916 & 0.01287 & 0.00773 & 0.70902 & 0.01617 \\
0.84654 & 0.00329 & 0.00246 & 0.70885 & 0.00435 \\
0.86832 & 0.00637 & 0.0062 & 0.68324 & 0.01847 \\
0.88499 & 0.00646 & 0.00418 & 0.65203 & 0.0186 \\
0.90928 & 0.00665 & 0.00391 & 0.62123 & 0.00847 \\
0.93317 & 0.00665 & 0.00374 & 0.56028 & 0.00907 \\
0.95177 & 0.00586 & 0.00329 & 0.48861 & 0.01646 \\
0.97321 & 0.00199 & 0.00152 & 0.28968 & 0.01912 \\
0.99422 & $3.23 \mathrm{E}-04$ & $3.66 \mathrm{E}-04$ & 0.02697 & 0.00157 \\
\hline
\end{tabular}




\section{S20 2,2-Dimethyl Glutaric Acid Hygroscopicity}

Fig S20.1: Hygroscopicity of 2,2-dimethyl glutaric acid, (Sigma Aldrich, Purity > $98 \%$ ), at 293.15 K. Open squares, these experiments; solid line, UNIFAC model.
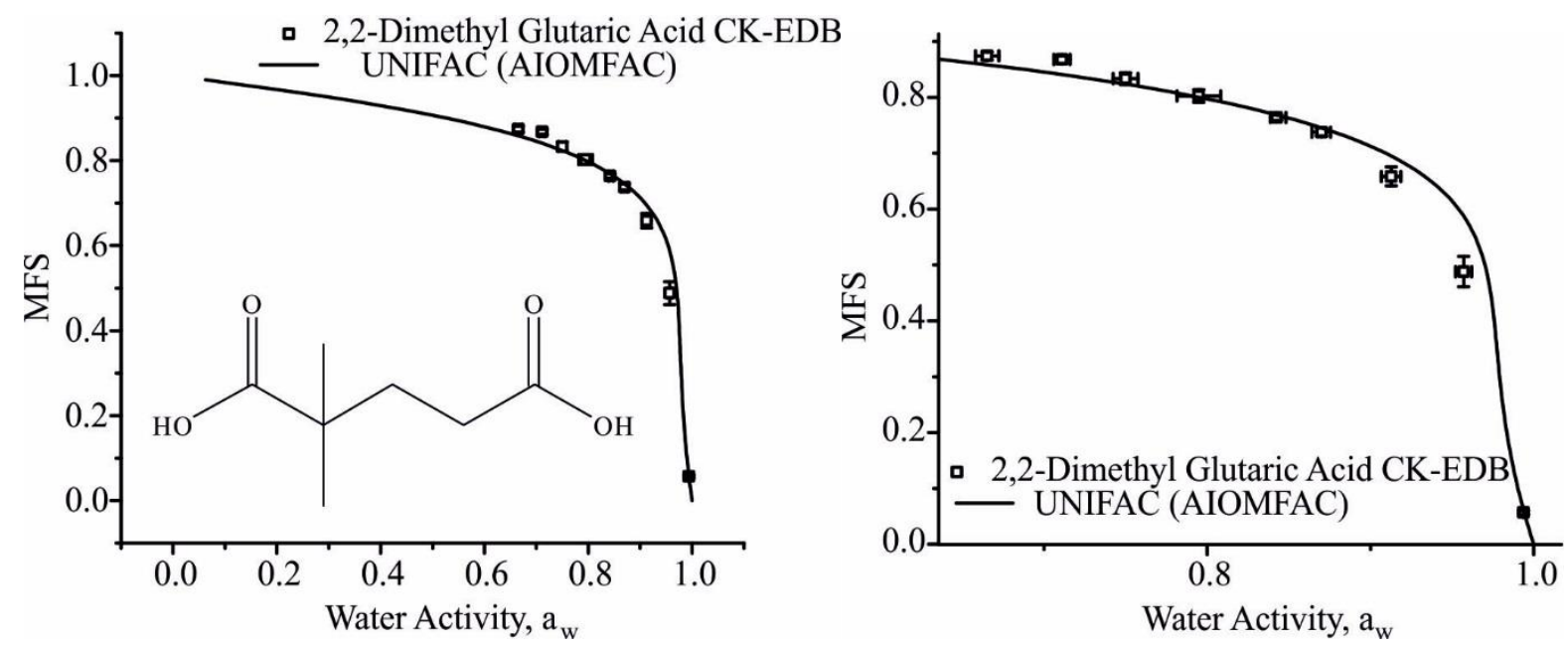

Table S20.1: Pure component refractive index ( $\left.n_{\text {melt }}\right)$ is determined using molar refraction, assuming ideal mixing for calculation of the melt density $\left(\rho_{\text {melt }}\right.$ ), from bulk data available in Cai et al. (2016). The variation of density as a function of the root of solute mass fraction $\left(\mathrm{MFS}^{1 / 2}=\mathrm{x}\right)$ is represented by polynomial fit parameters. Upper and lower refer to 95 $\%$ confidence limits for fits to experimental data, (Section 2.2 in manuscript).

\begin{tabular}{c|cc|ccccccc}
\hline & & \multicolumn{6}{|c}{ Polynomial fit $\left(\rho_{\text {sol }}=\mathrm{a}+\mathrm{b}_{1} \mathrm{x}+\mathrm{b}_{2} \mathrm{x}^{2}+\mathrm{b}_{3} \mathrm{x}^{3}+\mathrm{b}_{4} \mathrm{x}^{4}+\mathrm{b}_{5} \mathrm{x}^{5}+\mathrm{b}_{6} \mathrm{x}^{6}\right)$} \\
& $n_{\text {melt }}$ & $\begin{array}{c}\rho_{\text {mell }} \\
\text { g.cm }\end{array}$ & $\mathrm{a}$ & $\mathrm{b}_{1}$ & $\mathrm{~b}_{2}$ & $\mathrm{~b}_{3}$ & $\mathrm{~b}_{4}$ & $\mathrm{~b}_{5}$ & $\mathrm{~b}_{6}$ \\
\hline \hline Best & 1.4881 & 1.2225 & 998.2 & -0.174 & 185.75 & -13.39 & 65.16 & -35.81 & 22.76 \\
\hline Upper & 1.4884 & 1.2248 & 998.2 & -0.181 & 187.39 & -13.93 & 67 & -37.24 & 23.57 \\
\hline Lower & 1.4878 & 1.2201 & 998.2 & -0.166 & 184.04 & -12.83 & 63.28 & -34.36 & 21.94 \\
\hline
\end{tabular}

Table S19.2: Tabulated experimental data points shown in Fig S20.1.

\begin{tabular}{ccc|cc}
\hline $\mathrm{a}_{\mathrm{w}}$ & error $\mathrm{a}_{\mathrm{w}}(+\mathrm{ve})$ & error $\mathrm{a}_{\mathrm{w}}(-\mathrm{ve})$ & MFS & error MFS \\
\hline \hline 0.66522 & 0.00707 & 0.00713 & 0.87406 & 0.00722 \\
0.71105 & 0.00493 & 0.00494 & 0.8677 & 0.00654 \\
0.74996 & 0.00758 & 0.00758 & 0.83334 & 0.01058 \\
0.79488 & 0.01337 & 0.01338 & 0.80256 & 0.01126 \\
0.84249 & 0.00573 & 0.00389 & 0.76365 & 0.00522 \\
0.86987 & 0.00563 & 0.00574 & 0.73768 & 0.00728 \\
0.91262 & 0.00592 & 0.00605 & 0.65854 & 0.01692 \\
0.95695 & 0.00508 & 0.00491 & 0.48805 & 0.02723 \\
0.99362 & $3.59 \mathrm{E}-04$ & $3.74 \mathrm{E}-04$ & 0.05685 & 0.00348 \\
& & & & \\
\hline
\end{tabular}




\section{S21 2,2-Dimethyl Succinic Acid Hygroscopicity}

Fig S21.1: Hygroscopicity of 2,2-dimethyl succinic acid, (Sigma Aldrich, Purity $99 \%$ ), at 293.15 K. Open squares, these experiments; solid line, UNIFAC model.
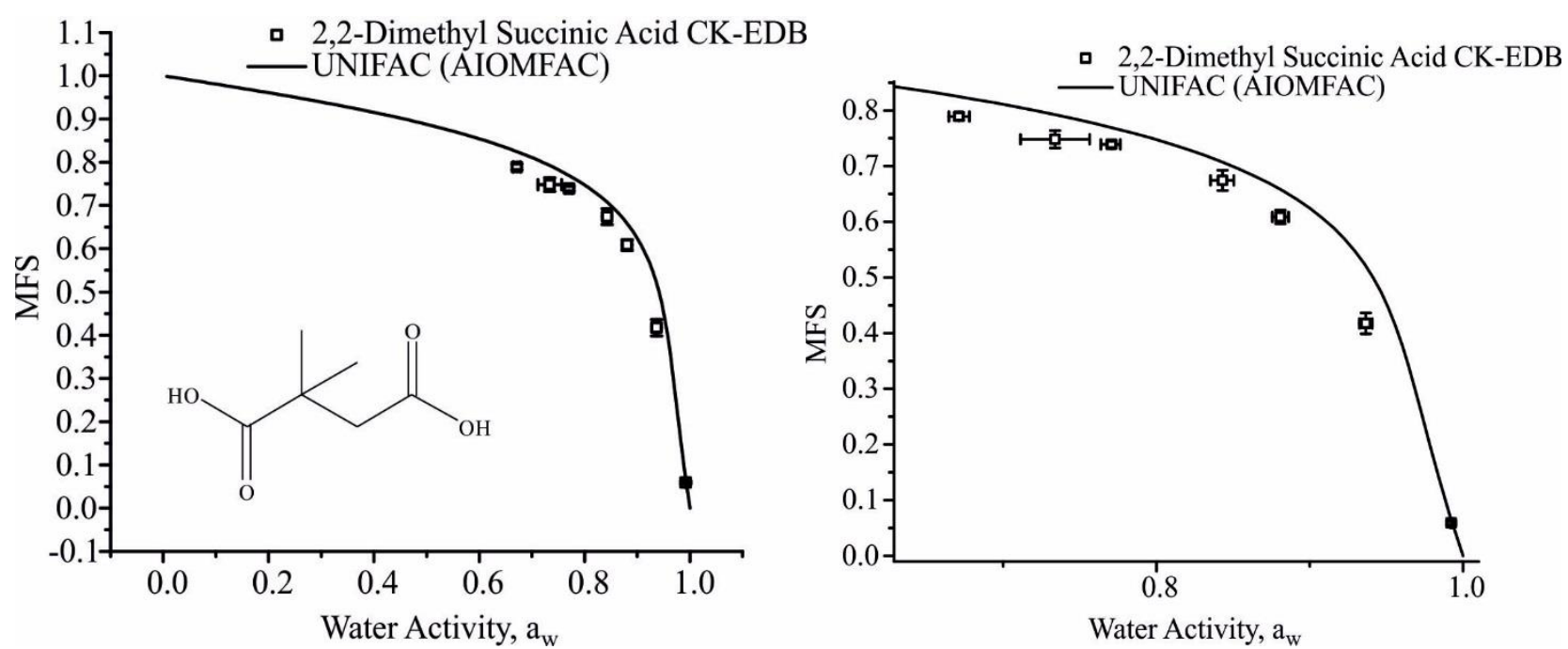

Table S21.1: Pure component refractive index $\left(n_{\text {melt }}\right)$ is determined using molar refraction, assuming ideal mixing for calculation of the melt density $\left(\rho_{\text {melt }}\right.$ ), from bulk data available in Cai et al. (2016). The variation of density as a function of the root of solute mass fraction $\left(\mathrm{MFS}^{1 / 2}=\mathrm{x}\right.$ ) is represented by polynomial fit parameters. Upper and lower refer to 95 $\%$ confidence limits for fits to experimental data, (Section 2.2 in manuscript).

\begin{tabular}{c|cc|ccccccc}
\hline & & \multicolumn{6}{|c}{ Polynomial fit $\left(\rho_{\text {sol }}=\mathrm{a}+\mathrm{b}_{1} \mathrm{x}+\mathrm{b}_{2} \mathrm{x}^{2}+\mathrm{b}_{3} \mathrm{x}^{3}+\mathrm{b}_{4} \mathrm{x}^{4}+\mathrm{b}_{5} \mathrm{x}^{5}+\mathrm{b}_{6} \mathrm{x}^{6}\right)$} \\
& $n_{\text {melt }}$ & $\begin{array}{c}\rho_{\text {melt }}{ }^{-3} \\
\text { g.cm }\end{array}$ & $\mathrm{a}$ & $\mathrm{b}_{1}$ & $\mathrm{~b}_{2}$ & $\mathrm{~b}_{3}$ & $\mathrm{~b}_{4}$ & $\mathrm{~b}_{5}$ & $\mathrm{~b}_{6}$ \\
\hline \hline Best & 1.4889 & 1.2710 & 997.9 & -0.382 & 220.13 & -29.13 & 114.09 & -76.29 & 44.68 \\
\hline Upper & 1.4897 & 1.2776 & 997.9 & -0.419 & 224.73 & -31.96 & 122.4 & -83.53 & 48.48 \\
\hline Lower & 1.4880 & 1.2644 & 997.9 & -0.347 & 215.51 & -26.48 & 106.23 & -69.5 & 41.09 \\
\hline
\end{tabular}

Table S21.2: Tabulated experimental data points shown in Fig S21.1

\begin{tabular}{ccc|cc}
\hline $\mathrm{a}_{\mathrm{w}}$ & error $\mathrm{a}_{\mathrm{w}}(+\mathrm{ve})$ & error $\mathrm{a}_{\mathrm{w}}(-\mathrm{ve})$ & MFS & error MFS \\
\hline \hline 0.6713 & 0.00663 & 0.00663 & 0.78921 & 0.00655 \\
0.73389 & 0.02256 & 0.02256 & 0.74829 & 0.01579 \\
0.77076 & 0.00564 & 0.00705 & 0.73908 & 0.00579 \\
0.84308 & 0.00747 & 0.00776 & 0.67413 & 0.01818 \\
0.88089 & 0.00536 & 0.00529 & 0.60846 & 0.01212 \\
0.9367 & 0.00425 & 0.00424 & 0.41751 & 0.01893 \\
0.99244 & $4.24 \mathrm{E}-04$ & $5.93 \mathrm{E}-04$ & 0.05911 & 0.00313 \\
\end{tabular}




\section{S22 2-Methyl Glutaric Acid Hygroscopicity}

Fig S22.1: Hygroscopicity of 2-methyl glutaric acid, (Sigma Aldrich, Purity $98 \%$ ), at 293.15 K. Open squares, these experiments; solid line, UNIFAC model.
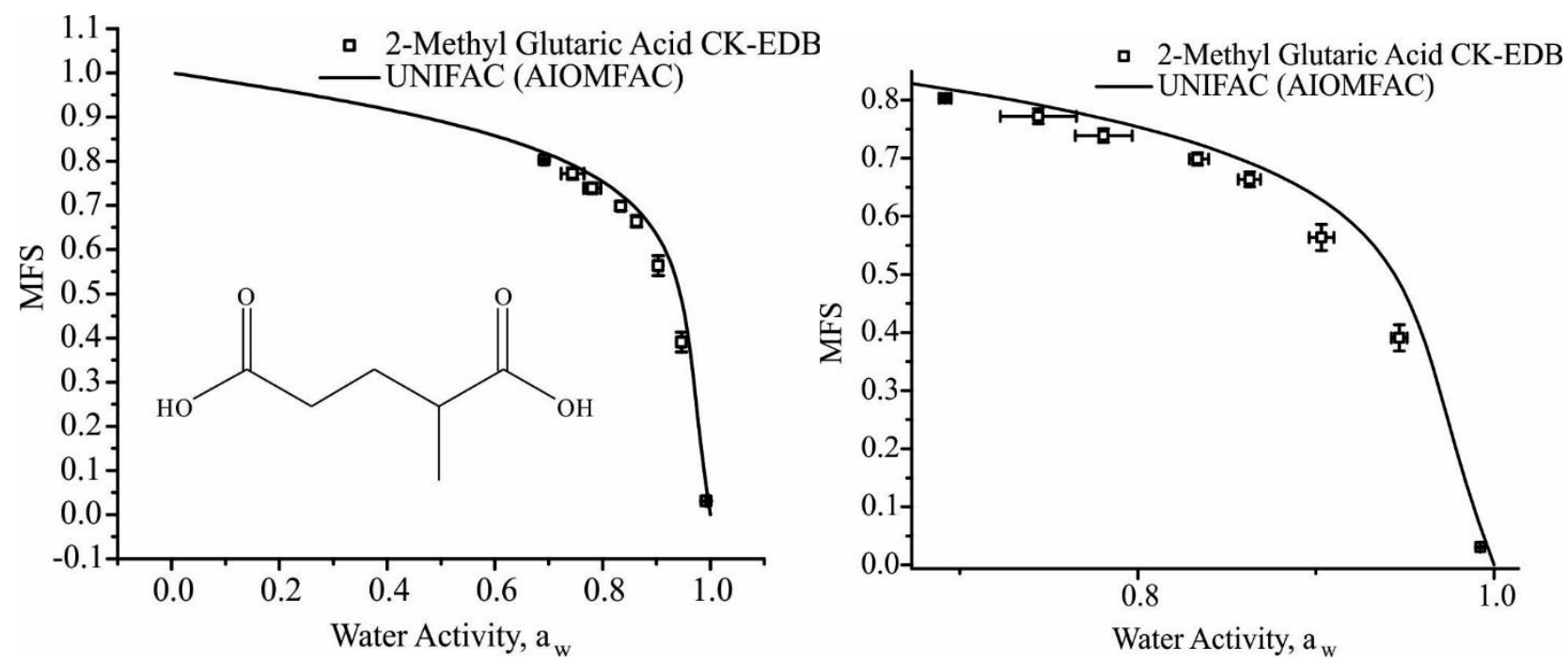

Table S22.1: Pure component refractive index $\left(n_{\text {melt }}\right)$ is determined using molar refraction, assuming ideal mixing for calculation of the melt density $\left(\rho_{\text {melt }}\right)$, from bulk data available in Cai et al. (2016). The variation of density as a function of the root of solute mass fraction $\left(\mathrm{MFS}^{1 / 2}=\mathrm{x}\right)$ is represented by polynomial fit parameters. Upper and lower refer to 95 $\%$ confidence limits for fits to experimental data, (Section 2.2 in manuscript).

\begin{tabular}{c|cc|ccccccc}
\hline & & & \multicolumn{6}{|c}{ Polynomial fit $\left(\rho_{\text {sol }}=\mathrm{a}+\mathrm{b}_{1} \mathrm{x}+\mathrm{b}_{2} \mathrm{x}^{2}+\mathrm{b}_{3} \mathrm{x}^{3}+\mathrm{b}_{4} \mathrm{x}^{4}+\mathrm{b}_{5} \mathrm{x}^{5}+\mathrm{b}_{6} \mathrm{x}^{6}\right)$} \\
& $n_{\text {melt }}$ & $\begin{array}{c}\rho_{\text {melt }} \\
\text { g.cm }\end{array}$ & $\mathrm{a}$ & $\mathrm{b}_{1}$ & $\mathrm{~b}_{2}$ & $\mathrm{~b}_{3}$ & $\mathrm{~b}_{4}$ & $\mathrm{~b}_{5}$ & $\mathrm{~b}_{6}$ \\
\hline \hline Best & 1.4866 & 1.2585 & 997.6 & -0.319 & 211.59 & -24.4 & 99.95 & -64.16 & 38.24 \\
\hline Upper & 1.4873 & 1.2648 & 997.6 & -0.350 & 216 & -26.78 & 107.1 & -70.26 & 41.49 \\
\hline Lower & 1.4858 & 1.2522 & 997.6 & -0.290 & 207.17 & -22.18 & 93.17 & -58.44 & 35.17 \\
\hline
\end{tabular}

Table S22.2: Tabulated experimental data points shown in Fig S22.1

\begin{tabular}{ccc|cc}
\hline $\mathrm{a}_{\mathrm{w}}$ & error $\mathrm{a}_{\mathrm{w}}(+\mathrm{ve})$ & error $\mathrm{a}_{\mathrm{w}}(-\mathrm{ve})$ & MFS & error MFS \\
\hline \hline 0.68925 & 0.00271 & 0.00334 & 0.80479 & 0.00208 \\
0.72204 & 0.01005 & 0.01239 & 0.78383 & 0.00857 \\
0.76123 & 0.01296 & 0.01422 & 0.75567 & 0.01704 \\
0.78959 & 0.02339 & 0.02377 & 0.73478 & 0.02713 \\
0.82836 & 0.01185 & 0.00726 & 0.70077 & 0.02018 \\
0.84699 & 0.00634 & 0.00601 & 0.68658 & 0.01104 \\
0.8785 & 0.00611 & 0.00622 & 0.63205 & 0.01527 \\
0.91076 & 0.00612 & 0.00583 & 0.54437 & 0.02194 \\
0.94004 & 0.00438 & 0.00438 & 0.4312 & 0.02071 \\
0.98128 & $4.79 \mathrm{E}-04$ & 0.0012 & 0.14884 & 0.0113 \\
0.99285 & $2.24 \mathrm{E}-04$ & $2.25 \mathrm{E}-04$ & 0.02928 & 0.00106 \\
\end{tabular}




\section{S23 3-Methyl Adipic Acid Hygroscopicity}

Fig S23.1: Hygroscopicity of 3-methyl adipic acid, (Sigma Aldrich, Purity $99 \%$ ), at 293.15 K. Open squares, these experiments; solid line, UNIFAC model.

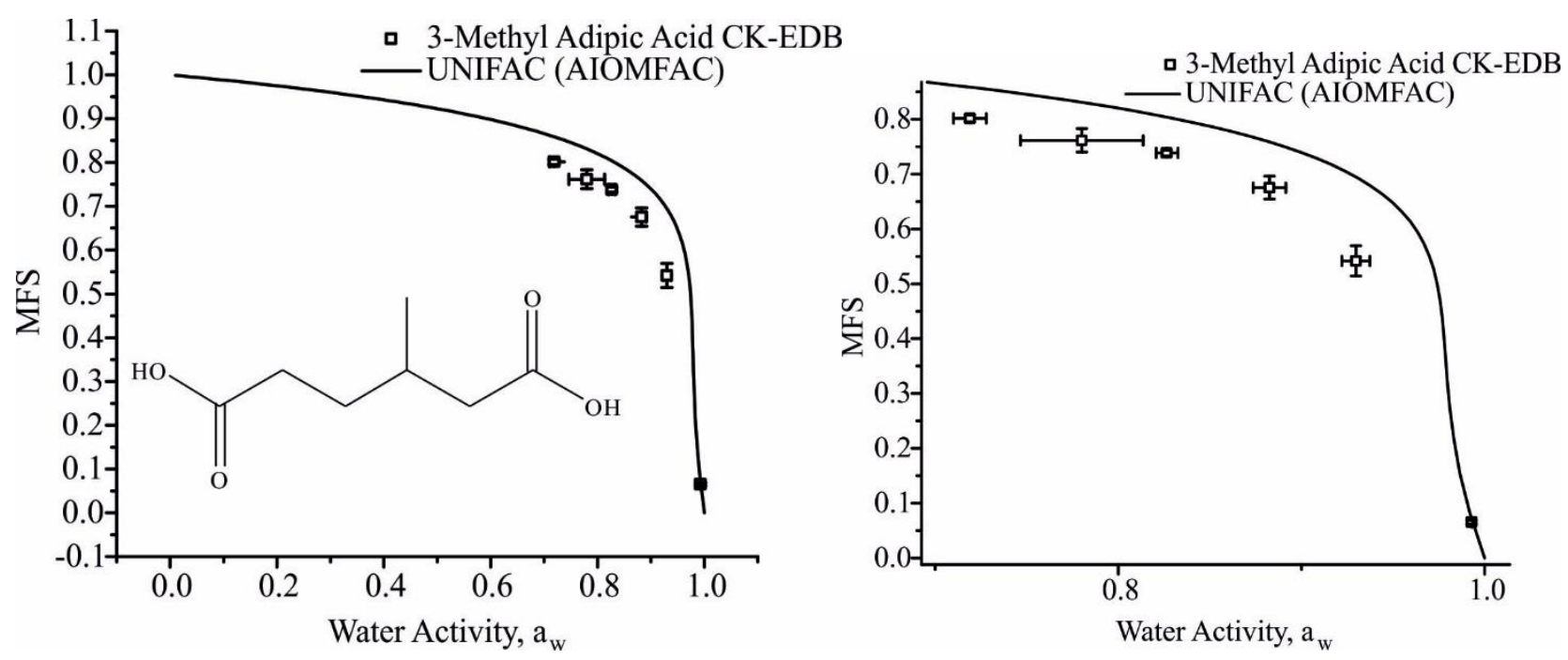

Table S23.1: Pure component refractive index ( $\left.n_{\text {melt }}\right)$ is determined using molar refraction, assuming ideal mixing for calculation of the melt density ( $\rho_{\text {melt }}$ ), from bulk data available in Cai et al. (2016). The variation of density as a function of the root of solute mass fraction $\left(\mathrm{MFS}^{1 / 2}=\mathrm{x}\right)$ is represented by polynomial fit parameters. Upper and lower refer to 95 $\%$ confidence limits for fits to experimental data, (Section 2.2 in manuscript).

\begin{tabular}{c|cc|ccccccc}
\hline & & \multicolumn{6}{|c}{ Polynomial fit $\left(\rho_{\text {sol }}=\mathrm{a}+\mathrm{b}_{1} \mathrm{x}+\mathrm{b}_{2} \mathrm{x}^{2}+\mathrm{b}_{3} \mathrm{x}^{3}+\mathrm{b}_{4} \mathrm{x}^{4}+\mathrm{b}_{5} \mathrm{x}^{5}+\mathrm{b}_{6} \mathrm{x}^{6}\right)$} \\
& $n_{\text {melt }}$ & $\begin{array}{c}\rho_{\text {melt }}{ }^{-3} \\
\text { g.cm }\end{array}$ & $\mathrm{a}$ & $\mathrm{b}_{1}$ & $\mathrm{~b}_{2}$ & $\mathrm{~b}_{3}$ & $\mathrm{~b}_{4}$ & $\mathrm{~b}_{5}$ & $\mathrm{~b}_{6}$ \\
\hline \hline Best & 1.4865 & 1.2141 & 999.0 & -0.147 & 179.19 & -11.33 & 58.11 & -30.42 & 19.69 \\
\hline Upper & 1.4878 & 1.2243 & 999.0 & -0.176 & 186.48 & -13.59 & 65.86 & -36.34 & 23.06 \\
\hline Lower & 1.4852 & 1.2041 & 999.0 & -0.121 & 171.99 & -9.4 & 51.21 & -25.33 & 16.75 \\
\hline
\end{tabular}

Table S23.2: Tabulated experimental data points shown in Fig S23.1.

\begin{tabular}{ccc|cc}
\hline $\mathrm{a}_{\mathrm{w}}$ & error $\mathrm{a}_{\mathrm{w}}(+\mathrm{ve})$ & error $\mathrm{a}_{\mathrm{w}}(-\mathrm{ve})$ & MFS & error MFS \\
\hline \hline 0.71902 & 0.00897 & 0.00897 & 0.80154 & 0.00624 \\
0.78015 & 0.03348 & 0.03347 & 0.7615 & 0.02149 \\
0.82646 & 0.00615 & 0.00574 & 0.73848 & 0.00556 \\
0.88266 & 0.00886 & 0.00907 & 0.67532 & 0.02097 \\
0.92986 & 0.00748 & 0.00771 & 0.54185 & 0.02748 \\
0.993 & $2.61 \mathrm{E}-04$ & $3.72 \mathrm{E}-04$ & 0.06527 & 0.00354 \\
& & & & \\
\hline
\end{tabular}


S24 3-Methyl Glutaric Acid Hygroscopicity

Fig S24.1: Hygroscopicity of 3-methyl glutaric acid, (Sigma Aldrich, Purity $99 \%$ ), at 293.15 K. Open squares, these experiments; solid line, UNIFAC model.
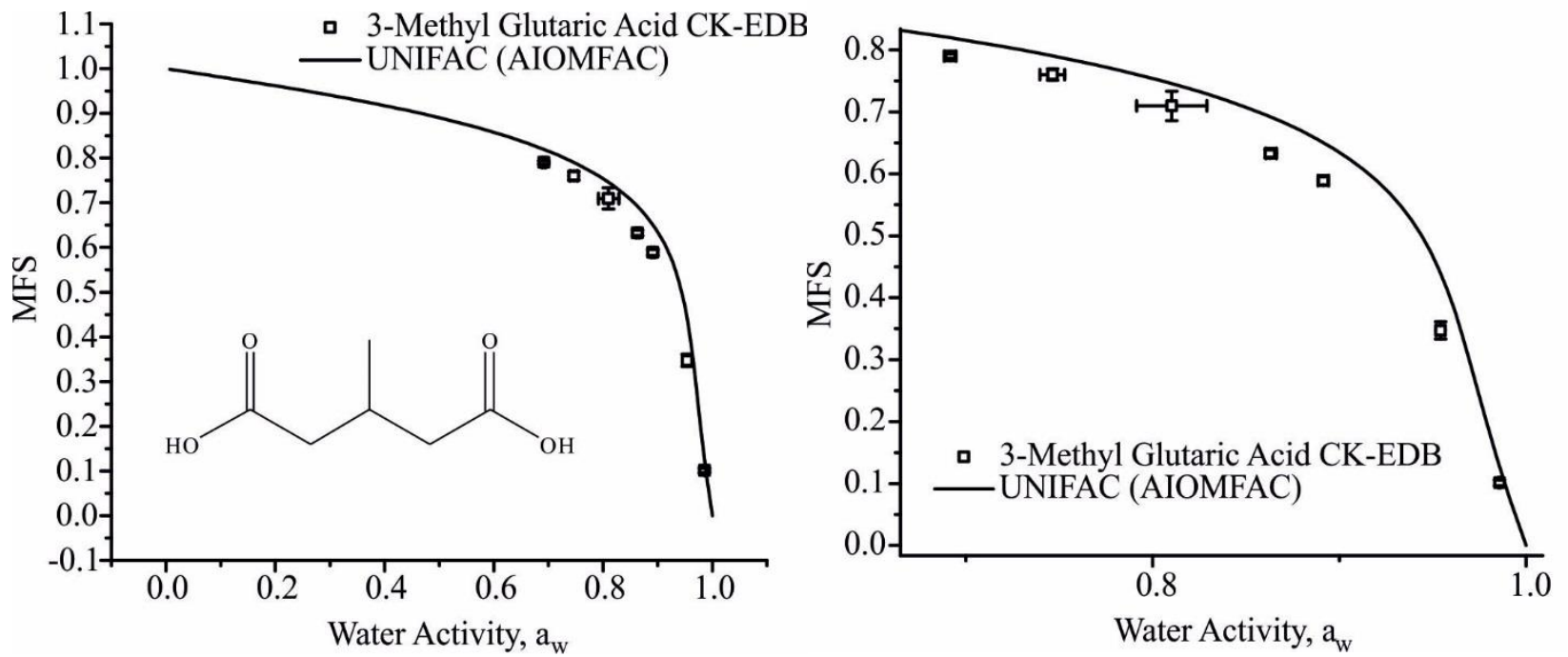

Table SI.24.1: Pure component refractive index $\left(n_{\text {melt }}\right)$ is determined using molar refraction, assuming ideal mixing for calculation of the melt density ( $\rho_{\text {melt }}$ ), from bulk data available in Cai et al. (2016). The variation of density as a function of the root of solute mass fraction $\left(\mathrm{MFS}^{1 / 2}=\mathrm{x}\right)$ is represented by polynomial fit parameters. Upper and lower refer to 95 $\%$ confidence limits for fits to experimental data, (Section 2.2 in manuscript).

\begin{tabular}{c|cc|ccccccc}
\hline & & & \multicolumn{5}{|c}{ Polynomial fit $\left(\rho_{\text {sol }}=\mathrm{a}+\mathrm{b}_{1} \mathrm{x}+\mathrm{b}_{2} \mathrm{x}^{2}+\mathrm{b}_{3} \mathrm{x}^{3}+\mathrm{b}_{4} \mathrm{x}^{4}+\mathrm{b}_{5} \mathrm{x}^{5}+\mathrm{b}_{6} \mathrm{x}^{6}\right)$} \\
& $n_{\text {melt }}$ & $\begin{array}{c}\rho_{\text {melt }} \\
\text { g.cm }\end{array}$ & $\mathrm{a}$ & $\mathrm{b}_{1}$ & $\mathrm{~b}_{2}$ & $\mathrm{~b}_{3}$ & $\mathrm{~b}_{4}$ & $\mathrm{~b}_{5}$ & $\mathrm{~b}_{6}$ \\
\hline \hline Best & 1.4819 & 1.2498 & 997.9 & -0.277 & 205.29 & -21.26 & 90.32 & -56.07 & 33.89 \\
\hline Upper & 1.4822 & 1.2531 & 997.9 & -0.292 & 207.6 & -22.37 & 93.74 & -58.92 & 35.43 \\
\hline Lower & 1.4816 & 1.2466 & 997.9 & -0.264 & 203.04 & -20.22 & 87.1 & -53.39 & 32.44 \\
\hline
\end{tabular}

Table S24.2: Tabulated experimental data points shown in Fig S24.1.

\begin{tabular}{ccc|cc}
\hline $\mathrm{a}_{\mathrm{w}}$ & error $\mathrm{a}_{\mathrm{w}}(+\mathrm{ve})$ & error $\mathrm{a}_{\mathrm{w}}(-\mathrm{ve})$ & MFS & error MFS \\
\hline \hline 0.69173 & 0.00299 & 0.00334 & 0.79013 & 0.0038 \\
0.74649 & 0.00642 & 0.00683 & 0.76025 & 0.00932 \\
0.81013 & 0.01887 & 0.01884 & 0.70959 & 0.02367 \\
0.86283 & 0.00343 & 0.00213 & 0.63276 & 0.00618 \\
0.89131 & 0.00283 & 0.00283 & 0.58884 & 0.00675 \\
0.95411 & 0.00246 & 0.00245 & 0.3472 & 0.01394 \\
0.98567 & $6.06 \mathrm{E}-04$ & $6.09 \mathrm{E}-04$ & 0.10123 & 0.00477 \\
\end{tabular}




\section{S25 3, 3-Dimethyl Glutaric Acid Hygroscopicity}

Fig S25.1: Hygroscopicity of 3, 3-dimethyl glutaric acid, (Sigma Aldrich, Purity 98 \%), at 293.15 K. Open squares, these experiments; solid line, UNIFAC model.

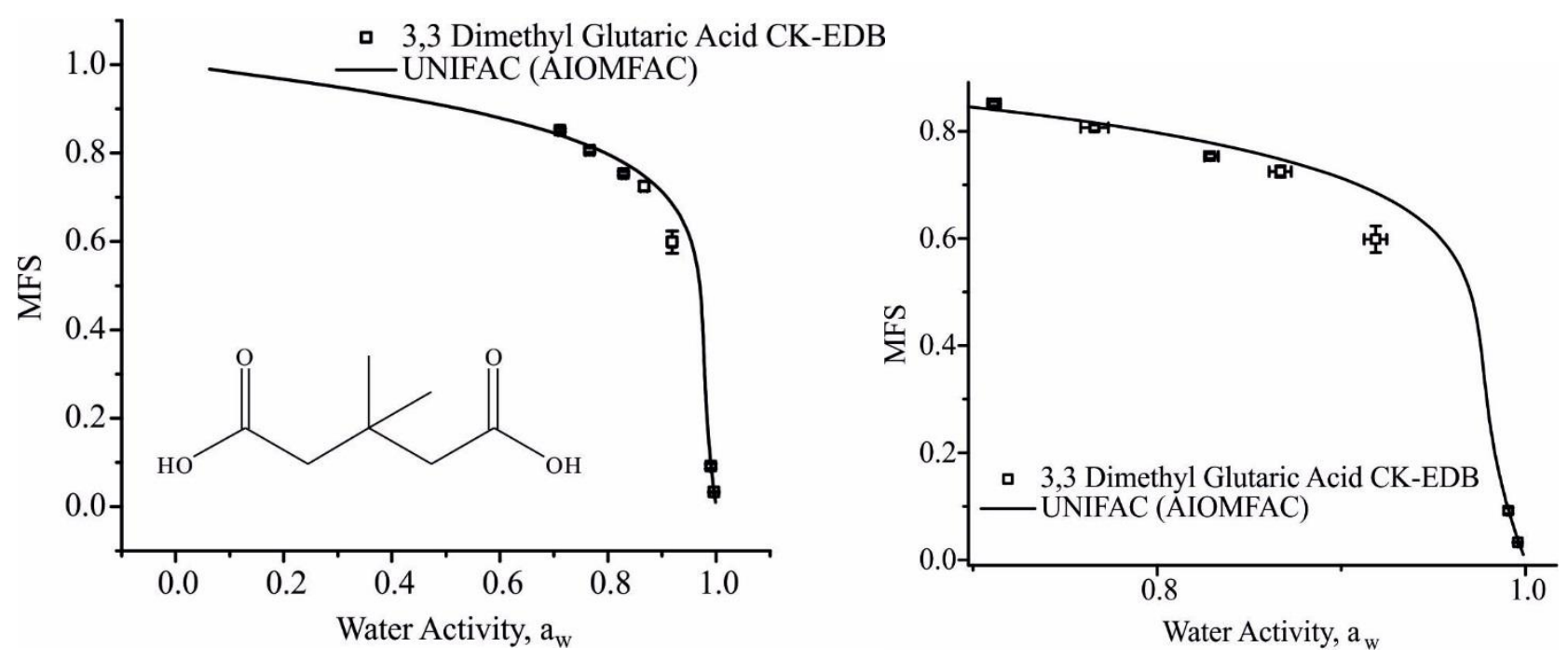

Table S25.1: Pure component refractive index ( $\left.n_{\text {melt }}\right)$ is determined using molar refraction, assuming ideal mixing for calculation of the melt density ( $\rho_{\text {melt }}$ ), from bulk data available in Cai et al. (2016). The variation of density as a function of the root of solute mass fraction $\left(\mathrm{MFS}^{1 / 2}=\mathrm{x}\right)$ is represented by polynomial fit parameters. Upper and lower refer to 95 $\%$ confidence limits for fits to experimental data, (Section 2.2 in manuscript).

\begin{tabular}{c|cc|ccccccc}
\hline & & & \multicolumn{6}{|c}{ Polynomial fit $\left(\rho_{\text {sol }}=\mathrm{a}+\mathrm{b}_{1} \mathrm{x}+\mathrm{b}_{2} \mathrm{x}^{2}+\mathrm{b}_{3} \mathrm{x}^{3}+\mathrm{b}_{4} \mathrm{x}^{4}+\mathrm{b}_{5} \mathrm{x}^{5}+\mathrm{b}_{6} \mathrm{x}^{6}\right)$} \\
& $n_{\text {melt }}$ & $\begin{array}{c}\rho_{\text {melt }} /{ }^{-3} \\
\text { g.cm }\end{array}$ & $\mathrm{a}$ & $\mathrm{b}_{1}$ & $\mathrm{~b}_{2}$ & $\mathrm{~b}_{3}$ & $\mathrm{~b}_{4}$ & $\mathrm{~b}_{5}$ & $\mathrm{~b}_{6}$ \\
\hline \hline Best & 1.4903 & 1.2206 & 998.3 & -0.167 & 184.33 & -12.92 & 63.58 & -34.59 & 22.07 \\
\hline Upper & 1.4906 & 1.2231 & 998.3 & -0.175 & 186.11 & -13.5 & 65.55 & -36.11 & 22.93 \\
\hline Lower & 1.4900 & 1.2182 & 998.3 & -0.160 & 182.61 & -12.38 & 61.74 & -33.18 & 21.27 \\
\hline
\end{tabular}

Table S25.2: Tabulated experimental data points shown in Fig S25.1.

\begin{tabular}{ccc|cc}
\hline $\mathrm{a}_{\mathrm{w}}$ & error $\mathrm{a}_{\mathrm{w}}(+\mathrm{ve})$ & error $\mathrm{a}_{\mathrm{w}}(-\mathrm{ve})$ & MFS & error MFS \\
\hline \hline 0.71132 & 0.00345 & 0.00345 & 0.85176 & 0.00384 \\
0.76078 & 0.006 & 0.00743 & 0.80912 & 0.00721 \\
0.79151 & 0.01941 & 0.01942 & 0.79788 & 0.01562 \\
0.83444 & 0.00416 & 0.00451 & 0.75169 & 0.00421 \\
0.87055 & 0.00543 & 0.00565 & 0.71882 & 0.0105 \\
0.91582 & 0.00545 & 0.00564 & 0.61641 & 0.02163 \\
0.96018 & 0.00389 & 0.00389 & 0.39161 & 0.02576 \\
0.99443 & $2.18 \mathrm{E}-04$ & $2.83 \mathrm{E}-04$ & 0.04485 & 0.00225 \\
& & & & \\
\hline
\end{tabular}


S26. PEG3 Hygroscopicity

Fig S26.1: Hygroscopicity of PEG3, at 293.15 K. Open squares, these experiments; solid line, UNIFAC model.
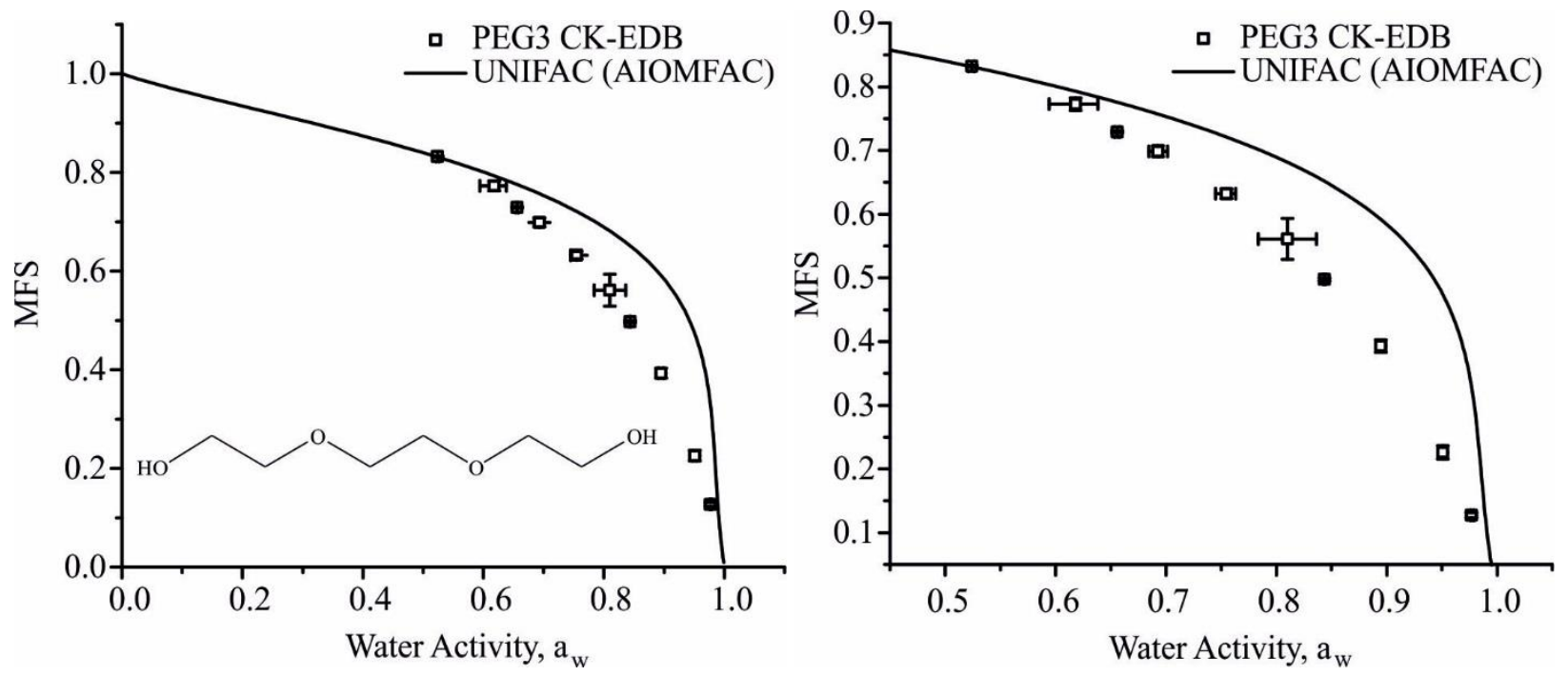

Table S26.1: Measured values of pure component melt density ( $\left.\rho_{\text {melt }}\right)$ and refractive index ( $\left.n_{\text {melt }}\right)$ (PEG3 is liquid), presented with parameterisation for solution measurements of density where $\mathrm{x}$ is the square root of MFS $\left(\mathrm{MFS}^{1 / 2}=\mathrm{x}\right)$. Upper and lower refer to $95 \%$ confidence limits for fits to experimental data. Upper and lower limit on refractive index and density are determined by the error in the refractometer and by the densitometer respectively.

\begin{tabular}{c|cc|cccc}
\hline & & & \multicolumn{4}{|c}{ Polynomial fit $\left(\rho_{\text {sol }}=\mathrm{a}+\mathrm{b}_{1} \mathrm{x}+\mathrm{b}_{2} \mathrm{x}^{2}+\mathrm{b}_{3} \mathrm{x}^{3}\right)$} \\
& $n_{\text {melt }}$ & $\rho_{\text {melt }} / \mathrm{g} . \mathrm{cm}^{-3}$ & $\mathrm{a}$ & $\mathrm{b}_{1}$ & $\mathrm{~b}_{2}$ & $\mathrm{~b}_{3}$ \\
\hline \hline Best & 1.4551 & 1.109 & 999.97 & -75.75 & 431.63 & -246.73 \\
\hline Upper & 1.4552 & 1.122 & 999.97 & -0.198 & 268.11 & -144.15 \\
\hline Lower & 1.4550 & 1.096 & 999.97 & -151.31 & 595.15 & -349.31 \\
\hline
\end{tabular}

Table S26.2: Tabulated experimental data points shown in Fig S26.1.

\begin{tabular}{ccc|cc}
\hline $\mathrm{a}_{\mathrm{w}}$ & error $\mathrm{a}_{\mathrm{w}}(+\mathrm{ve})$ & error $\mathrm{a}_{\mathrm{w}}(-\mathrm{ve})$ & MFS & error MFS \\
\hline \hline 0.524 & 0.0024 & 0.00286 & 0.83232 & 0.00127 \\
0.61806 & 0.02008 & 0.02389 & 0.77269 & 0.0098 \\
0.65597 & 0.00198 & 0.00242 & 0.72923 & 0.00152 \\
0.69291 & 0.00856 & 0.00856 & 0.69867 & 0.0088 \\
0.75489 & $8.16 \mathrm{E}-03$ & 0.01 & 0.63211 & $7.82 \mathrm{E}-03$ \\
0.81001 & 0.0263 & 0.0263 & 0.56113 & 0.03211 \\
0.84347 & 0.00123 & 0.00119 & 0.49753 & 0.00229 \\
0.89472 & 0.00416 & 0.00414 & 0.39303 & 0.01004 \\
0.95087 & $3.07 \mathrm{E}-03$ & $3.07 \mathrm{E}-03$ & 0.22603 & 0.01048 \\
0.97688 & 0.00201 & 0.00112 & 0.12742 & 0.00393 \\
\hline
\end{tabular}




\section{S27. PEG4 Hygroscopicity}

Fig S27.1: Hygroscopicity of PEG4, at 293.15 K. Open squares, these CC-EDB experiments; solid line, UNIFAC model; blue line UManSysProp; red line adsorption isotherm model from Dutcher.

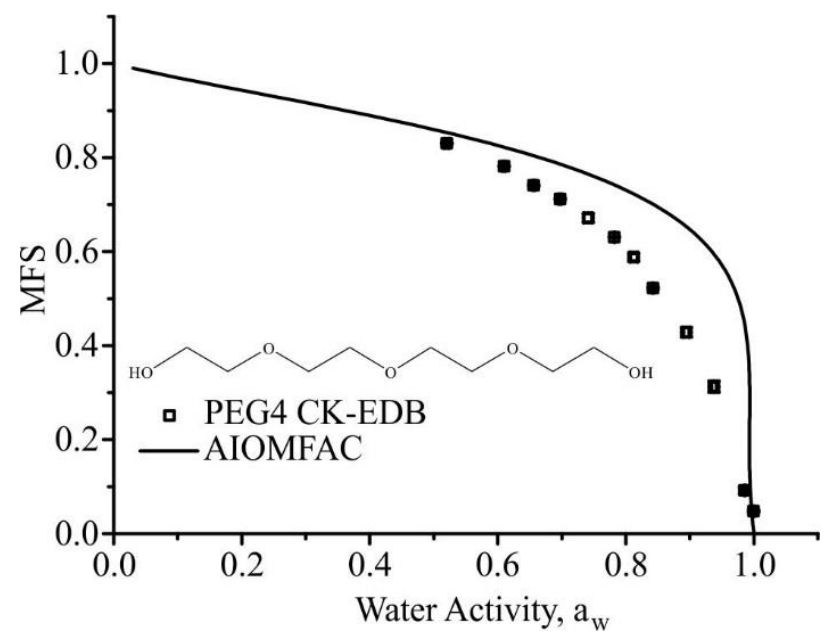

Table S27.1: Measured values of pure component melt density ( $\left.\rho_{\text {melt }}\right)$ and refractive index ( $\left.n_{\text {melt }}\right)$ (PEG4 is liquid), presented with parameterisation for solution measurements of density where $\mathrm{x}$ is the square root of MFS (MFS $\left.{ }^{1 / 2}=\mathrm{x}\right)$. Upper and lower refer to $95 \%$ confidence limits for fits to experimental data. Upper and lower limit on refractive index and density are determined by the error in the refractometer and by the densitometer respectively.

\begin{tabular}{c|cc|cccc}
\hline & & & \multicolumn{4}{|c}{ Polynomial fit $\left(\rho_{\text {sol }}=\mathrm{a}+\mathrm{b}_{1} \mathrm{x}+\mathrm{b}_{2} \mathrm{x}^{2}+\mathrm{b}_{3} \mathrm{x}^{3}\right)$} \\
& $n_{\text {melt }}$ & $\rho_{\text {melt }} /{\mathrm{g} . \mathrm{cm}^{-3}}$ & $\mathrm{a}$ & $\mathrm{b}_{1}$ & $\mathrm{~b}_{2}$ & $\mathrm{~b}_{3}$ \\
\hline \hline Best & 1.4589 & 1.1271 & 999.97 & -37.39 & 296.85 & -130.68 \\
\hline Upper & 1.4590 & 1.13412 & 999.97 & -9.65 & 235.84 & -92.25 \\
\hline Lower & 1.4588 & 1.12338 & 999.97 & -65.13 & 357.86 & -169.11 \\
\hline
\end{tabular}

Table S27.2: Tabulated experimental data points shown in Fig S27.1.

\begin{tabular}{ccc|cc}
\hline $\mathrm{a}_{\mathrm{w}}$ & error $\mathrm{a}_{\mathrm{w}}(+\mathrm{ve})$ & error $\mathrm{a}_{\mathrm{w}}(-\mathrm{ve})$ & MFS & error MFS \\
\hline \hline .52052 & 0.00336 & 0.00399 & 0.83006 & $8.065 \mathrm{E}-4$ \\
0.60966 & 0.00229 & 0.00278 & 0.78149 & $0.220 \mathrm{E}-4$ \\
0.65636 & 0.00166 & 0.00204 & 0.74058 & 0.00157 \\
0.69735 & 0.00172 & 0.00212 & 0.71195 & 0.00929 \\
0.74132 & 0.00556 & 0.00685 & 0.67145 & $8.501 \mathrm{E}-4$ \\
0.78212 & $6.975 \mathrm{E}-4$ & $8.803 \mathrm{E}-4$ & 0.63073 & 0.00759 \\
0.81258 & 0.00536 & 0.00535 & 0.58791 & 0.00213 \\
0.84243 & 0.00132 & 0.00111 & 0.52225 & 0.01048 \\
0.89453 & 0.00427 & 0.00448 & 0.42827 & 0.01217 \\
0.93766 & 0.00385 & 0.00376 & 0.31263 & 0.00662 \\
0.98571 & 0.0013 & 0.00127 & 0.0918 & 0.00252 \\
0.99969 & 0.00143 & 0.00156 & 0.0475 &
\end{tabular}




\section{S28 Erythritol Hygroscopicity}

Fig S28.1: Hygroscopicity of erythritol (Sigma Aldrich 99 \%), at 293.15 K. Open squares, these experiments; solid line, UNIFAC model.

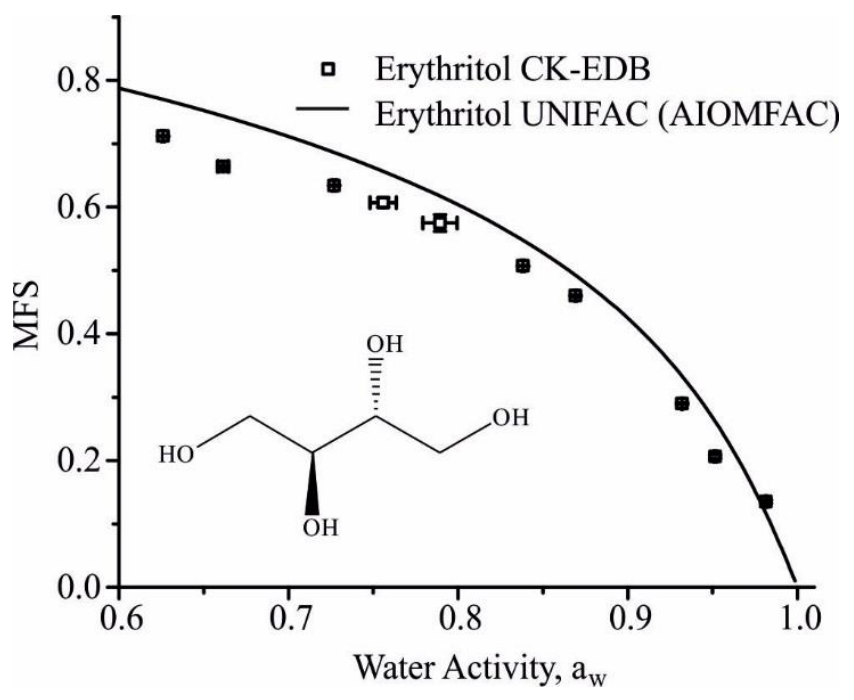

Table S28.1: Pure component refractive index $\left(n_{\text {melt }}\right)$ is determined using molar refraction, assuming ideal mixing for calculation of the melt density $\left(\rho_{\text {melt }}\right)$, from bulk data available in Cai et al. (2016). The variation of density as a function of the root of solute mass fraction $\left(\mathrm{MFS}^{1 / 2}=\mathrm{x}\right)$ is represented by polynomial fit parameters. Upper and lower refer to 95 $\%$ confidence limits for fits to experimental data, (Section 2.2 in manuscript).

\begin{tabular}{c|cc|cccc}
\hline & & & \multicolumn{4}{|c}{ Polynomial fit $\left(\rho_{\text {sol }}=\mathrm{a}+\mathrm{b}_{1} \mathrm{x}+\mathrm{b}_{2} \mathrm{x}^{2}+\mathrm{b}_{3} \mathrm{x}^{3}\right)$} \\
& $n_{\text {melt }}$ & $\rho_{\text {melt }} /{\mathrm{g} . \mathrm{cm}^{-3}}$ & $\mathrm{a}$ & $\mathrm{b}_{1}$ & $\mathrm{~b}_{2}$ & $\mathrm{~b}_{3}$ \\
\hline \hline Best & 1.5211 & 1.3754 & 998.6 & 58.46 & 37.98 & 278.66 \\
\hline Upper & 1.5388 & 1.3813 & 998.6 & 60.21 & 33.79 & 286.94 \\
\hline Lower & 1.5204 & 1.3695 & 998.6 & 56.75 & 42.03 & 27.049 \\
\hline
\end{tabular}

Table S28.2: Tabulated experimental data points shown in Fig S28.1.

\begin{tabular}{ccc|cc}
\hline $\mathrm{a}_{\mathrm{w}}$ & error $\mathrm{a}_{\mathrm{w}}(+\mathrm{ve})$ & error $\mathrm{a}_{\mathrm{w}}(-\mathrm{ve})$ & MFS & error MFS \\
\hline \hline 0.62602 & $8.77112 \mathrm{E}-4$ & 0.00107 & 0.71188 & $6.08334 \mathrm{E}-4$ \\
0.66147 & 0.0027 & 0.0033 & 0.66395 & 0.00226 \\
0.72692 & 0.00104 & 0.00129 & 0.6342 & $6.57702 \mathrm{E}-4$ \\
0.75582 & 0.00775 & 0.00777 & 0.60723 & 0.00739 \\
0.78929 & 0.01009 & 0.0101 & 0.57499 & 0.01315 \\
0.83827 & $7.77253 \mathrm{E}-4$ & 0.001 & 0.50705 & $9.72437 \mathrm{E}-4$ \\
0.86916 & $7.13427 \mathrm{E}-4$ & $6.96511 \mathrm{E}-4$ & 0.46004 & 0.00138 \\
0.93195 & $2.64028 \mathrm{E}-4$ & $3.52642 \mathrm{E}-4$ & 0.28987 & 0.00175 \\
0.95145 & $7.53773 \mathrm{E}-4$ & $7.52526 \mathrm{E}-4$ & 0.20621 & 0.00312 \\
0.9815 & $5.76107 \mathrm{E}-4$ & $5.56581 \mathrm{E}-4$ & 0.13503 & 0.00279 \\
\end{tabular}




\section{S29 Sorbitol Hygroscopicity}

Fig S29.1: Hygroscopicity of sorbitol (Sigma Aldrich $\geq 98 \%$ ), at 293.15 K. Open squares, these experiments; solid line, UNIFAC model. Data taken at RHs lower than indicated by the dashed black line show increased error in hygroscopicity retrieval due to the imposition of a kinetic limitation on water transport.

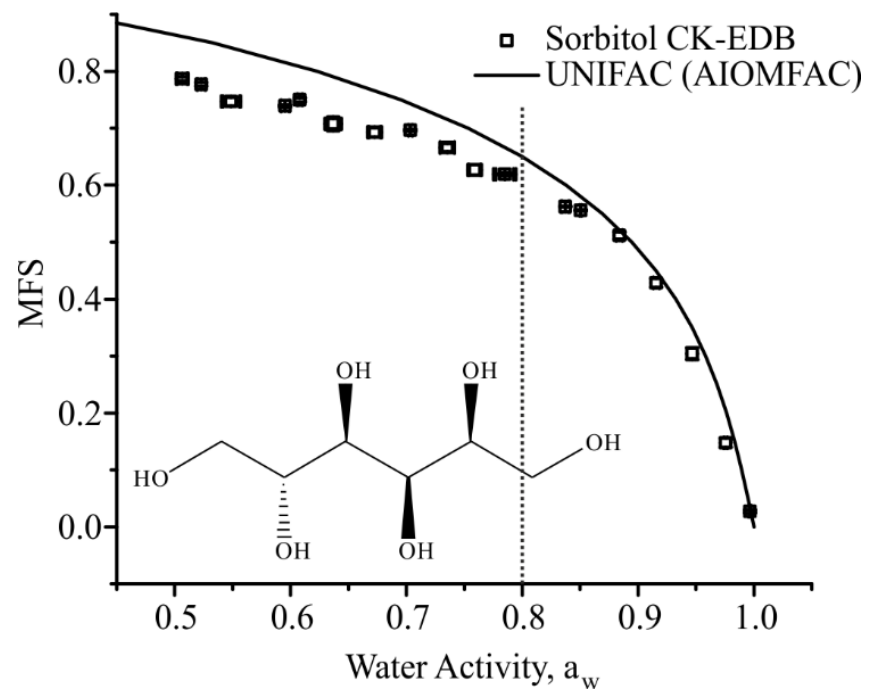

Table S29.1: Pure component refractive index $\left(n_{\text {melt }}\right)$ determined using molar refraction where the melt density ( $\left.\rho_{\text {melt }}\right)$ is determined using a polynomial fit of density to the square root of MFS (MFS $\left.{ }^{1 / 2}=\mathrm{x}\right)$. Bulk values used are available in Cai et al. (2016). Upper and lower refer to $95 \%$ confidence limits for fits to experimental data.

\begin{tabular}{c|cc|cccc}
\hline & & & \multicolumn{4}{|c}{ Polynomial fit $\left(\rho_{\text {sol }}=\mathrm{a}+\mathrm{b}_{1} \mathrm{x}+\mathrm{b}_{2} \mathrm{x}^{2}+\mathrm{b}_{3} \mathrm{x}^{3}\right)$} \\
& $n_{\text {melt }}$ & $\rho_{\text {melt }} / \mathrm{g} . \mathrm{cm}^{-3}$ & $\mathrm{a}$ & $\mathrm{b}_{1}$ & $\mathrm{~b}_{2}$ & $\mathrm{~b}_{3}$ \\
\hline \hline Best & 1.5244 & 1.4231 & 997.8 & 8.6 & 286.1 & 130.7 \\
\hline Upper & 1.5267 & 1.4333 & 997.8 & 24.74 & 234.56 & 175.54 \\
\hline Lower & 1.5220 & 1.4128 & 997.8 & -7.6 & 337.59 & 85.83 \\
\hline
\end{tabular}

Table S29.2: Tabulated experimental data points shown in Fig S29.1.

\begin{tabular}{ccc|cc}
\hline $\mathrm{a}_{\mathrm{w}}$ & error $\mathrm{a}_{\mathrm{w}}(+\mathrm{ve})$ & error $\mathrm{a}_{\mathrm{w}}(-\mathrm{ve})$ & MFS & error MFS \\
\hline \hline .50647 & 0.00432 & 0.00512 & 0.78667 & 0.00341 \\
0.52291 & 0.0031 & 0.00369 & 0.7771 & 0.00307 \\
0.54873 & 0.00705 & 0.00838 & 0.74672 & 0.00731 \\
0.59535 & 0.00322 & 0.00389 & 0.73916 & 0.00193 \\
0.60809 & 0.0019 & 0.0023 & 0.74976 & 0.00343 \\
0.63682 & 0.00605 & 0.00728 & 0.70773 & 0.01216 \\
0.67255 & 0.00497 & 0.00601 & 0.69271 & 0.00163 \\
0.7035 & 0.00148 & 0.00183 & 0.69648 & 0.00694 \\
0.73531 & 0.00619 & 0.00619 & 0.66608 & 0.00941 \\
0.75896 & 0.00493 & 0.00599 & 0.62673 & 0.00237 \\
0.78492 & 0.00775 & 0.00958 & 0.61901 & $9.55991 \mathrm{E}-4$ \\
0.83722 & 0.00384 & 0.0025 & 0.56241 & 0.00118 \\
0.85049 & $9.622 \mathrm{E}-4$ & $8.165 \mathrm{E}-4$ & 0.5556 & 0.00629 \\
0.88386 & 0.00262 & 0.0027 & 0.51154 & 0.0076 \\
0.91574 & 0.00253 & 0.00266 & 0.4286 & 0.01053 \\
0.94681 & 0.00245 & 0.00245 & 0.30429 & 0.00774 \\
0.97555 & 0.0014 & 0.00139 & 0.14769 & 0.00293 \\
0.99655 & 0.00112 & $6.78573 \mathrm{E}-4$ & 0.02751 & \\
\hline
\end{tabular}




\section{S30 D-(+)-Trehalose Dihydrate Hygroscopicity}

Fig S30.1: Hygroscopicity of D-(+)-trehalose dihydrate (Sigma Aldrich $\geq 99 \%$ ), at 293.15 K. Open squares, these experiments; solid line, UNIFAC model. Data taken at RHs lower than indicated by the dashed black line show increased error in hygroscopicity retrieval due to the imposition of a kinetic limitation on water transport.
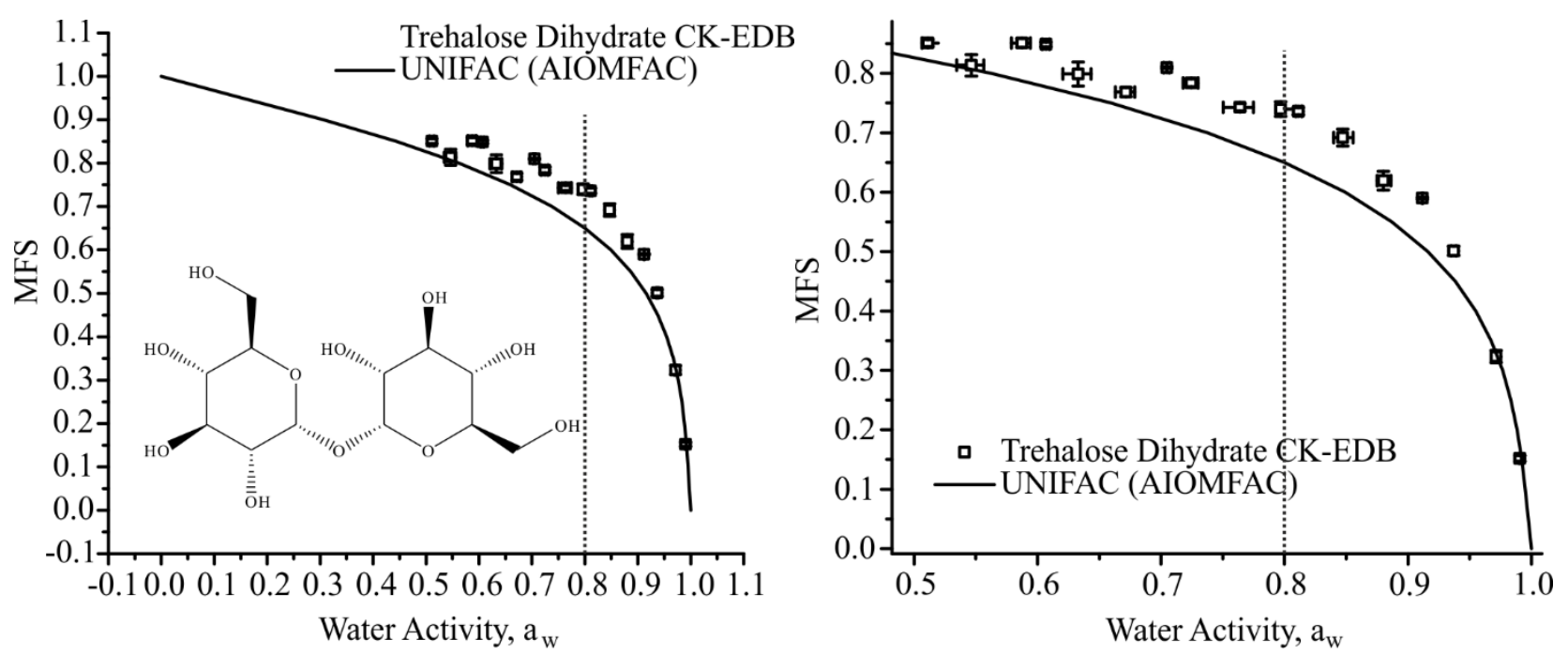

Table S30.1: Pure component refractive index $\left(n_{\text {melt }}\right)$ determined using molar refraction where the melt density $\left(\rho_{\text {melt }}\right)$ is determined using a polynomial fit of density to the square root of MFS $\left(\mathrm{MFS}^{1 / 2}=\mathrm{x}\right)$. Bulk values used are available in Cai et al. (2016). Upper and lower refer to $95 \%$ confidence limits for fits to experimental data.

\begin{tabular}{c|cc|cccc}
\hline & & & \multicolumn{4}{|c}{ Polynomial fit $\left(\rho_{\text {sol }}=\mathrm{a}+\mathrm{b}_{1} \mathrm{x}+\mathrm{b}_{2} \mathrm{x}^{2}+\mathrm{b}_{3} \mathrm{x}^{3}\right)$} \\
& $n_{\text {melt }}$ & $\rho_{\text {melt }} / \mathrm{g} . \mathrm{cm}^{-3}$ & $\mathrm{a}$ & $\mathrm{b}_{1}$ & $\mathrm{~b}_{2}$ & $\mathrm{~b}_{3}$ \\
\hline \hline Best & 1.5193 & 1.4682 & 997.8 & 8.2 & 284.3 & 177.8 \\
\hline Upper & 1.5211 & 1.4734 & 997.8 & 11.6 & 269.79 & 194.19 \\
\hline Lower & 1.5175 & 1.4629 & 997.8 & 4.87 & 298.84 & 161.43 \\
\hline
\end{tabular}

Table S30.2: Tabulated experimental data points shown in Fig S30.1.

\begin{tabular}{ccc|cc}
\hline $\mathrm{a}_{\mathrm{w}}$ & error $\mathrm{a}_{\mathrm{w}}(+\mathrm{ve})$ & error $\mathrm{a}_{\mathrm{w}}(-\mathrm{ve})$ & MFS & error MFS \\
\hline \hline .51123 & 0.00397 & 0.0047 & 0.8511 & 0.00561 \\
0.54636 & 0.01007 & 0.01196 & 0.81364 & 0.01816 \\
0.5873 & 0.007 & 0.00844 & 0.85121 & 0.00732 \\
0.60689 & 0.00263 & 0.00319 & 0.84879 & 0.00386 \\
0.63303 & 0.01031 & 0.01244 & 0.79889 & 0.02031 \\
0.67154 & 0.00716 & 0.00861 & 0.76858 & 0.00196 \\
0.70479 & 0.00212 & 0.00262 & 0.80977 & 0.00669 \\
0.72437 & 0.00577 & 0.00642 & 0.78413 & 0.00611 \\
0.76384 & 0.01102 & 0.01364 & 0.743 & 0.01219 \\
0.79679 & 0.00422 & 0.00225 & 0.7399 & 0.0059 \\
0.81122 & 0.00282 & 0.00195 & 0.73624 & 0.01427 \\
0.84712 & 0.00837 & 0.00721 & 0.69205 & 0.01589 \\
0.88007 & 0.00598 & 0.00498 & 0.61945 & 0.00159 \\
0.9118 & $5.25851 \mathrm{E}-4$ & $5.4066 \mathrm{E}-4$ & 0.58998 & 0.00792 \\
0.93698 & 0.00204 & 0.00204 & 0.50101 & 0.01015 \\
0.97142 & 0.00151 & 0.00149 & 0.3233 & 0.00476 \\
0.99054 & $4.05516 \mathrm{E}-4$ & $4.09208 \mathrm{E}-4$ & 0.15195 & \\
\hline
\end{tabular}




\section{S31. Galactose Hygroscopicity}

Fig S31.1: Hygroscopicity of (Sigma Aldrich $\geq 99 \%$ ), at 293.15 K. Open squares, these experiments; solid line, UNIFAC model. Data taken at RHs lower than indicated by the dashed black line show increased error in hygroscopicity retrieval due to the imposition of a kinetic limitation on water transport.
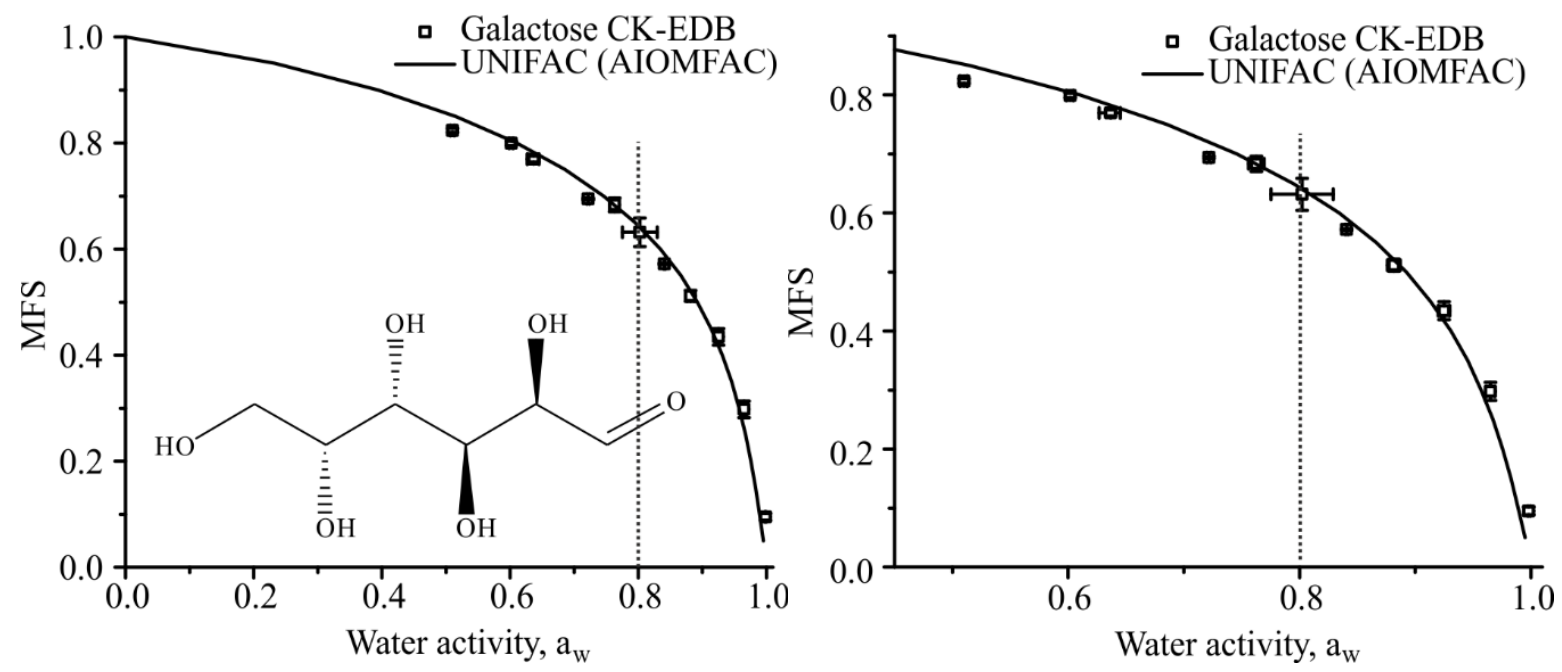

Table SI.31.1: Pure component refractive index $\left(n_{\text {melt }}\right)$ is determined using molar refraction, assuming ideal mixing for calculation of the melt density ( $\rho_{\text {melt }}$ ), from bulk data available in Cai et al. (2016). The variation of density as a function of the root of solute mass fraction $\left(\mathrm{MFS}^{1 / 2}=\mathrm{x}\right)$ is represented by polynomial fit parameters. Upper and lower refer to 95 $\%$ confidence limits for fits to experimental data, (Section 2.2 in manuscript).

\begin{tabular}{c|c|c|cccc}
\hline & & & \multicolumn{4}{|c}{ Polynomial fit $\left(\rho_{\text {sol }}=\mathrm{a}+\mathrm{b}_{1} \mathrm{x}+\mathrm{b}_{2} \mathrm{x}^{2}+\mathrm{b}_{3} \mathrm{x}^{3}\right)$} \\
& $n_{\text {melt }}$ & $\rho_{\text {melt }} / \mathrm{g} . \mathrm{cm}^{-3}$ & $\mathrm{a}$ & $\mathrm{b}_{1}$ & $\mathrm{~b}_{2}$ & $\mathrm{~b}_{3}$ \\
\hline \hline Best & 1.5885 & 1.6306 & 997.36 & 403.27 & 83.09 & 150.11 \\
\hline Upper & 1.5892 & 1.6351 & 996.67 & 165.3 & -284.07 & 752.22 \\
\hline Lower & 1.5878 & 1.6261 & 997.37 & 399.69 & 83.4 & 145.36 \\
\hline
\end{tabular}

Table S31.2: Tabulated experimental data points shown in Fig S31.1.

\begin{tabular}{ccc|cc}
\hline $\mathrm{a}_{\mathrm{w}}$ & error $\mathrm{a}_{\mathrm{w}}(+\mathrm{ve})$ & error $\mathrm{a}_{\mathrm{w}}(-\mathrm{ve})$ & MFS & error MFS \\
\hline \hline .50996 & 0.00287 & 0.0034 & 0.82372 & 0.00382 \\
0.60189 & 0.00267 & 0.00323 & 0.7993 & 0.00405 \\
0.63684 & 0.00839 & 0.01012 & 0.76963 & 0.0055 \\
0.72183 & 0.0016 & 0.00199 & 0.69438 & 0.00194 \\
0.76282 & 0.00662 & 0.00694 & 0.68348 & 0.01289 \\
0.80226 & 0.02704 & 0.02704 & 0.6317 & 0.02723 \\
0.84064 & 0.00138 & $8.91966 \mathrm{E}-4$ & 0.572 & 0.00141 \\
0.88152 & 0.00559 & 0.00561 & 0.51157 & 0.01025 \\
0.92485 & 0.00483 & 0.00491 & 0.43437 & 0.01532 \\
0.96504 & 0.00377 & 0.00374 & 0.29773 & 0.01536 \\
0.99822 & 0.00115 & $7.88489 \mathrm{E}-4$ & 0.09505 & 0.00656 \\
\end{tabular}




\section{S32 Xylose Hygroscopicity}

Fig S32.1: Hygroscopicity of (Sigma Aldrich $\geq 99 \%$ ), at 293.15 K. Open squares, these experiments; solid line, UNIFAC model.
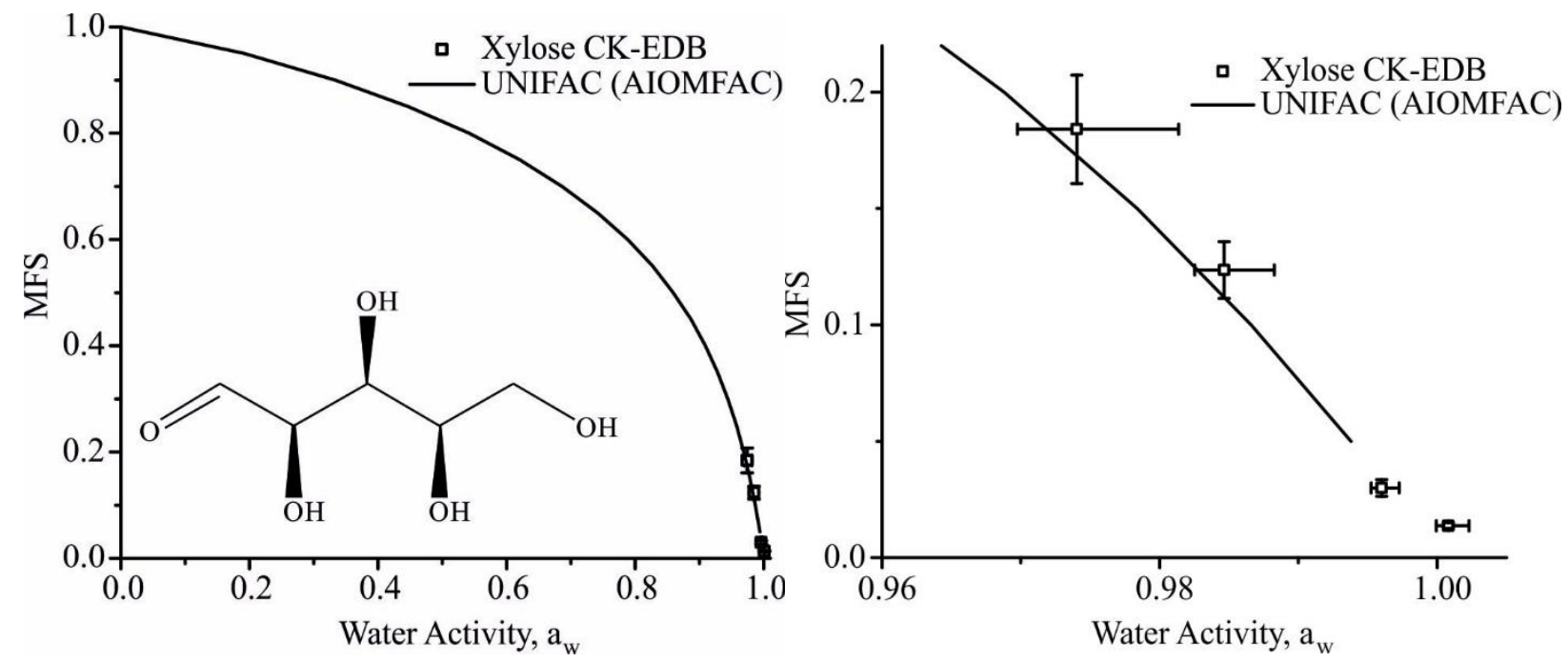

Table S32.1: Pure component refractive index $\left(n_{\text {melt }}\right)$ is determined using molar refraction, assuming ideal mixing for calculation of the melt density ( $\left.\rho_{\text {melt }}\right)$, from bulk data available in Cai et al. (2016). The variation of density as a function of the root of solute mass fraction $\left(\mathrm{MFS}^{1 / 2}=\mathrm{x}\right.$ ) is represented by polynomial fit parameters. Upper and lower refer to 95 $\%$ confidence limits for fits to experimental data, (Section 2.2 in manuscript).

\begin{tabular}{c|cc|cccc}
\hline & & & \multicolumn{4}{|c}{ Polynomial fit $\left(\rho_{\text {sol }}=\mathrm{a}+\mathrm{b}_{1} \mathrm{x}+\mathrm{b}_{2} \mathrm{x}^{2}+\mathrm{b}_{3} \mathrm{x}^{3}\right)$} \\
& $n_{\text {melt }}$ & $\rho_{\text {melt }} /{\mathrm{g} . \mathrm{cm}^{-3}}$ & $\mathrm{a}$ & $\mathrm{b}_{1}$ & $\mathrm{~b}_{2}$ & $\mathrm{~b}_{3}$ \\
\hline \hline Best & 1.5615 & 1.5626 & 996.73 & 127.69 & -163.53 & 597.09 \\
\hline Upper & 1.5619 & 1.5653 & 996.74 & 126.37 & -159.45 & 591.57 \\
\hline Lower & 1.5611 & 1.5598 & 996.72 & 128.97 & -167.5 & 602.42 \\
\hline
\end{tabular}

Table S32.2: Tabulated experimental data points shown in Fig S32.1.

\begin{tabular}{ccc|cc}
\hline $\mathrm{a}_{\mathrm{w}}$ & error $\mathrm{a}_{\mathrm{w}}(+\mathrm{ve})$ & error $\mathrm{a}_{\mathrm{w}}(-\mathrm{ve})$ & MFS & error MFS \\
\hline \hline 0.97404 & 0.00732 & 0.00429 & 0.1841 & 0.0233 \\
0.98465 & 0.00361 & 0.00212 & 0.12356 & 0.01215 \\
0.996 & 0.00127 & $7.43479 \mathrm{E}-4$ & 0.02995 & 0.00361 \\
1.00081 & 0.00148 & $8.71845 \mathrm{E}-4$ & 0.01372 & 0.0012 \\
& & & & \\
\hline
\end{tabular}




\section{S33 2,3-Dimethyl Succinic Acid Hygroscopicity}

Fig S33.1: Hygroscopicity of 2,3-dimethyl succinic acid (Sigma Aldrich $\geq 99 \%$ ), at 293.15 K. Open squares, these experiments; solid line, UNIFAC model. (Density treatment for 2,2-dimethyl succinic acid used.)

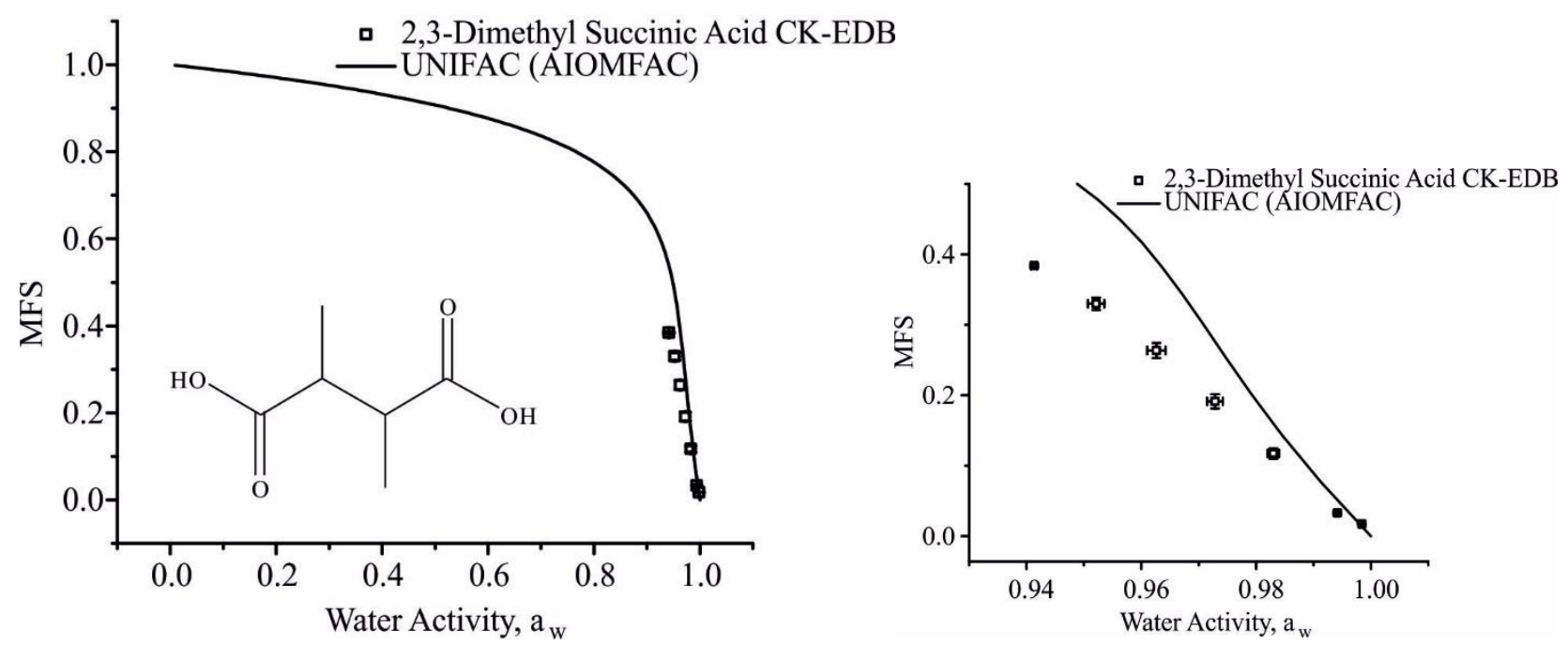

Table S33.2: Tabulated experimental data points shown in Fig S33.1.

\begin{tabular}{ccc|cc}
\hline $\mathrm{a}_{\mathrm{w}}$ & error $\mathrm{a}_{\mathrm{w}}(+\mathrm{ve})$ & error $\mathrm{a}_{\mathrm{w}}(-\mathrm{ve})$ & MFS & error MFS \\
\hline \hline 0.94132 & $5.11673 \mathrm{E}-4$ & $5.12405 \mathrm{E}-4$ & 0.38395 & 0.00207 \\
0.95214 & 0.00144 & 0.00144 & 0.32979 & 0.00859 \\
0.96262 & 0.00159 & 0.00159 & 0.26369 & 0.01065 \\
0.97285 & 0.00138 & 0.00138 & 0.19135 & 0.01011 \\
0.98303 & 0.001 & 0.001 & 0.11733 & 0.00731 \\
0.99417 & $2.09751 \mathrm{E}-4$ & $2.24291 \mathrm{E}-4$ & 0.03301 & 0.00121 \\
0.99844 & $2.59195 \mathrm{E}-4$ & $4.09162 \mathrm{E}-4$ & 0.01724 & $4.61378 \mathrm{E}-4$ \\
& & & & \\
\hline
\end{tabular}




\section{S34 Dimethyl Malonic Acid Hygroscopicity}

Figure S34.1: Hygroscopicity of (Sigma Aldrich $98 \%$ ), at 293.15 K. Open squares, these experiments; solid line, UNIFAC model. (Density treatment for methyl succinic acid used.)
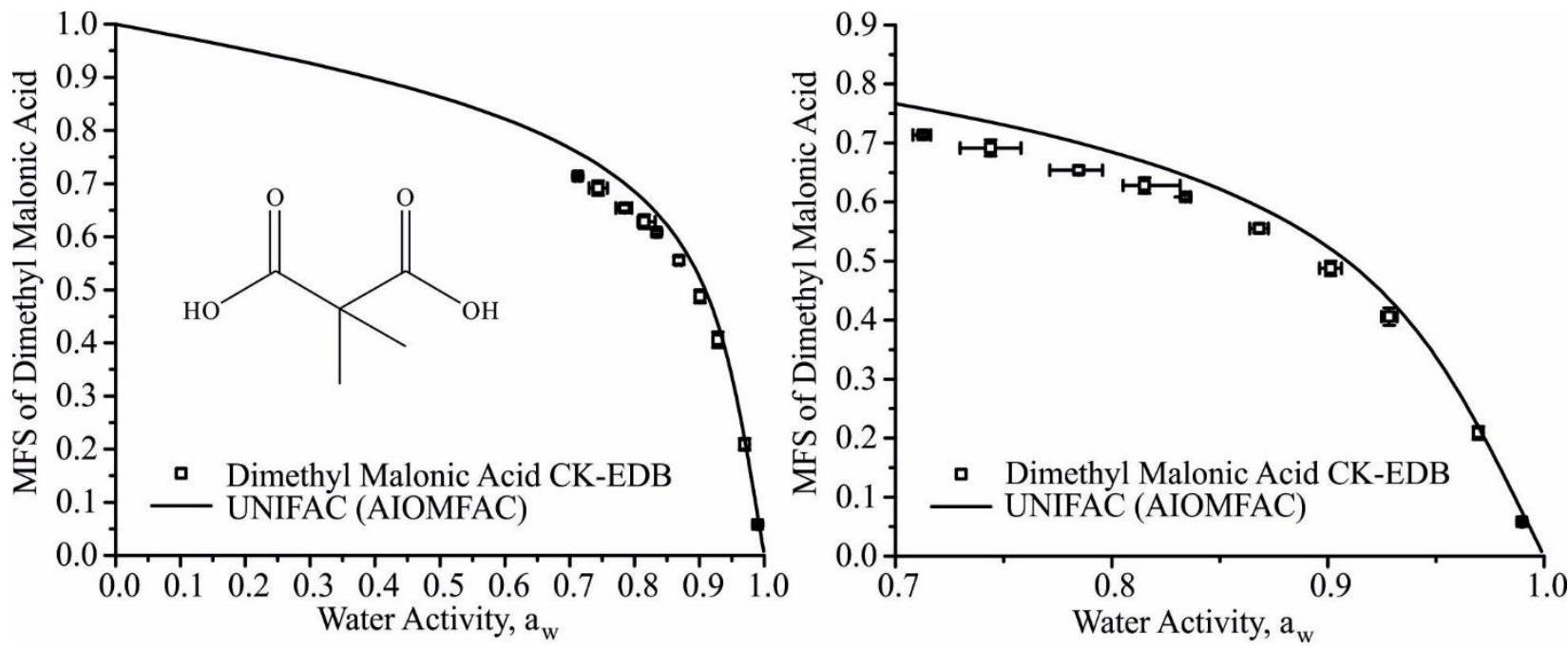

Table S34.2: Tabulated experimental data points shown in Figure S34.1.

\begin{tabular}{ccc|cc}
\hline $\mathrm{a}_{\mathrm{w}}$ & error $\mathrm{a}_{\mathrm{w}}(+\mathrm{ve})$ & error $\mathrm{a}_{\mathrm{w}}(-\mathrm{ve})$ & MFS & error MFS \\
\hline \hline 0.71262 & 0.00362 & 0.00449 & 0.7136 & 0.00301 \\
0.744 & 0.0141 & 0.0141 & 0.69155 & 0.01343 \\
0.78481 & 0.01088 & 0.01348 & 0.65412 & 0.00614 \\
0.81516 & 0.01647 & 0.00985 & 0.62813 & 0.01311 \\
0.83412 & 0.00246 & 0.00229 & 0.60844 & 0.00357 \\
0.86818 & 0.00422 & 0.00426 & 0.5554 & 0.00729 \\
0.90119 & 0.00509 & 0.00506 & 0.48761 & 0.01203 \\
0.92833 & 0.00366 & 0.00365 & 0.40593 & 0.01475 \\
0.96965 & 0.00157 & 0.00194 & 0.2089 & 0.01089 \\
0.9897 & $4.75033 \mathrm{E}-4$ & $4.76981 \mathrm{E}-4$ & 0.05824 & 0.00271 \\
\end{tabular}




\section{S35 Aspartic Acid Hygroscopicity}

Fig S35.1: Hygroscopicity of aspartic acid (Sigma Aldrich $\geq 99 \%$ ), at 293.15 K. Open squares, these experiments; solid line, UNIFAC model. (Density treatment for alanine used)

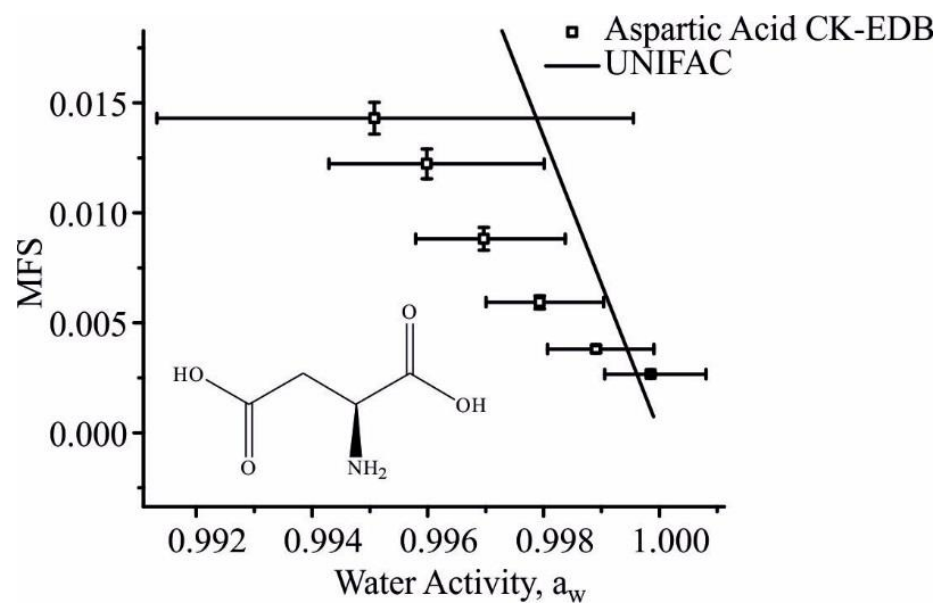

Table S35.1: Tabulated experimental data points shown in Fig S35.1.

\begin{tabular}{ccc|cc}
\hline $\mathrm{a}_{\mathrm{w}}$ & error $\mathrm{a}_{\mathrm{w}}(+\mathrm{ve})$ & error $\mathrm{a}_{\mathrm{w}}(-\mathrm{ve})$ & MFS & error MFS \\
\hline \hline 0.99507 & 0.00448 & 0.00375 & 0.01431 & $7.18 \mathrm{E}-04$ \\
0.99599 & 0.00202 & 0.0017 & 0.01223 & $6.83 \mathrm{E}-04$ \\
0.99697 & 0.00141 & 0.00118 & 0.00882 & $5.15 \mathrm{E}-04$ \\
0.99793 & 0.00111 & $9.28 \mathrm{E}-04$ & 0.00594 & $3.01 \mathrm{E}-04$ \\
0.99891 & 0.001 & $8.39 \mathrm{E}-04$ & 0.00381 & $1.64 \mathrm{E}-04$ \\
0.99985 & $9.52 \mathrm{E}-04$ & $7.98 \mathrm{E}-04$ & 0.00266 & $8.72 \mathrm{E}-05$ \\
\end{tabular}




\section{S36 Asparagine Hygroscopicity}

Fig S36.1: Hygroscopicity of asparagine (Sigma Aldrich $\geq 98 \%$ ), at 293.15 K. Open squares, these experiments; solid line, UNIFAC model. (Density treatment for alanine used)
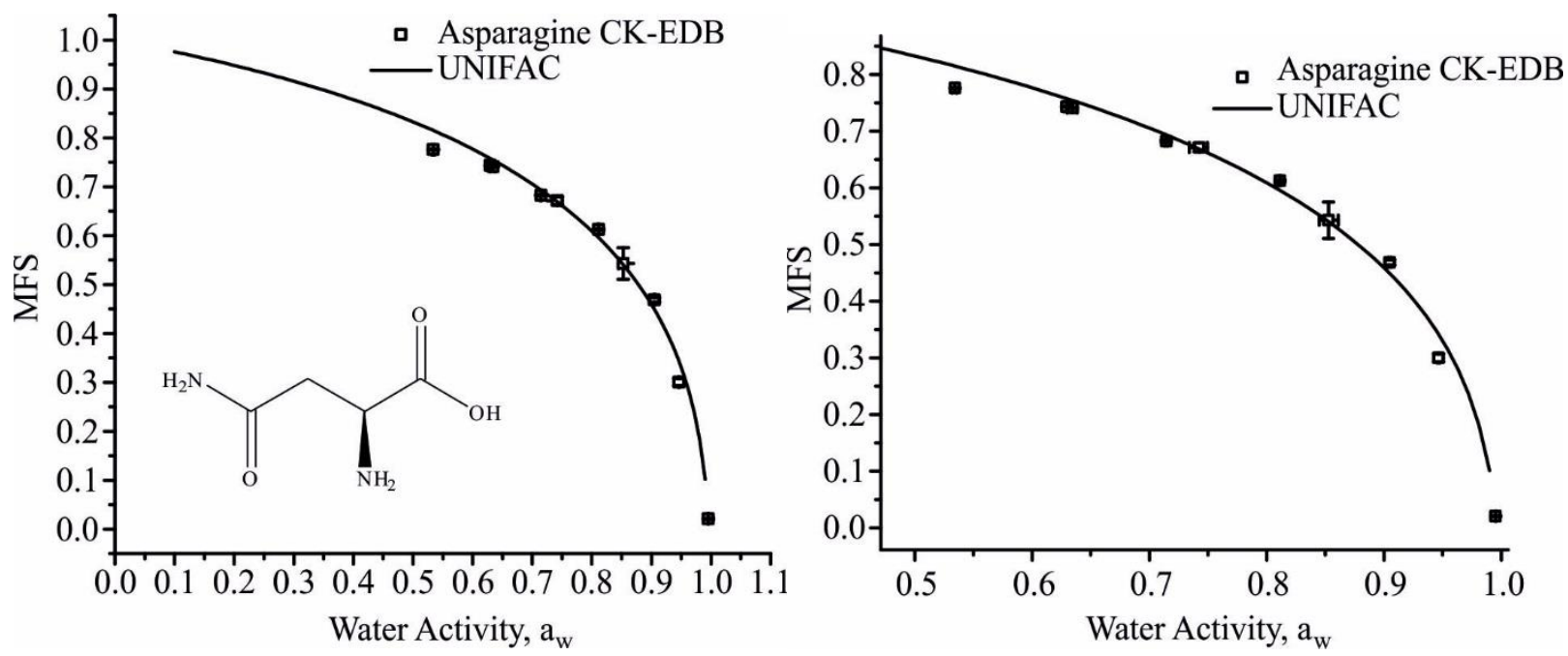

Table S36.1: Tabulated experimental data points shown in Figure S36.1.

\begin{tabular}{ccc|cc}
\hline $\mathrm{a}_{\mathrm{w}}$ & error $\mathrm{a}_{\mathrm{w}}(+\mathrm{ve})$ & error $\mathrm{a}_{\mathrm{w}}(-\mathrm{ve})$ & MFS & error MFS \\
\hline \hline 0.53409 & 0.00178 & 0.00213 & 0.77577 & 0.00129 \\
0.62935 & 0.00189 & 0.0023 & 0.74326 & 0.00101 \\
0.63444 & 0.00381 & 0.00465 & 0.74081 & 0.00101 \\
0.71441 & 0.00113 & 0.0014 & 0.68254 & 0.00175 \\
0.74237 & 0.007 & 0.00854 & 0.67146 & 0.00782 \\
0.81123 & $8.45796 \mathrm{E}-4$ & $8.49613 \mathrm{E}-4$ & 0.61254 & 0.00185 \\
0.85278 & 0.00812 & 0.00813 & 0.54286 & 0.03203 \\
0.9048 & 0.00102 & $9.46055 \mathrm{E}-4$ & 0.46853 & 0.00454 \\
0.94641 & 0.00108 & 0.0011 & 0.3002 & 0.00124 \\
0.9951 & $2.80427 \mathrm{E}-4$ & $2.96722 \mathrm{E}-4$ & 0.02083 & \\
\hline
\end{tabular}

S37 Errors in Density and Refractive Index Parametrisations and their Impact on Hygroscopicity

Fig S37.1 Parametrisation for (a) density based on ideal mixing and bulk measured values for density up to the solubility limit and (b) refractive index predicted beyond the solubility limit using molar refraction. In both (a) and (b) dashed lines indicate the uncertainty envelope in the parametrisations. All bulk experimental values of aqueous density and refractive index are available in the supplementary information of Cai et al. (2016). In (c) measured equilibrium hygroscopicity curves are presented with upper and lower error envelope arising from the uncertainties in density and refractive index which is too small to be obvious. 


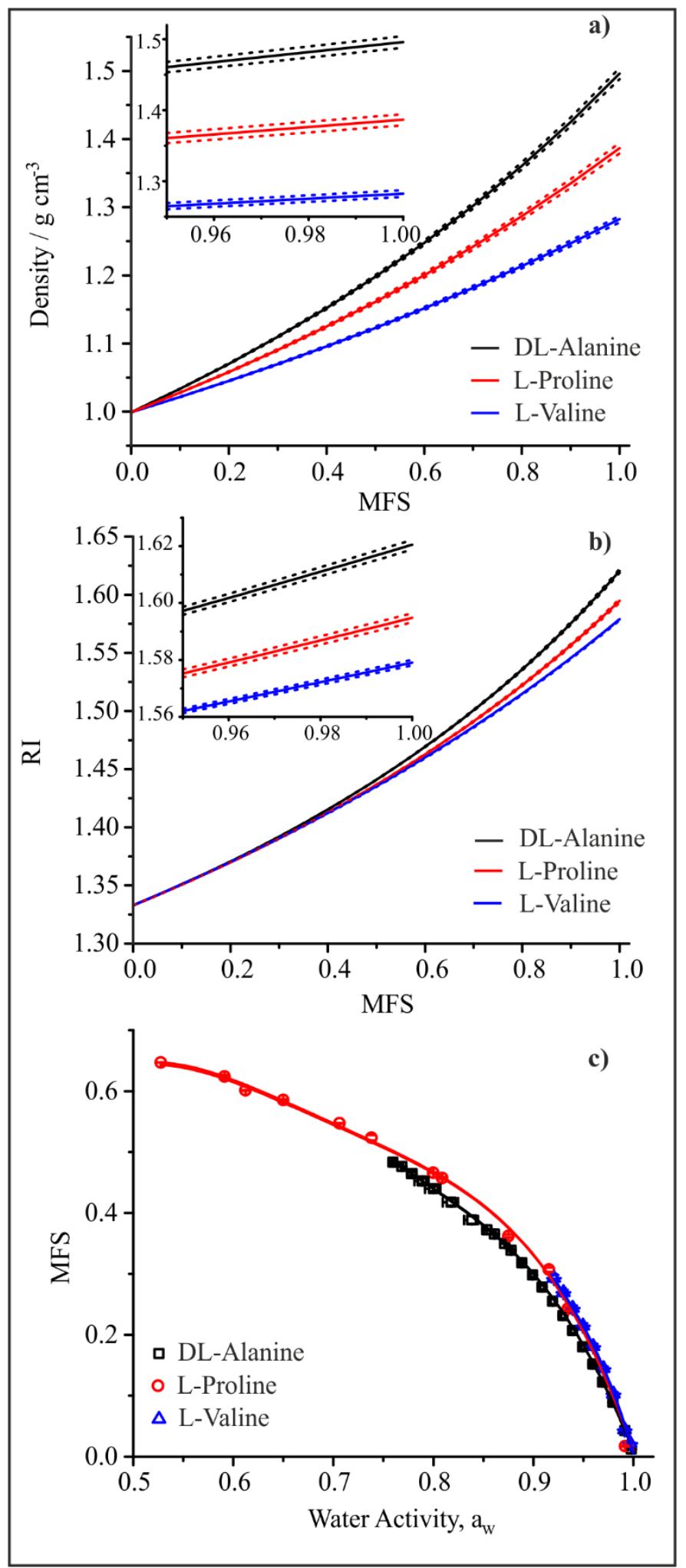


Fig S38.1 The difference in mass fraction of solute ( $\triangle$ MFS) between values predicted by UNIFAC and experimental values (a) oxalic acid, (b) malonic acid, (c) succinic acid and (d) glutaric acid.

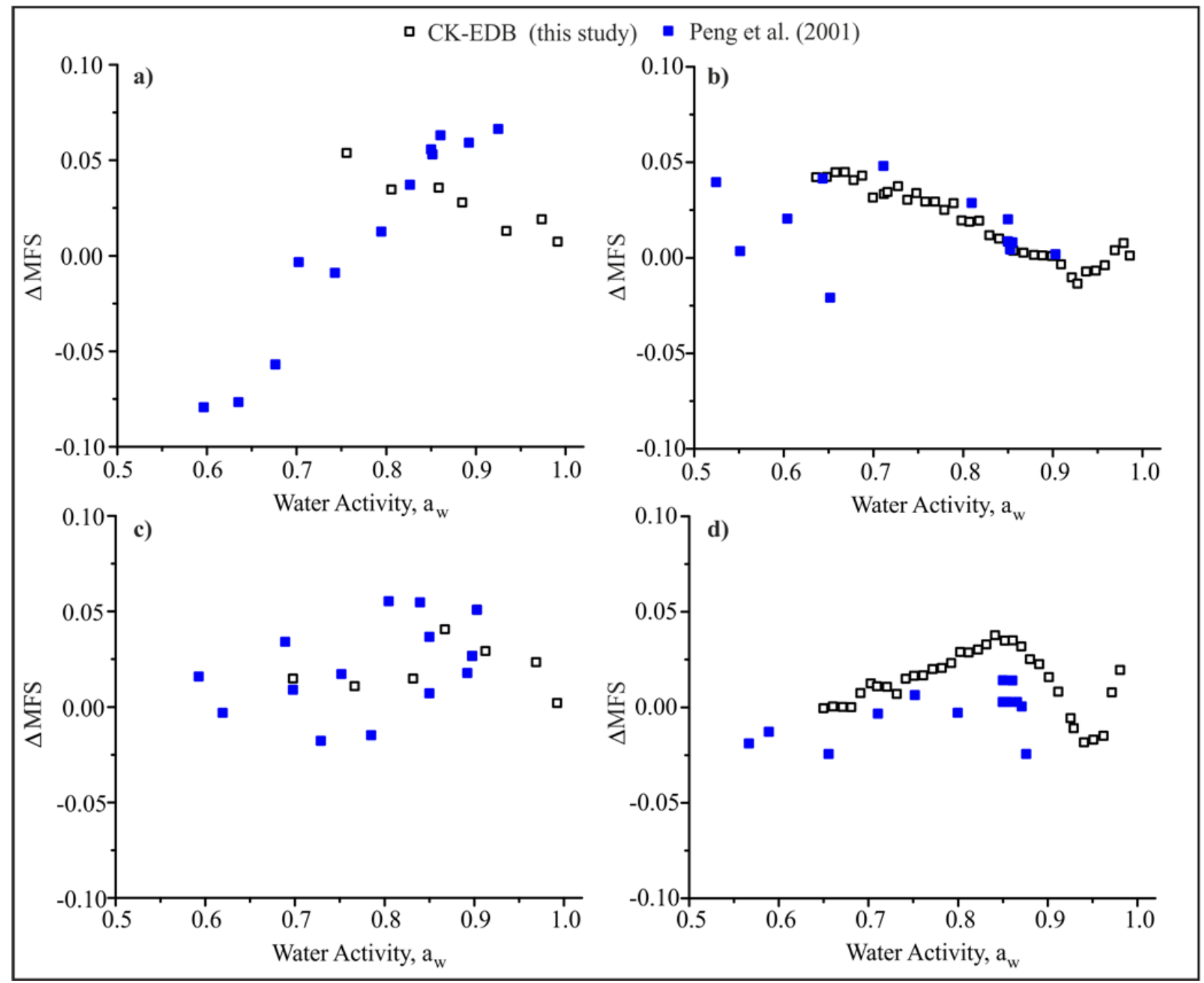

\section{S39 Viscosity, Diffusion Constant and Timescale of Diffusional Mixing}

The kinetic modelling framework used in the analysis of the droplet evaporation events is valid only in the absence of a bulk-kinetic limitation on near surface composition, i.e. the particle must be assumed to be homogeneous in composition. Such a limitation was obvious for hygroscopicity measurements of trehalose, galactose and sorbitol at RH's lower than $80 \%$. To ensure the measurements are not compromised by bulk diffusion, we consider two important factors.

Firstly, the impact of viscosity on the hygroscopicity retrievals becomes very obvious when we consider the consistency and uncertainty in the raw hygroscopic growth curves determined from different droplets evaporating into differing RHs. Droplets drying into different RHs reach different compositions at different times, and will retain different amounts of water because of different drying rates. This leads to an artificially low MFS at a particular RH which then slowly returns to the equilibrium curve overtime. Thus, an inconsistency is apparent between retrieved hygroscopic growth curves (or MFS vs $a_{w}$ ) when drying into different RHs. An example of this is shown in Figure S39.1, where we report unbinned hygroscopicity data for alanine (a non-viscous amino acid) and trehalose (viscous at RHs lower than 80\%). It is clear here that the different portions of the hygroscopic curves retrieved from measurements at different $\mathrm{RHs}$ are consistent for alanine but not for trehalose. A further easy way to identify this retention of water in a particle that is not fully 
equilibrated is simply to measure the much longer time-dependence in size once the initial evaporation of water has stopped. In droplets that have reached a bulk diffusion limitation, the existence of a kinetic limitation is apparent in a steadily decreasing size as water continues to leave over a timescale longer than $10 \mathrm{~s}$.

Fig S39.1 a) Unbinned hygroscopicity data for the compound alanine. b) Unbinned hygroscopicity data for the compound trehalose. At $50 \%$ RH trehalose has a viscosity of 3.8 x 10 5 Pa.s (Song et al. 2016).

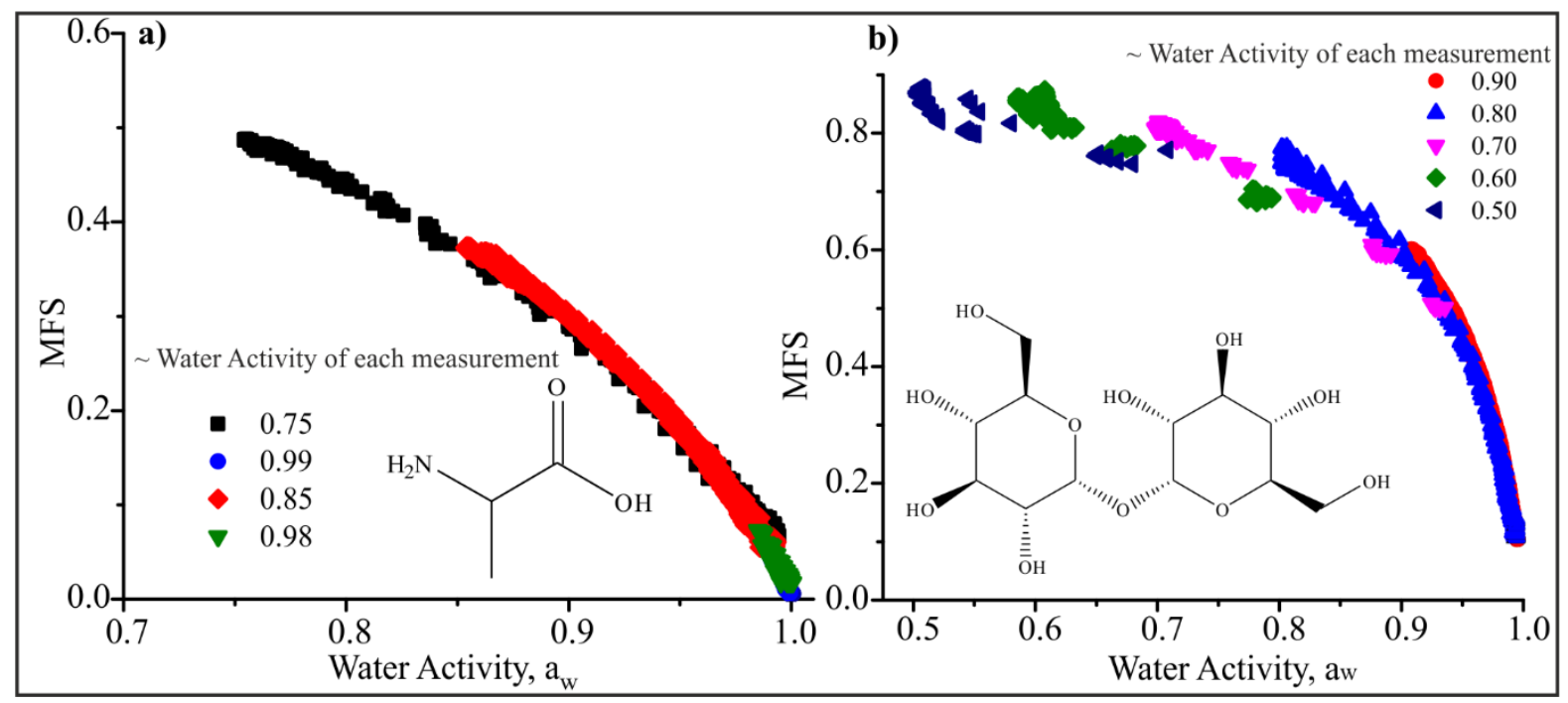

Secondly, we can determine the expected conditions under which we might expect problems to arise in retrieving hygroscopic growth curves from an evaporation measurement. Considering again trehalose at $80 \%$ $\mathrm{RH}$, an aqueous-trehalose droplet has a viscosity of $0.5 \mathrm{~Pa}$.s, increasing to $3.8 \times 10^{5} \mathrm{~Pa}$.s at $50 \% \mathrm{RH}$ (Song et al. 2016). Therefore, as the RH of the gas phase for the evaporation measurement is lowered, we can expect the increasing viscosity/decreasing diffusivity to become increasingly important. By contrast, for aqueouscarboxylic acid droplets, the viscosity never gets above $1 \mathrm{~Pa}$ s even at the driest RHs considered here (Song et al. 2016).

With these known dependencies of viscosity on water activity, we can estimate the timescale for diffusional mixing within a droplet, assuming that this provides an estimate of the timescale for an evaporating droplet to form a homogeneous mixture. This timescale must be considerably shorter than the evaporation timescale for our hygroscopicity estimations to be valid. First, the Stokes-Einstein equation is used to estimate the diffusion constant of water at varying viscosity (varying $\mathrm{RH}$ ).

$$
D=\frac{k_{B} T}{6 \pi r_{m o l} \eta}
$$

$D$ is the diffusion constant, $k_{B}$ is the Boltzmann constant, $T$ is temperature, $r_{m o l}$ is the molecular radius of water (taken as $1.375 \AA$ ) and $\eta$ is the viscosity. It should be noted that equation (1.1) is likely to provide a significant underestimate of the diffusion constant due to the failure of the Stokes-Einstein equation. At a viscosity of $100 \mathrm{~Pa} \mathrm{~s}$, the diffusion constant for water in sucrose is already more than one order of magnitude larger than estimated from the viscosity (Power et al. 2013). However, using diffusion constants estimated from (1.1) will provide an upper limit on the diffusional mixing timescale. The timescale for diffusional mixing, $\tau$, is then estimated using the expression

$$
\tau=\frac{a^{2}}{\pi^{2} D}
$$

where $a$ is the droplet radius (set as 10 microns in this calculation).

We compare the diffusional mixing timescales for aqueous droplets of trehalose, $\mathrm{NaCl}, \mathrm{NaNO}_{3}$ and glutaric acid in the newly added supplemental Figure S39.2 (and repeated below). Given that we have been able to report accurate hygroscopic growth curves for $\mathrm{NaNO}_{3}$ down to $50 \% \mathrm{RH}$ (see Rovelli et al. 2016 and the 
response to referee 2), it is clear that a final viscosity at $50 \%$ of $\sim 0.1$ Pa.s (Baldelli et al.) is insufficient to impede accurate measurement of the hygroscopicity. Indeed, this suggests that water transport in any aerosol droplet that maintains a viscosity lower than 0.1 Pa.s during drying should remain sufficiently fast to avoid a bulk diffusion limitation, permitting accurate hygrosocpicity measurements. As an example of the diacarboyxlic acids considered in this study, glutaric acid has a considerably lower viscosity at $50 \%$ RH of 0.01 Pa.s (Song et al. 2016), indicative of what we might expect for all such similar systems. By contrast, aqueous-trehalose droplets cross the $0.1 \mathrm{~Pa}$.s viscosity threshold at a water activity of $\sim 0.85$ (Song et al. 2016), commensurate with the deviation and increased scatter in the hygroscopicity measurements reported above for this compound.

Based on the two considerations above and to indicate clearly the water activity ranges over which we consider the hygroscopicity measurements to be valid for trehalose (S30), galactose (S31) and sorbitol (S29), we have added a dashed line to indicate where the data appear to become kinetically limited. We have added the following words to the captions of these Figures: "Data taken at RHs lower than indicated by the dashed black line show increased error in hygroscopicity retrieval due to the imposition of a kinetic limitation on water transport."

Fig S39.2 a) Viscosity of Trehalose, NaCl, NaNO3 and Glutaric Acid as a function of RH. b) Estimated diffusion constant as a function of RH. c) Timescale for diffusional mixing at the RH shown on $x$-axis. Dashed green line represents 1 second timescale for diffusional mixing.

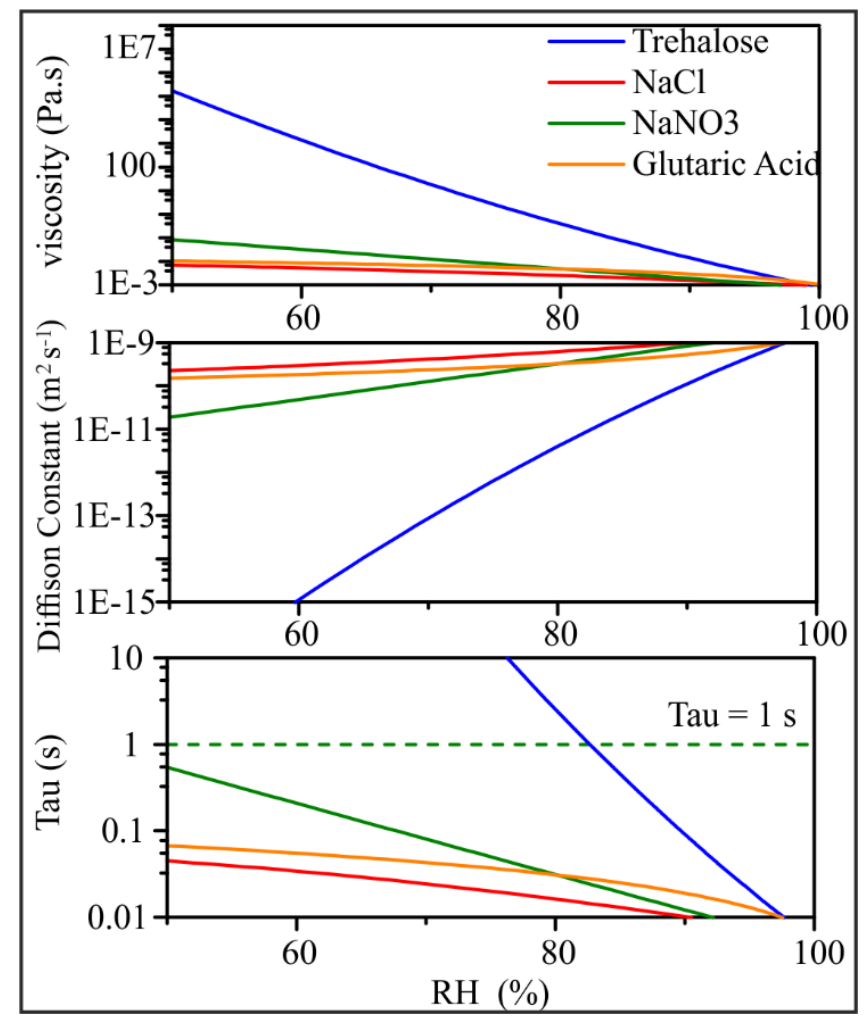

A. Baldelli, R. M. Power, R. E. H. Miles, J. P. Reid and R. Vehring Effect of crystallization kinetics on the properties of spray dried microparticles, Aerosol Science and Technology, 2016, 50:7, 693-704, DOI:10.1080/02786826.2016.1177163

R. M. Power, S. H. Simpson, J. P. Reid and A. J. Hudson, The transition from liquid to solid-like behaviour in ultrahigh viscosity aerosol particles, Chemical Science, 2013, 4, 2597, DOI: 10.1039/c3sc50682g

Y. Chul Song, A. E. Haddrell, B. R. Bzdek, J. P. Reid, T. Bannan, D. O. Topping,, C. Percival, and C. Cai Measurements and Predictions of Binary Component Aerosol Particle Viscosity J. Phys. Chem. A 2016, 120, 8123-8137, DOI: 10.1021/acs.jpca.6b07835 
S40 Differences between Cyclic and Open Chain Sugar Conformer Thermodynamic Predictions

Table S40.0: Table of UNIFAC groups for cyclic and open chain galactose and xylose.

\begin{tabular}{|c|c|c|}
\hline Compound & Open Chain (In Manuscript) & Cyclic \\
\hline $\begin{array}{l}\text { Galactose } \\
\text { Xylose }\end{array}$ & $\begin{array}{l}\mathrm{CHO}\left(\mathrm{CH}_{1}{ }^{(\mathrm{OH})}\right)_{4} \mathrm{CH}_{2}{ }^{(\text {alc })}(\mathrm{OH})_{5} \\
\left(\mathrm{CH}_{2}(\mathrm{OH})\right)_{3} \mathrm{CH}_{2}{ }^{(\text {alc })} \mathrm{CHO}(\mathrm{OH})_{4}\end{array}$ & $\begin{array}{l}\left(\mathrm{CH}^{[\mathrm{alc}]}\right)_{4}\left(\mathrm{CH}_{2}^{[\mathrm{OH}]}\right)\left(\mathrm{CHO}^{[\mathrm{ether}]}\right)(\mathrm{OH})_{4} \\
\left(\mathrm{CH}^{[\mathrm{OH}]}\right)_{4}\left(\mathrm{CHO}^{[\mathrm{ether}]}\right)(\mathrm{OH})_{4}\end{array}$ \\
\hline
\end{tabular}

Figure S40.0 Galactose and Xylose CK-EDB data as a function of MFS and water activity compared with predictions for both cyclic and open chain UNIFAC group thermodynamic predictions

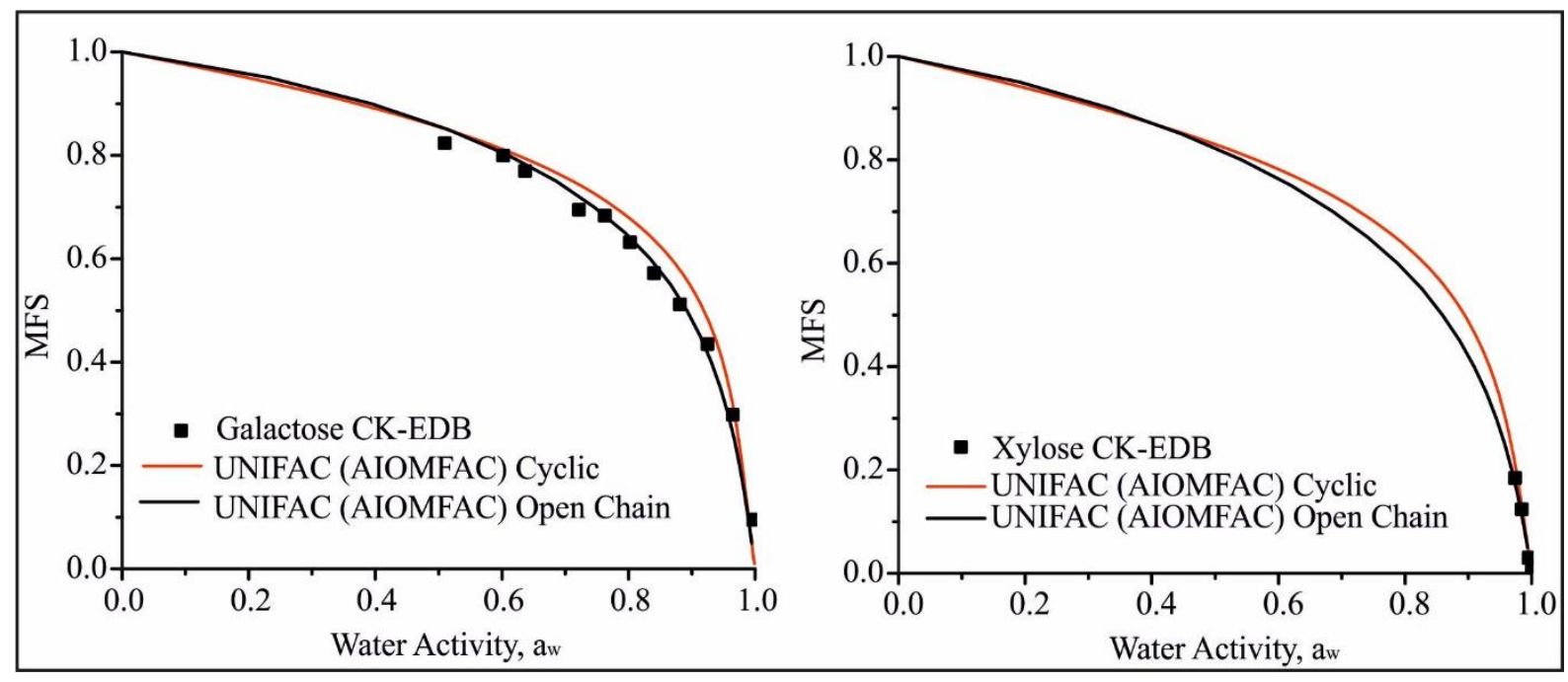

